\title{
Mesenchymal Stem Cells in Cartilage Repair
}

\section{Studies of Chondrogenesis In Vitro and In Vivo}

\author{
Doctoral Thesis
}

Rune Bruhn Jakobsen MD

2014

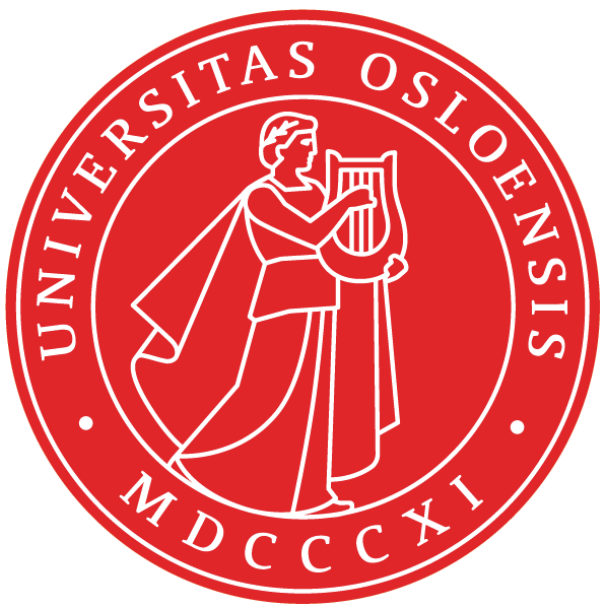

UNIVERSITY OF OSLO

\section{Faculty of Medicine}

Institute of Basic Medical Sciences, Department of Biochemistry

University of Oslo

and

Norwegian Center for Stem Cell Research and Department of Immunology Oslo University Hospital and University of Oslo 
(C) Rune Bruhn Jakobsen, 2014

Series of dissertations submitted to the Faculty of Medicine, University of Oslo No. 1786

ISBN 978-82-8264-814-1

All rights reserved. No part of this publication may be reproduced or transmitted, in any form or by any means, without permission.

Cover: Inger Sandved Anfinsen.

Printed in Norway: AIT Oslo AS.

Produced in co-operation with Akademika Publishing.

The thesis is produced by Akademika Publishing merely in connection with the thesis defence. Kindly direct all inquiries regarding the thesis to the copyright holder or the unit which grants the doctorate. 


\section{Table of Contents}

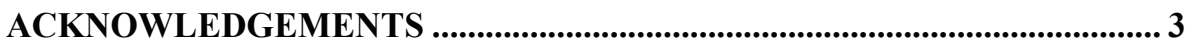

LIST OF PAPERS INCLUDED .................................................................... 6

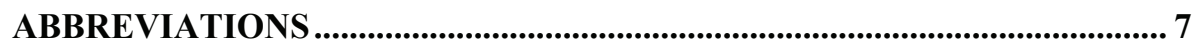

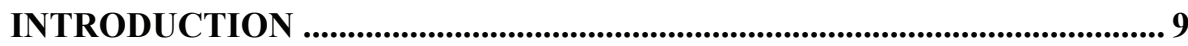

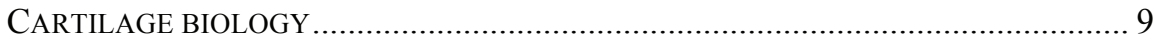

Cartilage embryology, anatomy, and physiology …………………………………....

IN VIVO CHONDROGENESIS AND ENDOCHONDRAL OSSIFICATION .......................... 14

In vitro de- and redifferentiation of chondrocytes.............................................................. 18

Extracellular matrix.................................................................................................. 19

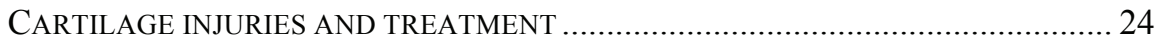

Grading and scoring systems for cartilage injury .......................................................... 24

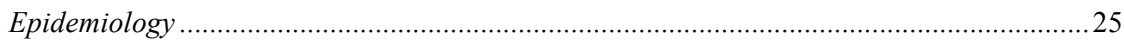

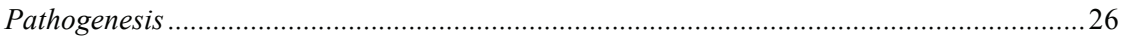

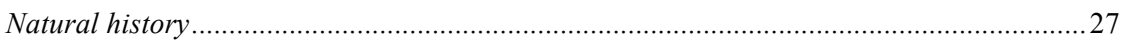

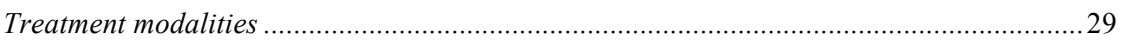

STEM CELL BIOLOGY ................................................................................. 36

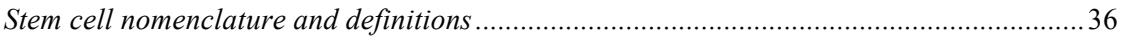

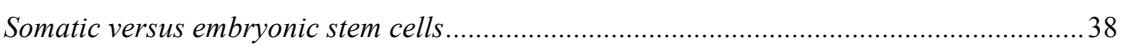

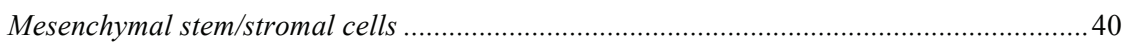

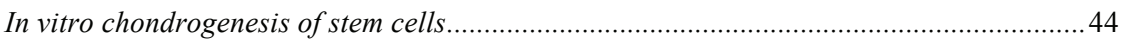

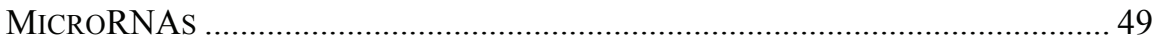

Role of microRNAs in cartilage biology and pathology ...................................................50

Future applications of microRNA in treatment, diagnosis, and prognosis ...........................52

AIMS OF THE STUDY .......................................................................................... 54

SUMMARY OF RESULTS ..................................................................................55

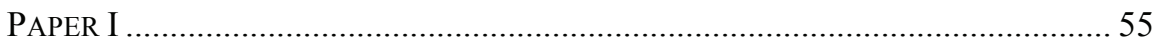

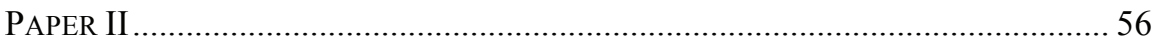

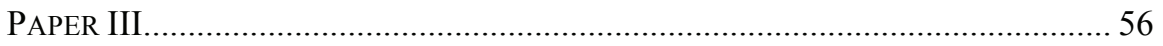

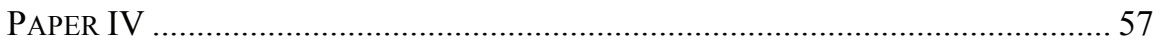

METHODOLOGICAL CONSIDERATIONS..................................................59

CELL CULTURE AND SUPPLEMENTS ........................................................... 59 
THREE-DIMENSIONAL CULTURE AND CHOICE OF SCAFFOLD.............................6 60

TRANSFECTIONS AND TRANSDUCTIONS ......................................................... 62

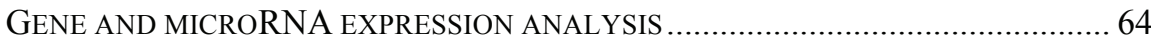

FUNCTIONAL ASSAYS (PROTEIN AND PROTEOGLYCAN QUANTIFICATION).......... 66

MICRORNA LUCIFERASE REPORTER ASSAY ................................................... 68

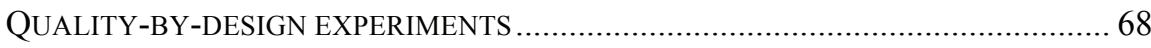

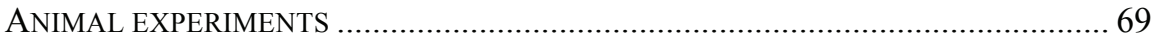

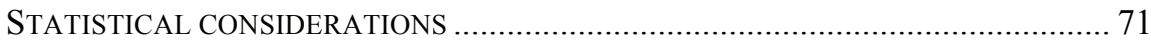

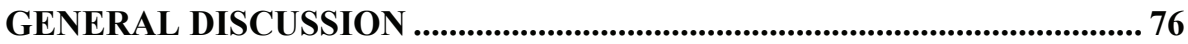

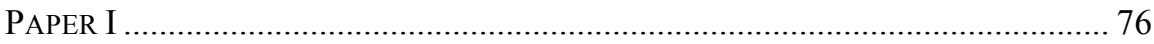

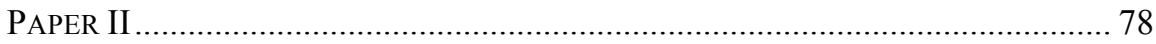

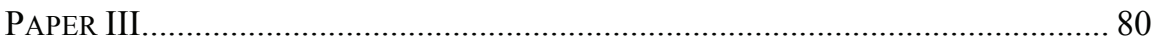

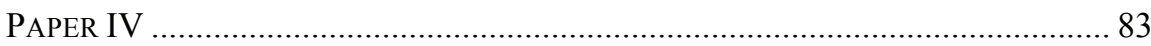

CONCLUSIONS AND FUTURE STUDIES .................................................. 85

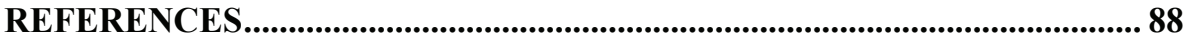




\section{Acknowledgements}

The present work was begun while I was a medical student in the research programme at the Faculty of Medicine at the University of Oslo from 2004 to 2007, and finalised after I returned to the laboratory in 2010. During this time, I have had the privilege of working with a large number of skilled and talented people whom I would like to acknowledge here:

First, I would like to thank my supervisor Jan E. Brinchmann. From the first time I set foot in the lab, Jan has been a constant optimistic and encouraging supervisor. His vast experience in the field of stem cells and genuine interest both in science and people created (and maintains) a unique and friendly environment which I am very happy and proud to be part of - and because of this I stayed for much longer than I anticipated almost a decade ago.

I am also very grateful to Lars Engebretsen who invited me in to the multifaceted research at Oslo Sports Trauma Research Center as a medical student and first introduced me to the clinical conundrum of cartilage repair. Lars juggles what seems to be a handful of full-time jobs, yet is and has been accessible for any question at any time with an astonishingly short response time, which I doubt anyone matches.

There are a number of people who are or have been in the lab or in other ways involved in the work presented here, whom I particularly wish to thank:

Tommy A. Karlsen for great teamwork and a huge effort in the lab, and for many and varied conversations about science, evolution, and our families along the way. Aboulghassem Shahdadfar for carefully teaching me how to behave in the lab, how to culture cells and perform the assays - and for being infinitely helpful during my first years in the lab.

Tarjei S. Mikkelsen for a fruitful collaboration giving us access to new technologies - and also for hosting me in his group at the Broad and Harvard for a 
couple of months, allowing me to experience first hand the vibrant atmosphere of research in the heart of Boston.

Asbjørn Årøen, Sverre Løken and Stig Heir for teaching me rabbit surgery, for discussions on the clinical aspects of cartilage repair, and for help with bone marrow harvesting.

Finn P. Reinholt for excellent guidance with histology interpretations, good discussions and for reminding me of the importance of pilot studies.

Esben Østrup for great help in making sense of large datasets and the opportunity to practice (when I remember) my almost forgotten native tongue.

I am also thankful to Jan O. Gordeladze for helping me getting funded by the University and for the opportunity to include teaching medical students as part of the job - and for numerous discussions on science, book chapters, music and life in general.

I would like to express my gratitude to the University for funding my $\mathrm{PhD}$ and to the Norwegian Center for Stem Cell Research, the Institute of Basic Medical Sciences, and the Department of Immunology at Oslo University Hospital for providing the frame work needed for the research presented here.

Also thanks to my co-author Xiaolan Zhang at the Broad Institute for all help with the digital mRNA-profiling.

Phillippe Collas and all past and present members of his group, with whom we share the floor in the Department of Biochemistry, deserve a special thank you for collaborative projects published and unpublished, for good discussions at the coffee machine, and for all help and assistance during my time here.

Last, but not least, I have had many fine colleagues over the years: Karen Johanne Beckstrøm, Shivali Duggal, Katrine B. Frønsdal, Krisztina Szöke, Kristin Marshal, Janke B. Eriksen, Axel M. Küchler, Sarah R. Herlofsen, Torill Høiby, Amilton Fernandes, Jardar H. Stendal, Lars F. Moen, Magnus Ø. Olderøy, Rua Nader AlModawi, Jim E. Lamppu, Nanthiny Aune, Margareth V. Tamburstuen, and Xiaolin Wang. Some have left the group and some are still around, but you have all been 
helpful whenever I needed help, and even more important, have made it great to come to work every day.

Finally, thank you, Anja Maria for being my absolute best friend in love and life. I am so very glad that you found me. And to Sigrid and Bjørn: You constantly remind me what really matters - and for that I am eternally thankful.

Rune Jakobsen

Oslo, January 9th 2014 


\section{List of papers included}

Paper I:

Jakobsen RB, Shahdadfar A, Reinholt FP, Brinchmann JE. Chondrogenesis in a hyaluronic acid scaffold: comparison between chondrocytes and MSC from bone marrow and adipose tissue. Knee Surg Sports Traumatol Arthrosc.

2010;18(10):1407-16. PubMed ID:20020100.

Paper II:

Løken S, Jakobsen RB, Aroen A, Heir S, Shahdadfar A, Brinchmann JE, Engebretsen L, Reinholt FP. Bone marrow mesenchymal stem cells in a hyaluronan scaffold for treatment of an osteochondral defect in a rabbit model. Knee Surg Sports Traumatol Arthrosc. 2008;16(10):896-903. PubMed ID: 18592218.

Paper III:

Jakobsen RB, Karlsen TA, Mikkelsen TS, Brinchmann JE. microRNA-140 targets RALA and regulates chondrogenic differentiation of human mesenchymal stem cells by translational enhancement of SOX9 and ACAN. Stem Cells Dev. 2013. PubMed ID:24063364.

Paper IV:

Jakobsen RB, Østrup E, Zhang X, Mikkelsen TS, Brinchmann JE. Analysis of the effects of five factors relevant to in vitro chondrogenesis using factorial analysis and high-throughput mRNA-profiling. 2013. Manuscript submitted. 


\section{Abbreviations}

\begin{tabular}{|c|c|}
\hline ACAN & aggrecan \\
\hline $\mathrm{ACI}$ & autologous chondrocyte implantation \\
\hline ACL & anterior cruciate ligament \\
\hline ADAMTS5 & $\begin{array}{l}\text { a disintegrin-like and metallopeptidase with thrombospondin type } 1 \\
\text { motif } 5\end{array}$ \\
\hline ALPL & alkaline phosphatase liver/bone/kidney \\
\hline ANOVA & analysis of variance \\
\hline AT & adipose tissue \\
\hline BGLAP & bone gamma-carboxyglutamate protein (osteocalcin) \\
\hline $\mathrm{BM}$ & bone marrow \\
\hline BMP & bone morphogenetic protein \\
\hline CBFA & core-binding factor alpha 1 \\
\hline CCI & characterised chondrocyte implantation \\
\hline $\mathrm{CD}$ & cluster of differentiation \\
\hline cDNA & complementary DNA \\
\hline CMV & cytomegalovirus \\
\hline cMYC & v-myc avian myelocytomatosis viral oncogene homolog \\
\hline COL10A1 & collagen type $\mathrm{X}$ alpha 1 \\
\hline COL2A1 & collagen type II alpha I \\
\hline COMP & cartilage oligomeric protein \\
\hline CXCL12 & chemokine ligand 12 \\
\hline dGEMRIC & delayed gadolinium enhanced MRI \\
\hline DNA & deoxyribonucleic acid \\
\hline DNPEP & aspartyl aminopeptidase \\
\hline ECM & extracellular matrix \\
\hline EDTA & ethylenediaminetetraacetic acid \\
\hline ELISA & enzyme-linked immunosorbenta assay \\
\hline ERG & v-ets avian erythroblastosis virus E26 oncogene homolog \\
\hline ERK & extracellular signal-regulated kinase \\
\hline $\mathrm{ESC}$ & embryonic stem cell \\
\hline ETS & erythroblast transformation-specific \\
\hline FACIT & fibril-associated collagen with interrupted triple helices \\
\hline FBS & foetal bovine serum \\
\hline FDR & false discovery rate \\
\hline GAG & glycosaminoglycan \\
\hline GAPDH & glyceraldehyde 3-phosphate dehydrogenase \\
\hline GPI & glycosylphosphatidylinositol \\
\hline GTP & guanosine triphosphate \\
\hline HDAC & histone deacetylase \\
\hline HLA & major histocompatibility complex \\
\hline hPLP & human platelet lysate (enriched) plasma \\
\hline
\end{tabular}




$\begin{array}{ll}\text { ICRS } & \text { International Cartilage Repair Society } \\ \text { IFN } & \text { interferon } \\ \text { IHH } & \text { Indian hedgehog } \\ \text { iPS } & \text { induced pluripotent stem (cell) } \\ \text { ISCT } & \text { International Society for Cellular Therapy } \\ \text { ITM2A } & \text { integral membrane protein 2A } \\ \text { KLF4 } & \text { Kruppel-like factor 4 } \\ \text { LIPA } & \text { lipase A, lysosomal acid, cholesterol esterase } \\ \text { MACI } & \text { matrix-induced autologous chondrocyte implantation } \\ \text { MAPC } & \text { multipotent adult progenitor cell } \\ \text { MIAMI } & \text { marrow-isolated adult multilineage inducible (cell) } \\ \text { miR } & \text { microRNA } \\ \text { MMP } & \text { matrix metalloproteinase } \\ \text { MRI } & \text { magnetic resonance imaging } \\ \text { mRNA } & \text { messenger-RNA } \\ \text { MSC } & \text { mesenchymal stem (or stromal) cell } \\ \text { NCAM } & \text { neural cell adhesion molecule } \\ \text { NSAID } & \text { non-steroidal anti-inflammatory drug } \\ \text { OATS } & \text { osteochondral autograft transfer system } \\ \text { OCT4 } & \text { POU class 5 homeobox 1 } \\ \text { OMIM } & \text { online mendelian inheritance in man } \\ \text { PAX1 } & \text { paired box 1 } \\ \text { PCA } & \text { principal component analysis } \\ \text { PTHRP } & \text { parathyroid hormone-related protein } \\ \text { RALA } & \text { v-ral simian leukemia viral oncogene homolog A } \\ \text { RISC } & \text { RNA-induced silencing complex } \\ \text { RNA } & \text { ribonucleic acid } \\ \text { RT-qPCR } & \text { revers transcriptase quantitative polymerase chain reaction } \\ \text { RUNX } & \text { runt related transcription factor } \\ \text { SHH } & \text { sonic hedgehog } \\ \text { SiRNA } & \text { small interfering-RNA } \\ \text { SMAD } & \text { SMAD protein/gene family } \\ \text { SOX } & \text { sex determining region Y } \\ \text { SUMMIT } & \text { Superiority of MACI versus Microfracture for Treatment of } \\ \text { TGF } & \text { symptomatic articular cartilage defects } \\ \text { TGFBR1 } & \text { transforming growth factor } \\ \text { THBS2 } & \text { thrombospondin 2 } \\ \text { TMEM119 transmembrane protein 119 } \\ \text { UTR } & \text { untranslated region } \\ \text { VEGF } & \text { vascular endothelial growth factor } \\ \text { WNT } & \text { wingless-type MMTV integration site family } \\ \text { WWP2 } & \text { WW domain containing E3 ubiquitin protein ligase 2 } \\ & \end{array}$




\section{Introduction}

\section{Cartilage biology}

\section{Cartilage embryology, anatomy, and physiology}

Cartilage exists in several different forms in the human body: hyaline, elastic, and fibrous cartilage. The main focus of this thesis is hyaline cartilage and how to repair it. Hyaline cartilage is often referred to as articular cartilage, as this connective tissue is found covering the articulating surfaces in synovial joints and serves to absorb shock and allow for smooth movements. Briefly, what distinguishes the three main types of cartilage from each other are the constituents of the extracellular matrix (ECM). The hyaline ECM consists of collagens and proteoglycans but has no elastic component. Elastic cartilage is found mainly in the epiglottis, the external ear and the auditory tube, with the ECM consisting of elastic fibres in addition to the same components as in hyaline ECM. Fibrous cartilage can be considered a transitional tissue between dense connective tissue and hyaline cartilage and is mainly found in relation to joints in menisci and intervertebral discs. The ECM of fibrous cartilage differs from that of hyaline cartilage mainly in the type of collagens deposited (Ross, 2003).

Articular cartilage averages $2.4 \mathrm{~mm}$ in total height in the human knee. It consists of cells, ECM and water and has a distinct histoanatomical organisation (Figure 1) (Hunziker, 2002b). Traditionally, cartilage is divided into four different zones from the surface down to the subchondral bone. These four zones have clear structural differences. The superficial zone lines the joint cavity and is bathed in synovial fluid. In this zone the chondrocytes are flattened, with a discoid structure that aligns parallel to the surface in a matrix of thin collagen fibrils, which at least in early life consists of type I collagen (Treilleux, 1992; Bland, 1996; Tallheden, 2006). There is a relatively low proteoglycan content, but the chondrocytes 


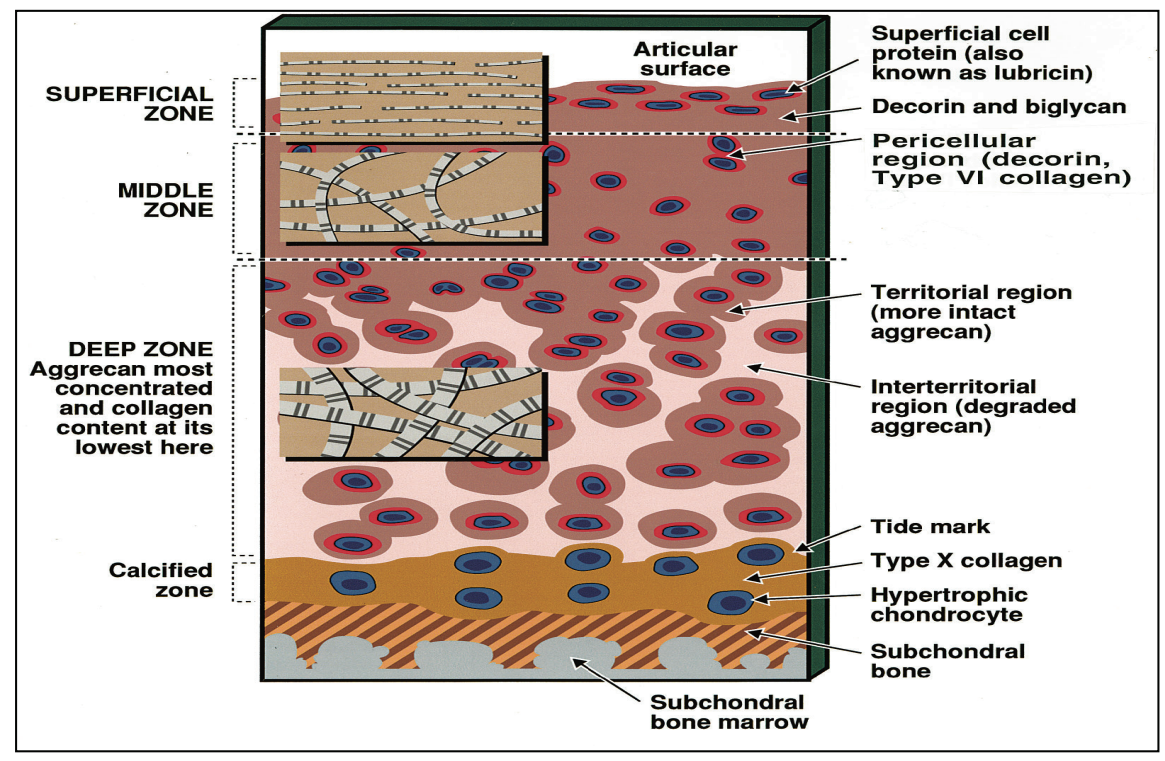

Figure 1. Schematic representation of the general structure of human articular cartilage from an adult, showing the zones, regions, and relationships with subchondral bone. The insets show the relative diameters and organisations of collagen macrofibrils in the different zones. From (Poole, 2001)

produce lubricin (or superficial zone protein) (Flannery, 1999), which is important in providing the almost frictionless articulation in diarthrodial joints (Poole, 2001). A few publications have found evidence that chondrocyte progenitor cells can be found within the superficial zone and that these cells persist throughout life (Alsalameh, 2004; Dowthwaite, 2004). Below the superficial zone is the transitional (or middle) zone, with more oval-shaped chondrocytes, lower cell density and higher proteoglycan content in a randomly distributed type II collagen network. The next zone is the deep (or radial) zone, with the lowest cell density but the highest proteoglycan content and with the collagen type II fibres oriented perpendicular to the surface. Here the chondrocytes are situated in a column-like pattern also known as palisades. At the transition between bone and cartilage we find the calcified zone just above the subchondral bone plate, marking the border to the bone marrow. Cells here usually express the hypertrophic phenotype, producing type $\mathrm{X}$ collagen and calcify their surroundings, not unlike bone 
formation but with no subsequent osteoblast and vascular invasion. The border between the uncalcified and the calcified zone is often referred to as the tidemark. The metabolism of mature articular cartilage is believed to be very low, with major collagens having a half-life of more than 100 years (Hills, 1940; Verzij1, 2000; Goldring, 2012). However, cartilage is not completely quiescent; pericellularly, there is active turnover of collagen and proteoglycan (Aurich, 2002), and during growth of articular cartilage, this happens appositionally from the surface and downwards (Hayes, 2001).

The cells of the cartilage, the chondrocytes, account for only approximately $2 \%$ of the volume of adult human articular cartilage (Poole, 2001; Hunziker, 2002b). Each individual chondrocyte is a fairly small cell, averaging $13 \mu \mathrm{m}$ in diameter. The mean cell volume is approximately $1750 \mu \mathrm{m}^{3}$ with considerable variations between zones. The cell height and cell density of articular cartilage show large variations between species, but the number of cells underlying $1 \mathrm{~mm}^{2}$ of the surface is amazingly consistent averaging approximately 25,500 cells (Stockwell, 1971). Interestingly, articular chondrocytes form functional gap junctions with neighbouring cells, but the physiological significance of this is not clear (Mayan, 2013). The term chondron has been coined for the chondrocyte and the immediate pericellular ECM. The chondron has a distinct anatomical organisation with a pericellular glycocalyx enclosed by a fibrillar type VI collagen capsule (Poole, 1997). It is speculated that chondron remodelling plays an important role in the early development of osteoarthritis (Poole, 1997).

The chondrocyte is solely responsible for producing all the constituents of the $\mathrm{ECM}$, and even in the light of the very low but existing turnover of the ECM, this makes the chondrocyte quite metabolically active (Aurich, 2002). This is evidenced by ultrastructural analysis of chondrocytes, showing a highly developed endoplasmatic reticulum and Golgi complex and large intracellular accumulation 
of glycogen and lipids (Geneser, 1999). Chondrocytes have phagocytic and autophagocytic abilities, although these features are poorly understood (Aguiar, 1999; Castillo, 2004). The chondrocyte also has a highly developed actin cytoskeleton, at least in vitro but probably also in vivo (Benjamin, 1994). The cytoskeleton may be an important mediator between cell-cell/cell-matrix interactions influencing the physiology and the differentiation of the cell acting through integrin-linked kinases and Rho GTPases (Woods, 2007).

Previously it was believed that chondrocytes in mature cartilage did not replicate at all. The discovery of progenitor cells in the superficial zone has challenged this (Dowthwaite, 2004), in line with some old reports of mitosis seen in normal (Crelin, 1957) and osteoarthritic cartilage (Rothwell, 1973). However, no one has been able to establish to what extent such turnover occurs in vivo.

The formation processes of mature articular cartilage during embryogenesis are beginning to be elucidated (Goldring, 2006b). They start shortly after gastrulation (the process establishing the three germ layers of the developing embryo) in the third week of gestation. Paraxial mesoderm along the neural tube condenses into paired segments known as somites, which ultimately develop into vertebrae. Early in the fourth week, the cells in the ventral and medial walls of the somite lose their tight organisation and give rise to a loosely woven tissue known as the sclerotome, the start of the axial skeleton. The bones of the limbs are initiated by similar cells to that of the sclerotome found in the somatic mesoderm derived from the lateral plate mesoderm (Olsen, 2000). The dorsolateral part of the somite differentiates into the dermomyotome, the source of the muscular system and the dermis and subcutaneous tissues of the skin (Sadler, 2006). The condensation process is not fully understood. It is initiated by altered mitotic activity with subsequent aggregation of cells towards a centre made possible by elimination of the abundant hyaluronic acid present in the developing somite (Hall, 1995). The further 
differentiation of the somite into the sclerotome and dermomyotome is induced by signals from the surrounding tissues. Sonic hedgehog $(\mathrm{SHH})$ is secreted by the notochord and is responsible for the induction of the transcription factor PAX1, which is highly expressed in the sclerotome and which later in the differentiation process induces SOX9, a transcription factor necessary for chondrogenesis. SOX9 is tightly involved in the regulation of type II collagen production. This enables the cells of the sclerotome to lay down a cartilage template for the whole skeleton, stabilise the embryo and serve as a template for myo- and neurogenesis. Most of this initial template is replaced by bone in a process known as endochondral ossification, wherein chondrocytes switch from a type I/II collagen production phenotype into the hypertrophic type X collagen production phenotype. Most of them subsequently undergo apoptosis. Calcification started by the hypertrophic chondrocyte is then continued by invading osteoblasts supported by angiogenesis in the primary ossification centres (Olsen, 2000).

On the actual cavitation of joints, little is known about the initiating molecular mechanisms. First an increase in cell density is seen in the area of the forming joint (the interzone), followed by dedifferentiation of the chondrocytes in the area, which switch to type I collagen production, possibly induced by the expression and secretion by cells in the area of the glycoprotein WNT9A (previously known as WNT14) (Bland, 1996; Hartmann, 2001). Cells in the interzone area become elongated and endothelial-like, and in the middle of the zone, cells are progressively lost through apoptosis, thereby forming the joint cavity. It is believed that movement of the joints occurs through extracellular signal-regulated kinases (ERKs), a subgroup of the mitogen-activated protein kinase pathway, to modulate the production and secretion of ECM components, especially hyaluronic acid. Hyaluronic acid subsequently facilitates a condensation process similar to the one outlined for the somites, leading to differentiation of these cells into mature chondrocytes and to the formation of the articular cartilage. 
Articular cartilage is non-vascularised and non-innervated. The calcified zone is practically impermeable to gases and fluids, so nutrition of the cells in cartilage occurs by diffusion of substances from the synovial fluid to the cells. Synovial fluid itself is low in oxygen compared to arterial blood, leaving articular cartilage severely hypoxic compared to vascularised tissues. The anatomical properties likely do not allow any cell migration within the cartilage. These physiological and anatomical properties possibly form the basis of the empiric fact that articular cartilage, once injured, does not heal (Hunter, 1742), unless the injury penetrates the subchondral bone-plate, after which partial repair often happens, though not with histologically normal cartilage (Hunziker, 2002a).

\section{In vivo chondrogenesis and endochondral ossification}

Embryological chondrogenesis has been depicted above, but it is worthwhile to delve a little deeper into the factors that have been established as important in each step of chondrogenesis from immature stem cell to mature and then to hypertrophic chondrocyte. Much of this knowledge is derived from studies of chicken and mouse and more recently from human in vitro cultures of differentiating embryonic and adult stem cells (Goldring, 2006b; Nakagawa, 2009; Yamashita, 2010). Chondrogenesis is a complex process tightly modulated by several identified factors. Figure 2 gives an overview of what is known to regulate each step in chondrogenesis. The prechondral mesenchymal cell produces an ECM rich in hyaluronic acid and type I collagen, which allows mesenchymal cells to migrate to the site of future skeletogenesis dependent on hyaluronidase activity. This initiates the condensation process, with the appearance of cell adhesion molecules 


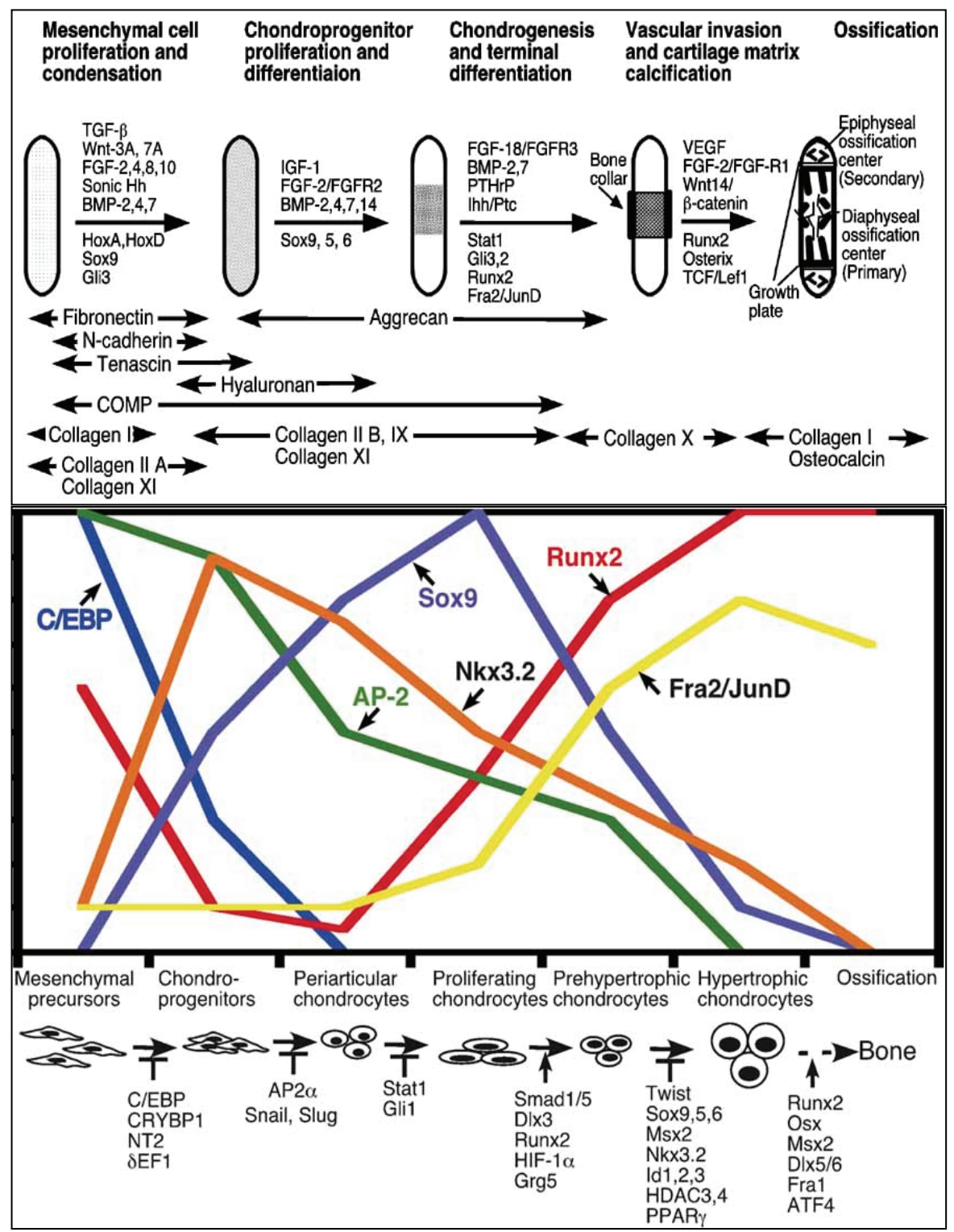

Figure 2. Top panel: The different stages of chondrogenesis represented schematically, showing the temporal patterns of growth and differentiation factors (above the arrows) and the transcription factors involved (below the arrows). The ECM proteins that distinguish the different stages are indicated below. Bottom panel: Schematic representation of the expression of transcriptional regulators at different stages of chondrogenesis and endochondral ossification. Additional transcription factors that are inhibitors or activators at different stages are indicated below the scheme of cellular transitions (from (Goldring, 2006b)). 
such as neural cadherin and neural cell adhesion molecule (NCAM). NCAM expression is regulated by fibronectin production, which in turn is closely related to the cell's exposure to transforming growth factor beta (TGF $\beta$ ) (discussed later). The condensation process marks the start of chondrocyte differentiation, which is characterised mainly by the differences in the type of collagen deposition. From Figure 2 it can be seen that several transcription factors are up- and downregulated in the differentiation process (Goldring, 2006b). One of the earliest and arguably the most important transcription factor is $\mathrm{SOX} 9$, which is required for the expression of type II collagen (Bi, 1999). SOX9 is the master regulator of chondrogenesis (Foster, 1994). The importance of SOX9 for chondrogenesis is most clearly illustrated by the autosomal-dominant disease campomelic dysplasia (OMIM \#114290) caused by a heterozygous SOX9 mutation, which often leads to perinatal death due to poorly developed cartilage in the airways (severe laryngotracheomalacia). Other distinct features of individuals with campomelic dysplasia include craniofacial defects, deformed pelvis, long bones, and ambiguous genitalia (Yap, 2011). Correspondingly, Sox9-haploinsufficient $\left(\operatorname{Sox} 9^{+/}\right)$mice die perinatally, and complete knockout $\left(\operatorname{Sox} 9^{-/}\right)$mice die in early embryonic life (Bi, 1999; Akiyama, 2002). Conditional knockout experiments where one or both alleles can be turned off either globally or in chondrogenic tissue (i.e., Col2a1-expressing tissue) at specific timepoints during development have shown that Sox9 is needed for mesenchymal condensation and that loss of Sox9 leads to enlargement of the hypertrophic zone and premature mineralisation (Kist, 2002; Yap, 2011; Dy, 2012).

SOX9 works in chondrogenesis as a homodimer (Bernard, 2003) that can be further stabilised by two other SOX proteins from the SoxD family: L-SOX5 and SOX6 (Yamashita, 2012). These factors are co-expressed with SOX9 during differentiation and are linked to production of type II and IX collagen as well as the major proteoglycan aggrecan (Lefebvre, 2001). Their importance is illustrated 
by other knockout experiments showing that while Sox5- or Sox6-single-null mice are born with relatively mild abnormalities but do die in early life, double-null mice die in utero with severe chondrodysplasia (Smits, 2001; Akiyama, 2008). Recently, a fourth SOX protein, SOX8, has been identified as a possible regulator of chondrogenesis (Herlofsen, 2011; Herlofsen, 2014). Another transcription factor of major importance is the runt-domain transcription factor RUNX2, also known as core binding factor alpha 1 (CBFA1). This factor is upregulated later in chondrogenesis and leads to hypertrophy, wherein the cells increase in size and shift to type $\mathrm{X}$ collagen production. These cells are seen in the deep calcified zone of articular cartilage and, importantly when considering tissue engineering, in the part of the early cartilaginous skeleton destined to be invaded by osteoblasts. Not surprisingly, RUNX2 is also important for osteoblast and osteoclast maturation by regulating the proangiogenic VEGF, the matrix degrading/remodellating MMP13 and MMP9 and the mineralisation protein ALPL (Stickens, 2004; Colnot, 2005; Yoshida, 2005). Also SOX8 has been shown to inhibit MMP13 and ALPL perhaps playing a crucial role in preventing hypertrophy (Herlofsen, 2014). It is speculated that the relationship between SOX9 and RUNX2 ultimately determines which parts of cartilage stay cartilage and which parts become bone. One example of this is that parathyroid hormone-related peptide (PTHRP), present in the growth plate of long bones, prevents chondrocytes from going into the hypertrophic state, likely because PTHRP phosphorylates SOX9, increasing its affinity to DNA (de Crombrugghe, 2001; Huang, 2001). There is also evidence that SOX9 itself is necessary for COL10A1 expression, though it also prevents osteoblastic differentiation of prehypertrophic chondrocytes (Dy, 2012).

Several other factors are important in chondrogenesis, among them the secreted peptide Indian hedgehog (IHH) from prehypertrophic chondrocytes exposed to low levels of PTHRP. IHH in turn induces PTHRP expression, resulting in cells being kept prehypertrophic (St-Jacques, 1999). Two splice variants of the ETS- 
related gene $(\mathrm{ERG})$ play a role in determining the functional role of developing cartilage (Iwamoto, 2000; Iwamoto, 2007).

\section{In vitro de- and redifferentiation of chondrocytes}

The term dedifferentiation of chondrocytes is used for the shift in ECM production and morphology seen when articular chondrocytes are expanded in a monolayer (Holtzer, 1960). When chondrocytes after enzymatic digestion of the ECM are put into culture flasks, they rapidly adhere to the plastic and change into a fibroblastlike shape, with long and eventually entangled cell extensions. At the same time, a change from mainly type II collagen to type I collagen production and from primarily aggrecan to versican production occurs. The consensus has been that this represents chondrocyte dedifferentiation into a more primitive state characteristic of the early stages of embryogenic chondrogenesis. This has to some extent been substantiated by findings that a change in cell surface markers occurs that makes cultured chondrocytes fairly similar to the immunophenotype of cultured mesenchymal stem cells (MSCs) (Diaz-Romero, 2005). And some studies have shown that cultured chondrocytes do have a limited but existing ability to differentiate into other mesenchymal lineages, such as osteocytes and adipocytes (Tallheden, 2006). Some evidence has emerged showing that the phenotype of chondrocytes is actually modulated over time in culture and is not the result of a selective expansion of progenitor/stem cells (Diaz-Romero, 2007). It has also been shown that culturing chondrocytes after only a short enzyme treatment that leaves some of the ECM intact, delays the dedifferentiation process (Shahdadfar, 2008). Nevertheless, it is poorly understood what drives the change of phenotype and ECM production and exactly which signals are responsible for the change. So far, there are no completely successful systems for redifferentiation of chondrocytes into hyaline cartilage. Chondrogenic protocols devised for stem cell differentiation can be used with success on chondrocytes (Fernandes, 2013), though with some of the same problems as for stem cells (discussed later). 


\section{Extracellular matrix}

Basically, the ECM can be viewed as the structural framework outside the cells, with all its associated molecules. The structural framework makes up approximately $90 \%$ of the dry weight of articular cartilage and can be subdivided into collagens, proteoglycans and non-collagenous proteins and glycoproteins (Seibel, 2006).

\section{Collagens}

Two-thirds of the dry weight of articular cartilage is collagen of various types. At least 27 types of collagen are expressed in higher vertebrates, the products of more than 40 genes (Eyre, 2004). The key feature of all collagen types is the triple-helix structure. It is a coiled-coil structure composed of three polypeptide chains ( $\alpha$ chains) with glycine in every third position (Seibel, 2006). All fibril-forming collagens are synthesised as procollagen chains that assemble in the endoplasmatic reticulum to a 300-nm-long triple helix. The procollagen is then secreted and the propeptides removed by specific enzymes, which initiates actual fibril formation from cross-linking of several collagen molecules. Fibrils can then aggregate into actual collagen fibres. High amounts of the amino acids proline and hydroxyproline allow for extensive cross-linking within and between each collagen molecule (Alberts, 2002). The amount of cross-linking is the basis for the tensile strength of collagen and varies highly between tissues and is especially high in the Achilles tendon. Type II collagen is the main type of collagen found in articular cartilage, accounting for about $90 \%$ of the total collagen. Each collagen molecule consists of three identical $\alpha 1[\mathrm{II}]$-chains that assemble into fibrils. Type II collagen is most abundant in the radial zone. It exists in four splice variants (McAlinden, 2008). Type IIB is found in mature cartilage, where exon 2, coding for 69 amino acids, is spliced out, and type IIA is found together with type IID in embryonic cartilage, where exon 2 is retained. Type II collagen forms a copolymer with the smaller type XI collagen and with type IX collagen, the latter a so-called fibril-associated collagen with interrupted triple helices (FACIT) which facilitates 
fibril formation (Eyre, 2004). Another important type of collagen normally only found in very small amounts in the superficial region of mature cartilage, is type I collagen. It is probably the most studied collagen and consists of two $\alpha 1$ (I) and one $\alpha 2(\mathrm{I})$-chains. It is the most important collagen in skin, bone, tendon and fibrous cartilage (e.g., menisci and intervertebral discs). It is also present in high concentrations during embryogenesis and plays a role as an adhesive substrate for several cells in tissue and organ development; in cell migration, proliferation and differentiation; and in wound healing (Seibel, 2006). More important in terms of articular cartilage, it is the major collagen found in the tissue seen after spontaneous repair of osteochondral injuries. Type $\mathrm{X}$ collagen is a so-called network-forming collagen. It is a short-chain collagen that forms a pericellular network associated with macrofibrils of type II collagen. The function of type X collagen is not fully understood, but it plays a role in endochondral ossification. It may be involved in avoiding actual bone formation in calcified cartilage yet at the same time involved in matrix-vesicle-initiated calcification of hypertrophic cartilage (Kwan, 1997; Poole, 2001). Phenotypic changes in two type X collagen knockout strains of mice were surprisingly small compared to the severe joint defects seen in the human disease Schmid-type metaphyseal chondrodysplasia (OMIM \#156500), which is caused by a mutation in the type X collagen gene (Seibel, 2006). The actual function of another collagen, type VI, is also unknown, but it forms the fibrillar pericellular network in the chondrons (Poole, 1997). In knockout mice, type VI collagen loss is linked to altered trabecular bone structure and delayed cartilage degeneration (Christensen, 2012).

In summary, the fibril-forming collagens, regardless of the tissue, are responsible for the tensile strength of the tissue. They also provide a framework that contains the pressure from swelling due to embedded proteoglycans. All collagens may exert biological effects on the cells through integrins, although this is far from understood. 


\section{Proteoglycans}

The other major component of the structural framework of articular cartilage is the proteoglycans. They form a special class of glycoproteins with long, unbranched, and highly charged glycosaminoglycan (GAG) chains attached. Due to their charge, the GAG chains are highly hydrophilic, and as they are trapped within the collagen network and too large to redistribute, water is drawn into the cartilage by osmosis. This swells the ECM until equilibrium between the tensile strength of the

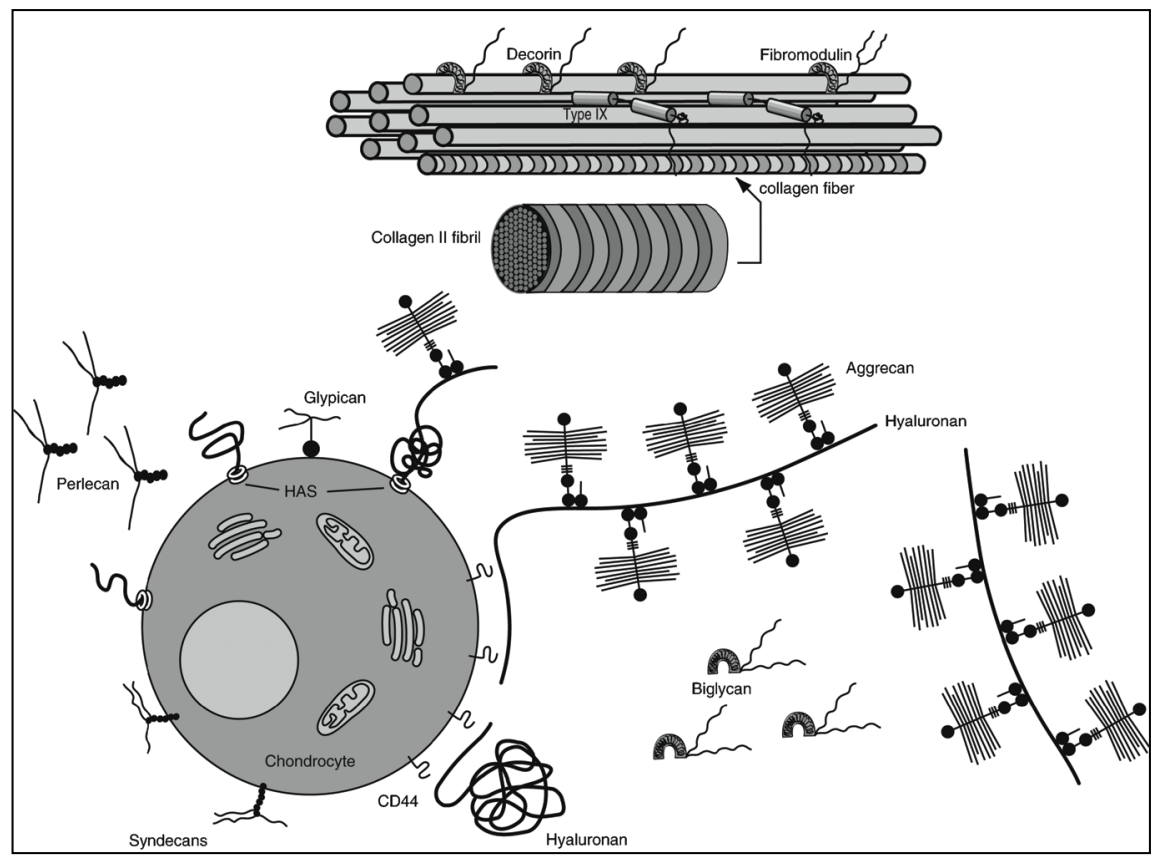

Figure 3. Overview of the proteoglycans present in cartilage. Associated with the cell surface are the transmembrane-spanning syndecan proteoglycans, the GPI-linked heparan sulphate proteoglycan, glypican, and two forms of hyaluronan, namely hyaluronan bound to the hyaluronan synthase and hyaluronan tethered to CD44. Aggrecan binds to cell surface-associated hyaluronan as well as hyaluronan within the further removed ECM. Several small proteoglycans, namely decorin, fibromodulin and type IX collagen, form strong associations with cartilage collagen fibrils (types II, IX and XI collagens). Other proteoglycans, such as biglycan and perlecan, are also present within cartilage, but their localisation and binding partners have not been firmly identified. From (Knudson, 2001). 
collagen network and the water is reached. Any external force applied to the cartilage will then additionally increase the pressure within the cartilage, but since fluids are incompressible this gives the cartilage an excellent compressive stiffness. The strong charges of the GAG chains also highly diminish fluid flow within the cartilage, making the whole cartilage fairly resistant to impact loading, although constant sustained loads will eventually force a redistribution of fluid (Seibel, 2006).

Aggrecan is the main proteoglycan of cartilage and has a core protein of high molecular weight ( $250 \mathrm{kDa})$, with three globular and two extended domains. The extended domains are highly glycosylated by two types of GAG chains, chondroitin sulphate ( $\sim 100$ per aggrecan molecule) and keratan sulphate ( $\sim 60$ per molecule). Hyaluronan (also termed hyaluronate or hyaluronic acid) is a long, unbranched glycosaminoglycan based on a disaccharide repeat of glucosamine and glucuronate found in especially high concentration in embryonic cartilage. It has no protein directly associated and is therefore not a proteoglycan per se, but in mature cartilage it forms aggregates with aggrecan molecules, as these contain highly specific binding sites with high affinity for hyaluronan. This binding is stabilised by link protein, a member of a small gene family of proteins tightly linked to aggrecan expression, yet the function of the members of this family is largely unknown. Hyaluronan has an undefined interaction with the collagen fibrils and a defined attachment to the cell surface receptor CD44 present on chondrocytes. The function of this binding is not well understood. It is known that there is a normal turnover and internalisation of CD44 bound to hyaluronan with subsequent degradation of hyaluronan. The internalisation of CD44 is upregulated if cells are stripped of attached hyaluronan (Aguiar, 1999; Knudson, 2001). It is also known that if blocking of the CD44 receptor occurs by small hyaluronan fragments, a chondrolytic state is induced (Knudson, 2002). This does not happen if the hyaluronan is larger (higher molecular weight, i.e., longer chain of 
polysaccharides), as in the commonly occurring hyaluronan in articular cartilage. Unlike the long lived collagen fibrils, aggrecan is synthesised and secreted continuously (Knudson, 2001).

Other smaller proteoglycans important to articular cartilage are the leucine-rich proteoglycans decorin, fibromodulin, lumican, biglycan and perlecan. These are all non-aggregating proteoglycans. Decorin, fibromodulin and lumican play roles in the formation and stability of collagen macrofibrils and may have some regulatory functions on the chondrocytes, although this is not fully elucidated. Both decorin and biglycan bind TGF $\beta$, which may be part of their regulatory functions (Poole, 2001). Perlecan is a somewhat larger, multidomain proteoglycan that may self-aggregate and localises to the cell surface, possibly through interaction with integrin. Addition of perlecan to chondrocyte cell cultures leads to re-expression of the type II collagen-producing phenotype, maybe working in concert with the transcription factor SOX9 (Knudson, 2001; Seibel, 2006).

\section{Other components}

Several other molecules not in the above groups are present in cartilage. Some have already been mentioned, such as lubricin and link protein. Others include the five-armed cartilage oligomeric matrix protein (COMP) of the thrombospondin family, matrilins, fibronectin, fibrilins, and chondroadherins. Little is known about the functions of these non-collagenous non-proteoglycans. COMP binds type II collagen and may be involved in macrofibril assembly. It has been used as a marker in synovial fluid of cartilage degradation in the progression of osteoarthritis, although its specificity may not be good, as COMP is also present in the synovium. Fibronectin is an important protein in early chondrogenesis and embryogenesis and is present in mature cartilage at low levels. Interestingly fibronectin is upregulated in early and late osteoarthritis (Lorenzo, 2004). Chondroadherins may serve to anchor chondrocytes to collagen fibrils (Seibel, 2006). 


\section{Cartilage injuries and treatment}

Cartilage is vulnerable to degenerative or traumatic damage. Diseased cartilage represents a major cause of chronic disability in developed countries (Centers for

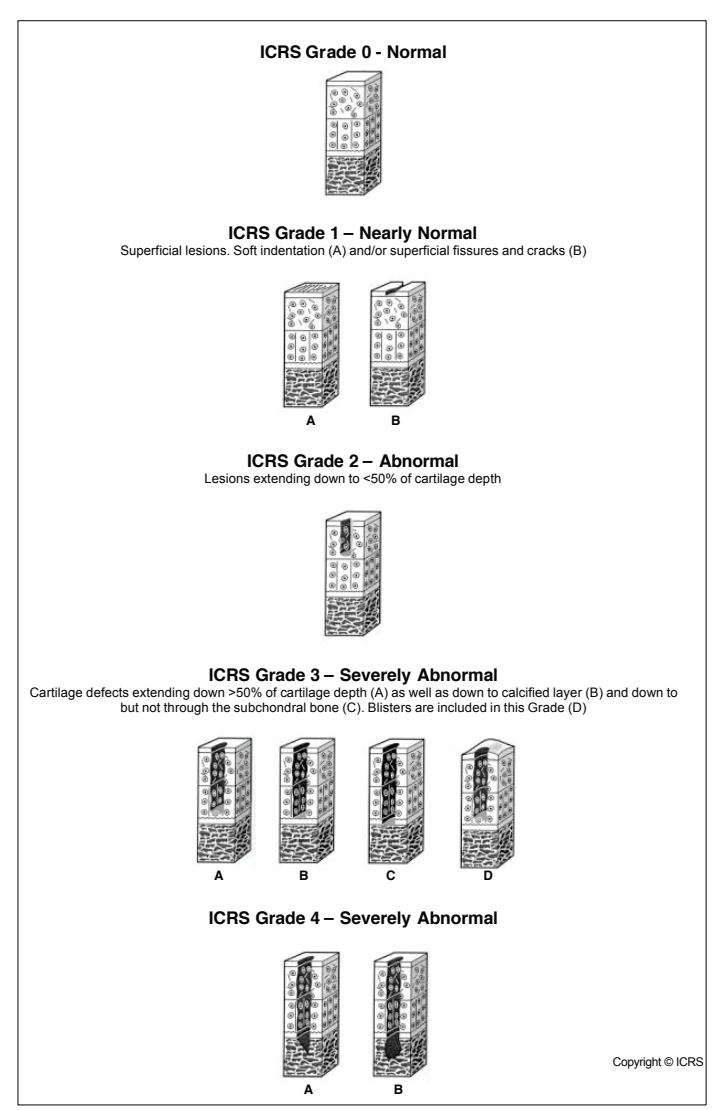

Figure 4. The International Cartilage Repair Society Injury Classification Scheme. See text for explanation. From (ICRS, 2000).
Disease Control And Prevention, 2010). Once injured, cartilage has a limited healing capacity due to its non-innervated and non-vascularised nature. Traditionally, we divide cartilage pathology into traumatic (or focal) injury and degenerative changes (the latter either primary or secondary). Cartilage repair is generally focused on repairing focal injuries to relieve symptoms, and hopefully also preventing the development of secondary osteoarthritis, though the latter has not been shown convincingly in clinical studies.

\section{Grading and scoring systems for cartilage injury}

By definition, a cartilage (or chondral) lesion does not penetrate the subchondral boneplate. If it does penetrate the subchondral boneplate, it is an osteochondral lesion. Several grading systems have been developed, but the most common way 
to grade chondral and osteochondral injury today is the International Cartilage Repair Society (ICRS) evaluation package first proposed in 1998, developed in several workshops in 2000 and freely available from the society, with both a patient and a surgeon part (Brittberg, 1998; ICRS, 2000; Brittberg, 2003). In this system, the macroscopic grading of the injury is based on the simple and straightforward classification scale developed by Outerbridge in 1961 (Outerbridge, 1961). The gradations and their corresponding types of injury are depicted in Figure 4. Naturally, in addition to the grade, the size of the lesion is also part of the classification. The common consensus (although without solid scientific evidence) is that a lesion larger than $2 \mathrm{~cm}^{2}$ is considered a large lesion (Behery, 2013). The ICRS evaluation system also includes a grading system for osteochondritis dissecans lesions as well as a grading system for macroscopic evaluation after cartilage repair.

Cartilage injuries are generally not easily seen on standard weight-bearing radiographs unless they are large or are part of a more generalised osteoarthritis where diminished joint space can be seen. MRI with special sequences and/or with gadolinium contrast (delayed gadolinium-enhanced MRI of cartilage, dGEMRIC) specifically quantifies GAG content of diseased portions of the cartilage (Nieminen, 2012; Palmer, 2013). This provides a promising tool for non-invasive evaluation of cartilage injury.

\section{Epidemiology}

Injuries to articular cartilage in the knee are fairly common. In a retrospective review of 31,516 knee arthroscopies, cartilage lesions were documented in approximately 20,000 patients (63\%) (Curl, 1997). In two-thirds of these there was an associated ligamentous and/or meniscal pathology. Approximately $60 \%$ of the lesions were grade 3 or worse, and the most common place to have a grade 4 lesion was the medial femoral condyle. A more recent retrospective review of 25,124 arthroscopies largely corroborated these findings (Widuchowski, 2007). 
Two other prospective studies of approximately 1,000 consecutive arthroscopies of the knee found lesions of the articular cartilage in $61 \%$ and $66 \%$ of patients, respectively, in line with a retrospective survey (Hjelle, 2002; Aroen, 2004). Localised or focal chondral and osteochondral lesions were found in both studies in approximately $20 \%$ of the knees. Full-thickness lesions (ICRS grades 3 and 4) were found in $11 \%$ of the knees, and of these, $55 \%$ were more than $2 \mathrm{~cm}^{2}$. In both studies, cartilage lesions were more frequently seen in conjunction with injuries to the cruciate ligaments and/or menisci. In the national Scandinavian registries of anterior cruciate ligament (ACL) reconstructions, cartilage injury was noted in 17$27 \%$ of all cases (Granan, 2009). This is concordant with another study that found chondral lesions in 19\% of ACL ruptures and an increased incidence of associated chondral injury with longer duration between rupture and repair (Tandogan, 2004). This last finding indicates that not all chondral injuries are apparent at the time of ligament injury, but that either the altered biomechanical properties of the knee predispose the patient to subsequent chondral injury or that a lesion may develop over time, possibly due to damage to the underlying bone sustained at the time of injury. Also, severe cartilage injury is more prevalent in athletes (Flanigan, 2010). Puzzlingly, this severe injury does not always lead to symptoms (Shellock, 2003; Walczak, 2008). These asymptomatic lesions make diagnosis and management of cartilage injury a challenging task.

\section{Pathogenesis}

Several conditions in the knee lead to chondral or osteochondral lesions and therefore there is no uniform pathogenetic pattern. In focal lesions, trauma in some form is believed to be involved. Animal and ex vivo studies of human cartilage have shown that after blunt trauma that does not initially make any macroscopic changes to the cartilage, increased apoptotic cell death is seen, along with some changes in metabolism, leading to increasing degeneration of collagen and loss of proteoglycans (D'Lima, 2001a, b). It is speculated that the bone bruise often seen on MRI after a ligament injury could indicate this type of early cartilage 
degeneration (Nakamae, 2006). It is, however, also speculated that the damage to the bone actually over time affects the overlying cartilage due to the changed biomechanical properties of the bone after injury and intrinsic repair. In more severe trauma to the knee, actual chondral fracture is seen immediately after the injury, and in even more severe trauma osteochondral fractures are seen from direct impact to the cartilage, but probably more often the traumatic episode inflicted a fracture to the bone that extends into the articular surface. There are also a few supposedly non-traumatic origins of cartilage injuries. One is the entity known as osteochondritis dissecans, where an osteochondral fragment loosens in juveniles. The patient presents with a painful knee, often with full dislocation of the fragment and a locked knee. The aetiology of this is not known, but animal studies suggest that disturbances of vascularisation of the bone in the embryonic development may predispose to this condition (Ytrehus, 2004a; Ytrehus, 2004b; Ytrehus, 2004c). Another spontaneous condition is osteonecrosis of the subchondral boneplate. This is rather uncommon but occurs in older age groups and leads to loss of the supportive function of the subchondral bone from necrosis and subsequently severely affects the overlying cartilage. It has been suggested that the underlying aetiology of this is stress fractures in the subchondral bone (Patel, 1998; Mont, 2000).

More recently, advances in molecular biology have led to discoveries of relationships between genes, cytokines and growth factors that may help to explain the pathogenesis of osteoarthritis which could also possibly play a role in the likelihood of a minor traumatic focal injury developing into more severe cartilage disease (Lee, 2013).

\section{Natural history}

The non-innervated, non-vascular nature of articular cartilage may cause chondral injuries to be initially asymptomatic. The same properties are responsible for the limited self-repair seen in chondral injuries, as no reparative inflammatory 
response can be initiated. The intrinsic properties of cartilage, in addition, allow for limited migration and proliferation of the chondrocytes (Hunziker, 2002a). Only in osteochondral injuries is self-repair with fibrous cartilage seen, which is attributed to the forming of a blood clot due to bleeding from the bone marrow and, supposedly, differentiation of MSCs from the bone marrow into type I collagen producing chondrocytes (Hunziker, 2002a).

Information on the natural history of cartilage injury is sparse. It is not known whether all cartilage injuries, chondral or osteochondral, will eventually lead to secondary osteoarthritis or if there is a critical size or depth that will predict progression. One intermediate-term follow-up on a large group of patients with arthroscopically verified non-treated chondral injury has been published (Shelbourne, 2003). This study analysed patients that had undergone ACL surgery with an incidental finding of a chondral injury (grades 3 and $4, n=125$ ), which was left untreated, and compared this with a control group also undergoing ACL surgery but with no chondral injury present. A slightly but significantly better subjective score was found in the control group, but no significant difference was present in radiographic scores. Mean follow-up was 6.3 years, and mean defect size was $1.7 \mathrm{~cm}^{2}$ (range $0.5-6.5$ ). Strictly speaking, this study is not a true natural history study, as the repair of the ACL is an intervention that possibly prevents further chondral damage (as evidenced by the higher incidence of chondral injuries in old ACL ruptures) and possibly introduces cells from the bone marrow into the joint through the tunnels drilled for the ligament graft to be fixated. It is, however, clear that in a span of more than 6 years, there was no subjective or radiographic evidence of rapidly developing secondary osteoarthritis. One should also note that the activity level of the patients included in the study was fairly low. Another study followed 28 athletes for more than 14 years (Messner, 1996). In this study the athletes had isolated traumatic chondral lesions or osteochondritis dissecans. Only limited intervention in the form of removing loose bodies and, in 
three cases, Pridie drilling was performed. 22 patients had excellent or good knee function and were involved in individual sports on a fitness level, yet 12 patients had radiographic findings of osteoarthritis in the compartment of the initial injury. This is to some extent supported by the Norwegian ACL registry, which indicated no difference in preoperative function scores in patients with or without injury (Hjermundrud, 2010).

Another study with a mean follow-up of 15.3 years of severe isolated and untreated cartilage injuries reported that these injuries had hardly any influence on clinical outcomes nor on the development of osteoarthritis, as evidenced by radiographic comparisons to the uninjured contralateral knee (Widuchowski, 2011).

\section{Treatment modalities}

The ultimate goal of a cartilage repair procedure is to reconstitute the defect with a repair tissue with similar properties to that of the original cartilage, including perfect integration into the surrounding tissue. Additionally, there should be no changes to the subchondral bone or to the surrounding tissue as a consequence of the treatment (Hunziker, 2002a). To date, a treatment that fulfils these requirements does not exist (Mastbergen, 2013). Below, I shall briefly go through several of the techniques used. The list is not exhaustive but includes the most commonly used techniques as well as some experimental treatments emerging as candidates for future therapy.

\section{Non-surgical treatment}

Every surgical procedure carries a risk. Therefore, non-surgical treatment of all injuries should always be considered and surgical procedures only performed if they positively benefit the patient in terms of prognosis with an acceptable risk of both minor and major side effects. Non-surgical treatment of symptoms due to cartilage injury includes standard analgesic treatments such as non-steroidal anti- 
inflammatory drugs (NSAIDs) and less evidence-based oral supplements of glucosamine sulphate or chondroitin sulphate (Vista, 2011). It is not known if the commonly used intra-articular injections of hyaluronic acid in the treatment of osteoarthritis is beneficial in the treatment of focal defects (Axe, 2013), though there is some evidence from animal studies that it could help heal both osteochondral and chondral defects (Miyakoshi, 2005; Jansen, 2008). Arguably the most important conservative strategy is physical therapy, aimed at improving muscle strength and neuromuscular control (Wilk, 2006). The effect of a wellplanned intensive physical rehabilitation program was perhaps most convincingly demonstrated in the Oslo Cartilage Active Rehabilitation and Education Study. Patients (all with Lysholm scores below 75) referred for cartilage repair surgery achieved very good improvement of functional scores. In fact, a significant number of patients opted to not proceed with surgery after three months of rehabilitation (Wondrasch, 2013). The long-term sustainability of this rehabilitation remains to be established.

\section{Surgical treatment}

As evident from the previous section, surgical treatment of cartilage defects should be limited to patients with symptoms consistent with a full-thickness cartilage defect where a conservative treatment has failed to achieve a result that the patient considers satisfactory.

\section{Removal of loose bodies/debridement}

The simplest surgical treatment of cartilage injury is removal of the loose part of the cartilage and careful debridement of the lesion without extending the defect deeper or penetrating the subchondral bone plate. There is little evidence on the long-term results of this treatment, although it is probably performed relatively often, especially in patients presenting with a locked joint. In patients where the loose body is due to an osteochondritis dissecans lesion, the common consensus is that attempts should be made to fixate the fragment if possible or proceed with 
cartilage repair procedures (Schenck, 1996). It is worth noting that a randomised controlled study, though later heavily debated on grounds of its inclusion criteria and statistics, found that the placebo effect of a sham arthroscopy operation equalled the effect of actual lavage or lavage and debridement in patients with osteoarthritis (Moseley, 2002).

\section{Bone marrow-stimulating procedures}

Microfracture treatment is the most frequently used bone marrow stimulating technique. Other techniques in this group are abrasion arthroplasty and subchondral drilling. The microfracture technique was introduced by Steadman and coworkers and was designed to be less invasive and presumably cause less heat necrosis to the subchondral bone plate than subchondral drilling. However, recently the latter has been challenged in laboratory studies, and new instruments for subchondral drilling has been introduced (Arthrex Powerpick ${ }^{\circledR}$ ) (Steadman, 1998; Chen, 2009; Chen, 2011a). Traditionally, microfracture is performed using a specially designed awl to manually penetrate the subchondral bone in several places 3-4 $\mathrm{mm}$ apart, leading to bleeding and formation of a fibrin clot in the defect. Clinical results have shown good improvement and good repair on secondlook arthroscopy, but biopsies show fibrocartilage (Blevins, 1998). Animal studies have also shown good filling of defects but also a possibly permanent thickening of the subchondral bone plate, the importance of which is not fully known (Aroen, 2006). Randomised clinical trials on microfractures have shown microfracture to be neither superior nor inferior to autologous chondrocyte implantation (ACI) when considering clinical outcomes (Knutsen, 2004; Knutsen, 2007; Saris, 2008; Vanlauwe, 2011).

\section{Osteochondral auto-/allograft}

Osteochondral allografts (either fresh or cryopreserved) have been used for decades to treat large defects in the knee, at first primarily after tumour surgery (Mankin et al., 1976). A 20-year follow-up showed graft survival in 66\%, and 
found that rejection reactions were not a significant problem (Shasha et al., 2002). This procedure relies on the availability of suitable donors in terms of size and absence of comorbidity. Also, there have been some cases of transfer of potentially fatal pathogenic bacteria (Centers for Disease Control and Prevention, 2002). In Scandinavia, osteochondral allografts are rarely used. Instead, autograft procedures, termed mosaicplasty or osteochondral autograft transfer system (OATS), have been used in both open and arthroscopic surgeries. The technique utilises a hollow burr to remove circular plugs of cartilage, including the subchondral bone, from uninjured parts of the knee and press fitting them into the prepared cartilage defect (Matsusue, 1993; Hangody, 1997; McCoy, 2012). In randomised controlled trials, this technique performed worse than autologous chondrocyte implantation in one study (Bentley, 2003) and better than microfracture in another study (Gudas, 2005). A third, underpowered randomised trial also showed excellent results in the mosaicplasty group (Dozin, 2005). In large case series with other authors, this technique has shown excellent results (Hangody, 2003; Hangody, 2010). The technique is generally not suitable for very large lesions and is advocated in algorithms as most suitable for lesions of approximately $1 \mathrm{~cm}^{2}$ (Vanlauwe, 2007). The concern with this technique is, naturally, that to repair one defect, one or several new defects are created in the joint, presumably in less weight-bearing areas, leading to some donor site morbidity (Matricali, 2010). It also carries challenges in achieving a normal contour of the repair.

\section{Autologous chondrocyte implantation}

In 1994 a new concept of cartilage repair emerged (Brittberg, 1994). It had been known for decades that enzymatically treated cartilage biopsies made it possible to culture and numerically expand chondrocytes in vitro (Green, 1971). Animal studies of periosteal transplantation were published, followed by human studies (O'Driscoll, 1984; Lorentzon, 1998; Alfredson, 1999). A Swedish group combined these two techniques in a procedure termed autologous chondrocyte implantation 
(ACI). In this procedure, a small cartilage biopsy is arthroscopically harvested from the knee, enzymatically treated and cultured for approximately three weeks, after which at least one million cells per square centimetre are implanted in a small-volume suspension under a periosteal flap in a second open-surgery procedure. Later, the periosteal flap has been replaced by a membrane made from bovine collagen, making the procedure simpler and alleviating the problems initially seen with hypertrophy of the repair tissue, often leading to a rearthroscopy rate of 20-25\% (Knutsen, 2004; Steinwachs, 2007). The initial study reported excellent healing in 14 out 16 patients at two years' follow-up. Since then, the ACI procedure has evolved. Second-generation procedures use a membrane as the cell carrier when the cells are implanted (such as MACI ${ }^{\circledR}$ (Verigen, Germany) (Enea, 2012). Third-generation procedures culture the cells in three-dimensional matrices before implantation. One commercially available example of this made from hyaluronic acid is Hyalograft $C^{\circledR}$ (Fidia, Italy) (Marcacci, 2005). Some of these advances have also made it possible to perform the second procedure arthroscopically.

A fairly recent Cochrane review concluded that there is no evidence that ACI of any generation performs any better than other cartilage repair techniques, based on six heterogeneous randomised controlled trials (Vasiliadis, 2010). Earlier systematic reviews also found severe limitations in the research methods used in both randomised controlled trials and case series (Jakobsen, 2005; Jakobsen, 2007). Of the trials in the Cochrane review, one trial out of three that examined ACI versus mosaicplasty statistically favoured ACI, while another found significant improvement for the mosaicplasty group using one functional scoring system but not with other scoring systems. Two trials compared ACI or a more sophisticated version, termed characterised chondrocyte implantation (CCI), with microfracture and found no significant difference on functional outcomes. But in the latter trial the results at 18 months showed a significant improvement in the structural repair 
in the CCI group and at 36 months a significant functional improvement. However, the five-year results of this trial, published after the Cochrane review, found no significant functional difference between the treatments. A subgroup analysis did show a significant improvement in patients treated with symptom onset less than 3 years (Saris, 2008; Saris, 2009; Vanlauwe, 2011). The other trial comparing ACI and microfracture did not find significant differences at two or five years posttreatment (Knutsen, 2004; Knutsen, 2007). One recently published abstract of the SUMMIT randomised controlled trial comparing MACI with microfracture states that MACI achieves significantly better functional results at two years, especially when lesions were larger than $4 \mathrm{~cm}^{2}$, though details are sparse (Saris, 2013). Also with ACI, there are concerns about donor site morbidity in the site of the initial cartilage biopsy (typically the intercondylar notch). Perhaps the most striking example that this may be a problem is in a study of ACI for lesions of the talus, where a biopsy was taken from an otherwise asymptomatic knee. Seven out of 10 patients suffered from sustained, moderate knee pain 12 months after the biopsy (Whittaker, 2005; Matricali, 2010).

\section{Stem cell implantation}

As will be discussed further below, using MSCs is a promising way to eliminate the need for a cartilage biopsy. This has to some extent already been tried in several animal trials with or without scaffolds (Wakitani, 1994; Solchaga, 1999; Solchaga, 2002; Koga, 2007; Loken, 2008) (for review, see (Ringe, 2012; Tang, 2012)). The results are promising, but superiority to other treatments in animal studies is not consistent. In human trials, MSCs have only been used in few and limited case series, embedded in a type I collagen gel (Wakitani, 2002; Wakitani, 2004; Kuroda, 2007; Matsumoto, 2010). In the largest of these studies ( $n=12$ in each group), the bone marrow-derived MSCs were used to treat osteoarthritic knees after high tibial osteotomy. Cells were implanted under periosteal flaps and compared to cell free implants. No clinical difference was observed, but the repair tissue in the cell group scored higher arthroscopically and histologically (partially 
hyaline cartilage-like). Although these studies do provide proof of principle, they can only serve as foundations for a more stringently designed study that is adequately powered to show clinically significant differences or non-inferiority. As will be discussed below, the safety of using stem cells is an issue. These studies in combination with animal studies provide substantial evidence of the safety of these procedures, as no tumour growth has been seen. 


\section{Stem cell biology}

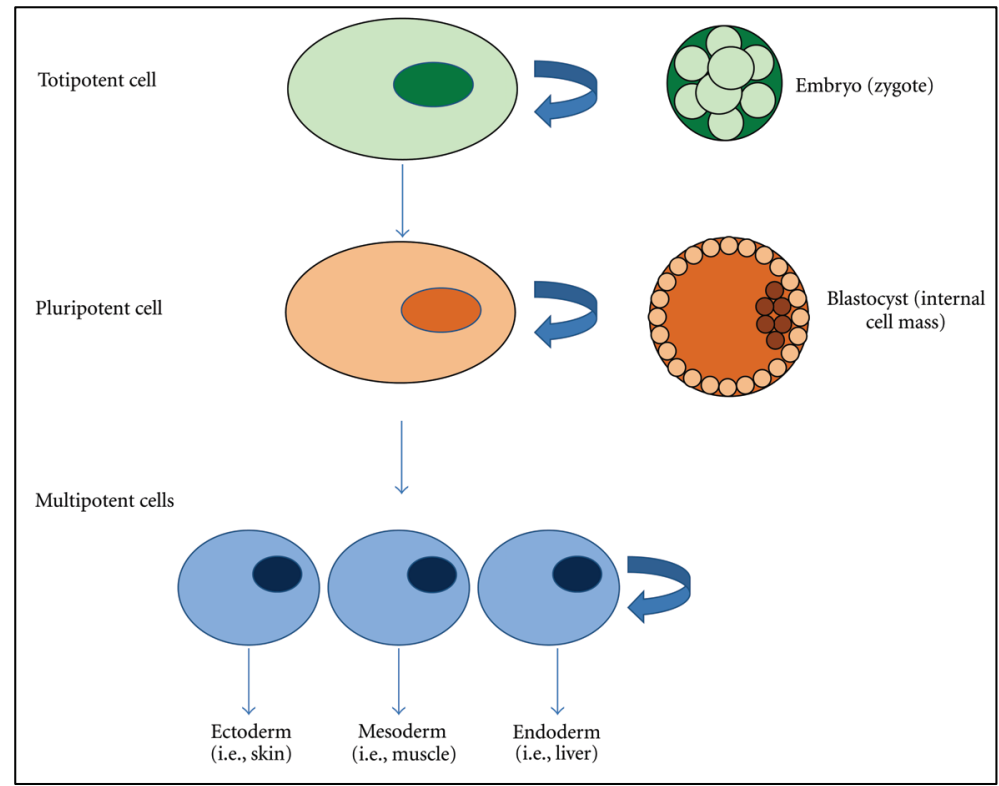

Figure 5 Schematic representation of stem cell properties. Adapted from (Diez

Villanueva, 2012).

Stem cells are defined as cells that throughout the lifetime of an organism are able to give rise to new stem cells (a property termed self-renewal) and to the variety of differentiated cells within that organism (a property termed potency) (Weissman, 2000; Morrison and Kimble, 2006).

\section{Stem cell nomenclature and definitions}

Stem cells are classified by their potency and origin (Figure 5). At the top of the hierarchy is the totipotent stem cell, which is able to give rise to all cells in a developing embryo, including the extraembryonic tissues. The only known totipotent stem cell is derived directly from the fertilised zygote and can be obtained until approximately day five after fertilisation (i.e., the first cell divisions and the morula stage), when the blastocyst forms (Sadler, 2006). From there on, totipotency is lost and cells derived from the inner cell mass of the blastocyst are 
termed pluripotent and are able to differentiate into all embryonic tissues. These cells are the quintessential stem cells referred to as embryonic stem cells (ESCs). Human ESCs were first isolated and established in 1998 (Thomson, 1998).

Yamanaka and coworkers discovered another source of pluripotent stem cells (Takahashi, 2006). They found that overexpression of only four gene products (OCT4, SOX2, KLF4 and cMYC) in fully differentiated skin fibroblasts prompted a tiny proportion of these cells to become fully pluripotent (induced pluripotent stem cells - iPS cells). This work was quickly expanded on by the same and other groups and has become an extremely valuable tool in molecular biology (Okita, 2007; Yu, 2007). It is used to study rare genetic diseases, to screen for new drugs and to understand stem cell differentiation (for review, see (Robinton, 2012)). In 2012 Yamanaka shared the Nobel Prize in Physiology or Medicine with the renowned developmental biologist John Gurdon. Although iPS cells become very similar to ESC there are significant differences in the epigenetic signature (Huang, 2013). Interestingly, it has also been shown that just the introduction of the microRNA cluster miR302/367 could induce pluripotency in human and mouse somatic cells without the so-called Yamanaka-factors (Anokye-Danso, 2011).

Somatic stem cells, also referred to as adult stem cells, reside in differentiated tissues and are thought to be responsible for the general homeostasis of cells in that particular tissue as well as participating in the repair after injury or disease (Joseph, 2005). They are restricted in potency, being able to differentiate into lineages specific to that tissue, and have been found in almost all tissues, including tissues previously thought not to regenerate, such as the central nervous system. Some somatic stem cells are multipotent, one example being the haematopoietic stem cells, which constantly repopulate the blood system with all cell lineages specific to haematopoiesis, while others are believed to be only unipotent, being able to differentiate into only one lineage (myosatellite cells of muscles, for 
example (Holterman, 2005)). In general (at least in vivo), somatic stem cells lack the ability to differentiate into cells of other tissues (Joseph, 2005).

MSCs are a subgroup of somatic stem cells. These multipotent cells were first identified in bone marrow from guinea pigs (Friedenstein, 1970) and subsequently isolated from a wide range of human tissues, including bone marrow (Pittenger, 1999; Jiang, 2002), adipose tissue (Zuk, 2001; Boquest, 2005; Boquest, 2006), skeletal muscle (Cao, 2004), trabecular bone (Chen, 2005), epidermis (Watt, 2006), peripheral blood (Zhao, 2003), synovium (De Bari, 2001), periosteum (De Bari, 2006), teeth (Yamazaki, 2007), amniotic fluid (Kaviani, 2001), placenta (Miao, 2006) and umbilical cord blood (Kogler, 2004) (for reviews, see (Chamberlain, 2007; Phinney, 2013)). The abundance of tissues from which MSCs have been isolated suggests that they may be present in almost all tissues, although both this and the actual origin of MSC in various tissues are debated. Also controversial is whether they are indeed multipotent or if they possess pluripotentiality, being able to differentiate into cells not only of mesenchymal origin but also into ecto- and endodermal tissue.

The actual differentiation of a stem cell happens through progenitor or precursor cells committed to differentiate into one or a few mature cell types. For this to happen, fulfilling the requirement for self-renewal, stem cells need to undergo asymmetric division, giving rise to one new stem cell and one committed progenitor cell. Stem cells also have the ability to symmetrically divide, giving rise to two new stem cells. The mechanisms for determining if division should be symmetric or asymmetric are not fully understood (Li, 2013).

\section{Somatic versus embryonic stem cells}

With a cell source able to differentiate into mature tissues, there is an obvious opportunity to try to develop clinical treatments for a variety of conditions, including bone healing, cartilage resurfacing, spinal cord injury, multiple sclerosis 
and diabetes. Whether to use somatic or embryonic/induced pluripotent cells is an ongoing debate with both ethical and practical arguments. ESCs are considered to have the greatest degree of differentiation potential, but as ESCs are obtained from fertilised eggs that are destroyed in the procedure, ethical considerations have been raised concerning their use. On more practical grounds, no viable way to overcome the need for immunosuppression to avoid rejection of transplanted ESCs has emerged. Also, a practical argument against the use of ESCs is the fact that they often spontaneously differentiate in vitro and frequently form teratomas in vivo (the latter, in fact, is considered an important marker of bona fide ESCs) (Findikli, 2006). Techniques have been proposed that overcome the practical obstacles of using ESCs in the clinical setting (Jaenisch, 2004; Solter, 2005; Hochedlinger, 2006; Jaenisch, 2006). However, the first human trial of ESCs to treat spinal cord injury was halted due to concerns about cyst formation and later abandoned by the company for financial reasons (Frantz, 2012). Another study using ESCs to treat age-related macular degeneration is ongoing (Schwartz, 2012). Using iPS cells tailor-made from the patient would bypass both the ethical and immunogenic concern, though the concern of tumour formation remains valid. The first human trial of iPS cells to treat age-related macular degeneration was initiated the summer of 2013 (Melville, 2013).

Somatic stem cells are not burdened with the same ethical considerations as ESCs. Several types of somatic stem cells are readily obtainable with little or no morbidity for the donor, and in a manner making them suitable for autologous transplantation. Indeed, haematopoietic stem cells have been used for decades in haematologic cancers, and several other clinical studies of MSCs have been performed (Horwitz, 2002; Wakitani, 2002; Baron, 2003; Le Blanc, 2004). To date, no tumour formation has been observed after human somatic stem cell transplantation in vivo, and the consensus in the field seems to be that within the normal ex vivo expansion period of 6-8 weeks, no tumorigenic properties are seen. 


\section{Mesenchymal stem/stromal cells}

\section{Definition, characterisation and niche}

As stated above, MSCs have been isolated from numerous tissues. Naturally, this has been achieved by the effort of several research groups, which in turn has led to ambiguities and inconsistencies both in isolation and expansion procedures, as well as in their defining characteristics. As a consequence, the Mesenchymal and Tissue Stem Cell Committee of The International Society for Cellular Therapy (ISCT) issued a position statement defining three minimal criteria for designation of multipotent mesenchymal stromal cells, thereby also proposing the term "stromal" to be used instead of "stem" (Horwitz, 2005; Dominici, 2006). First, the cells must adhere to plastic under standard culture conditions. Second, the cells should have a distinct and fairly homogenous phenotype, with $\geq 95 \%$ of the population expressing the cell surface markers CD105 (endoglin), CD73 (ecto5'nucleotidase) and CD90 (Thy-19). Additionally, $\leq 2 \%$ of the cells should express CD45 (a pan-leukocyte marker), CD34 (the endothelial and haematopoietic marker), CD14 or CD11 (both monocyte/macrophage markers), CD79 $\alpha$ or CD19 (both B-cell markers) or HLA-DR (a marker of professional antigen-presenting cells, only expressed on MSCs if stimulated by IFN- $\gamma$ ). Third, the cells must be able to differentiate into the osteogenic, the chondrogenic and the adipogenic lineages in vitro as assessed with standard staining methods. Karyotype stability of the cells throughout expansion should be considered but is not included in the criteria. Despite the high number of tissues from which MSCs can be isolated, little is actually known about their in vivo life. They are supposedly mostly quiescent, renewing themselves at a very slow rate. The term "stem cell niche" has been coined for the location within the tissue where the somatic stem cell resides, although for many tissues it is not known exactly where that may be (Moore, 2006). Interestingly, MSCs themselves seem to be critical for the niche supporting the haematopoietic stem cells (Frenette, 2013). The stem cell niche is defined as including all extracellular elements and other cells surrounding the stem cells in 
their native state, and it supposedly works to keep the stem cell in a quiescent and undifferentiated state (Lin, 2002). If the niche is broken or if signals from cytokines or other signalling molecules reach the stem cell, it is pushed into differentiation $(\mathrm{Li}, 2005)$. As somatic stem cells are few and far between compared to the numbers of differentiated cells in tissues, large difficulties arise when trying to investigate this niche directly in vivo. Maybe the most compelling indirect evidence of what characterises this niche for the MSCs comes from a study in mice, where cells isolated from brain, spleen, liver, kidney, thymus, lung, bone marrow, muscle, pancreas and the great vessels all showed MSC properties with a strikingly similar morphology, immunophenotype and to some extent differentiation potential (da Silva Meirelles, 2006), not unlike human MSCs. The authors hypothesised, in line with a few other papers that have investigated the niche (Doherty, 1998; Bianco, 2001; Shi, 2003), that the stem cells are intimately related to pericytes existing in a perivascular niche, close to the basement membrane of blood vessels. This was shown to be true in another study, but it was also shown that the pericyte contribution to the MSC population varied from tissue to tissue (Feng, 2011). The perivascular localisation would give MSCs easy access to most tissues for repair if needed (Francois, 2006). Although MSCs are present in peripheral blood, the difficulty of isolating them from this source does somewhat contradict this hypothesis.

\section{Differentiation potential and plasticity}

Plasticity of stem cells is a somewhat vague term with no clear consensus definition; it blends in with the property pluripotency described above. The following set of criteria for true plasticity have been suggested: A single stem cell should be able to self-renew, give rise to the functional cell types of the tissue from which it derives, give rise to cell types of unrelated tissues, and repopulate both tissues robustly in vivo (Serafini, 2006). The last criterion is the most difficult one to prove for any given cell, and often plasticity is proposed only on the basis of the first three (in essence showing in vitro pluripotency), and even 
then not always meeting the single-cell criterion (opening up the possibility of different cell types in the investigated population giving rise to the different cell types) (Wagers, 2004; Lakshmipathy, 2005). MSCs by definition should be able to differentiate into the adipogenic, osteogenic and chondrogenic lineages, but this is multipotency, not plasticity, as all three of these lineages are derived from the definitive mesoderm. Especially for both MSCs from bone marrow (BM-MSCs) and from adipose tissue (AT-MSCs), the differentiation criterion has been met in numerous studies. Evidence is sparser when it comes to plasticity. AT-MSCs have been shown to differentiate into neuron-like cells in clonal assays (Boquest, 2005; Guilak, 2006), into endothelial cells in non-clonal assays (Cao, 2005), into hepatocytes in non-clonal assays (Seo, 2005) and into epithelial cells in non-clonal assays (Brzoska, 2005), indicating pluripotentiality, but functionality in vivo has not been shown. Regarding BM-MSCs, the picture is even more clouded, yet also more promising (Serafini, 2006). Several populations of cells that show multi- and pluripotentiality have been isolated from the bone marrow by slightly different methods. All populations are adherent cells that exhibit many of the characteristics of MSCs, but also characteristics normally associated with ESCs.

The first population described was the multipotent adult progenitor cell (MAPC) (Reyes, 2001), from adherent bone marrow cultures supplemented with epidermal growth factor and platelet-derived growth factor and with a low serum concentration. They showed differentiation into multiple mesodermal lineages in clonal assays. Subsequently, in the mouse model, similar cells showed differentiation into tissues of all three germ layers at the single-cell level (Jiang, 2003). MAPCs additionally expressed the ESC-specific transcription factor Oct $3 / 4$ and were able to contribute to chimeras when injected into a blastocyst. Finally, when injected into non-damaged recipients, they showed robust engraftment in several tissues (Jiang, 2002). However, some concerns, particularly about the chimera experiments, have been raised by other researchers, as these results have 
proved hard to reproduce (NatureNews, 2007). Another cell population termed marrow-isolated adult multilineage inducible (MIAMI) cells are isolated and cultured using procedures almost identical to MAPCs and show similar differentiation potential into cell lineages of all three germ layers, although not at the single-cell level (D'Ippolito, 2004). One publication also showed the ability of these cells to differentiate into cells with electrophysiological properties similar to neurons (Tatard, 2007). A third population, termed human bone marrow-derived multipotent stem cells (Yoon, 2005), are similar but slightly different in phenotype from MAPC and MIAMI cells. The small differences seen in phenotypic appearance between all these populations may in fact be attributed to slightly different isolation and culture conditions. The debate around a cell type termed very small embryonic-like stem cells, a putative pluripotent tiny cell type present in bone marrow and other tissues, highlights some controversy regarding these subpopulations of stem cells (Kucia, 2006). Several groups have published that this cell type does indeed exist in humans, while other highly renowned groups refute their existence (Abbott, 2013; Miyanishi, 2013).

To date, no one has shown true plasticity of somatic stem cells in vivo before ex vivo expansion. On the other hand, the studies described above do show that ex vivo-expanded stem cells can give rise to functional cells after transplantation into recipients in vivo, and this alone gives them a possible therapeutic potential. What is not known is whether these cells exist as pluripotent cells in vivo or whether the ex vivo manipulation pushes the cells into a dedifferentiated state, thereby achieving pluripotentiality (Wagers, 2004). The knowledge from the iPS cell field supports to some extent the latter scenario, as a seemingly small change in the expression of a few factors can thoroughly change a cell state.

Objections to the pluripotentiality of somatic stem cells in vivo after ex vivo manipulation are many, one of which has been mentioned: the possibility that 
within the population isolated, more than one type of stem cell exists. No matter how purified the population of stem cells, no one has achieved $100 \%$ homogenous populations. Therefore, only in studies showing pluripotency of single-cell clones can plasticity really be proven. The phenomenon known as fusion is another objection raised by several authors against studies that have apparently shown functional cell engraftment in tissues after stem cell transplantation. Fusion was first seen in in vitro cocultures where ESCs adopted the phenotype of neural progenitors or bone marrow cells by fusion with these cells at a low rate (Terada, 2002; Ying, 2002). This has also been shown at very low rates in some in vivo systems (Medvinsky, 2003; Alvarez-Dolado, 2007), but in other studies it has been ruled out (Harris, 2004). Fusion may still be desirable if it aids in repairing injured tissue into functional tissue.

\section{Other applications of MSCs}

Beside the potential use of MSCs in tissue engineering, which is the focus of this thesis, another intriguing use of MSCs is in preventing or treating severe graftversus-host disease. Allogeneic MSCs are regarded as immunoprivileged because they avoid immune recognition and exert an immunosuppressive effect by secreting soluble factors (indoleamine 2,3-dioxygenase, nitric oxide and prostaglandin E2) and through direct cell-to-cell contact (English, 2013). Promising results have been shown in clinical trials, and multiple trials are ongoing (Le Blanc, 2004; Ryan, 2005; Ball, 2013). These findings have even led Arthur I. Caplan, who originally suggested the term MSC (Caplan, 1991), to suggest they be renamed medicinal stromal cells (Caplan, 2010).

\section{In vitro chondrogenesis of stem cells}

One of the key features of MSCs is the ability to differentiate into mesodermal lineages, such as the chondrogenic lineage. Different strategies have been used to 
induce this process, including the use of cytokines, hormonal stimuli and threedimensional culture methods.

\section{Pellet cultures with growth factors}

The basis for the pellet culture system came from the finding that pellet culture could prevent the dedifferentiation that occurred when chondrocytes were grown in monolayer cultures (Holtzer, 1960; Manning, 1967). This system was later refined for the chondrogenic induction of bone marrow (Johnstone, 1998). After centrifugation of $2 \times 10^{5}$ cells into a pellet, the pellet was cultured in a defined induction medium that contained transforming growth factor $\beta 1$ (TGF $\beta 1$ ) and dexamethasone. This treatment induced chondrogenesis in all pellet cultures, as evidenced by upregulated type II collagen production. Later, this induction medium was refined by adding different types of bone morphogenetic proteins (BMPs), which further enhanced chondrogenesis (Sekiya, 2001; Sekiya, 2005). In these first studies, it is rather unclear why the authors chose the added growth factors, but TGF $\beta 1$ and BMP-6, -2 and -4 belong to the TGF $\beta$ superfamily. This superfamily, which also contains activins and inhibins, Müllerian-inhibiting substance, cartilage-derived morphogenetic proteins, decapentaplegic gene product, Vgr gene products and glial cell line-derived neurotrophic factor, has very diverse effects in a wide range of tissues. These effects vary depending on the cell type and include effects on cell growth, proliferation, apoptosis, gene expression, differentiation and motility (for review, see (Kanaan, 2006; Derynck, 2007)). The complete function of TGF $\beta$ is not fully understood, but various members of the superfamily regulate key events in embryogenesis, including the forming of the primitive streak and the differentiation of mesoderm and ectoderm (Yang, 2003; Sadler, 2006).

The TGF $\beta$ subfamily in humans consists of three slightly different proteins $(\beta 1,-2$, $-3)$, the most studied of which is TGF $\beta 1$. All of these proteins are encoded as large protein precursors of approximately 400 amino acids, the 112-114 amino acid C- 
terminal region of which becomes the mature molecule that subsequently dimerizes into a $25-\mathrm{kDa}$ active molecule (Seibel, 2006). This dimer elicits its biological function mainly through two specific transmembrane receptors, TGBR1 and TGFBR2. The receptor-ligand complexes in turn work through an intracellular signalling cascade of specific proteins known as the SMAD family of mediators of TGF $\beta$ signalling (Derynck, 1996). The end effector of this cascade is an activated complex of SMAD4 and SMAD2/3 proteins that, after translocation into the nucleus, forms a final complex with a large variety of cofactors. Finally, the complex acts as a gene transcription factor generating approximately 300 early gene responses (Massague, 2006). The SMAD pathway, from ligand binding to phosphorylation of intracellular SMAD proteins, is rather simple compared to other pathways, although the responses observed in different cell types are very heterogeneous. The discovery that a large variety of cofactors form complexes with the activated SMAD complexes is believed to be the explanation for the different responses of TGF $\beta$ in different cell types (Feng, 2005). Functionally, BMPs work in a similar way through specific receptors that activate the SMAD cascade using primarily SMAD- $1,-5$, and -8 . They ultimately elicit their function as transcription factors that are of activating, enhancing, or inhibiting nature, often in conjunction with transcription factors already present in the nucleus (Seibel, 2006; Keller, 2011). BMPs, like TGF $\beta$ proteins, are synthesised as precursors and, after proteolytic cleavage, form a biologically active dimer. More than 20 different BMPs have been described to have large and varied effects on most cells in general and especially on stem cells (Wagner, 2007; Retting, 2009). Briefly, BMP$2,-4$, and -6 have been shown to directly induce SOX9 expression, which is important for type II collagen production (Shum, 2002; Sekiya, 2005). BMP-2, -4, -5 , and -7 can all induce new bone formation in vivo but are also frequently used in in vitro chondrogenesis (Handorf, 2014). Finally, several BMPs have been shown to have profound effects on differentiation into mesenchymal lineages of 
stem cells, although a consensus on what is the most efficient dose and mix of BMPs for a specific differentiation of a specific cell type is far from being reached.

In summary, BMPs and TGFs have profound effects on cells during embryogenesis, especially on the skeleton and associated structures, and, not surprisingly, have profound effects on stem cells in vitro. The effects depend on the type of BMP/TGF protein and their mix and dosing and on cell type.

Several other molecules have been used in attempts to improve chondrogenesis. These include fibroblast growth factors, parathyroid hormone-related peptide and various inhibitors of signalling cascades (Liu, 2007; Kim, 2008; Kim, 2010b; Weiss, 2010; Handorf, 2011; Im, 2011). Some have been shown to lead to improvement, but there does not seem to be any consensus among research groups that these molecules should be included in the standard cocktails used to induce chondrogenesis. This debate might change in the coming years.

\section{Matrix-assisted cultures}

As discussed above, a three-dimensional culture of chondrocytes or stem cells is a requirement for chondrogenesis. Pellet cultures are, however, not a feasible strategy for tissue engineering, as they cannot be made a sufficient size without suffering from central necrosis due to limited oxygen and nutrient passage. Therefore, extensive research has been focused on finding a scaffold that can be used for in vitro studies of chondrocytes and MSCs (Hunziker, 2002a; Ge, 2012). The ideal scaffold would support cell viability, distribution, and proliferation; be biocompatible and biodegradable; provide structural support; and have a proper pore size to allow for diffusion of nutrients and waste products. For clinical use, they should also be suitable for handling and should be able to allow for integration to the adjacent tissue. Scaffolds used in the literature have been protein-based, made from fibrin, collagen and gelatin, or carbohydrate-based polymers, such as alginate, polylactic acid, polyglycolic acid and hyaluronic acid 
scaffolds. In addition, artificial polymers such as Dacron, Teflon, hydroxyapatite, titanium and carbon fibres have been used. Some of these scaffolds, in addition to capturing the cells in a three-dimensional system, provide biological cues to the cells via cell-matrix interactions. These are typically scaffolds made from constituents normally present in the ECM, such as collagen and hyaluronic acids. Other scaffolds, such as alginate derived from brown algae and polylactic and polyglycolic acid, are rather inert and do not bind to cell receptors.

Currently, no scaffold has emerged as unequivocally superior (Ge, 2012). It seems that MSCs and chondrocytes can undergo chondrogenesis in a large variety of scaffolds. However, a major problem for most, if not all, systems is that the differentiated cells do not stop in the prehypertrophic state. They continue to express the unwanted type I collagen, and induce type X collagen expression, as well as unwanted enzymes MMP13 and alkaline phosphatase and osteogenic transcription factor RUNX2 (Pelttari, 2006; Dickhut, 2009; Tang, 2012). The work in this thesis is based on scaffolds made from alginate or hyaluronic acid. These types of systems have been used successfully by several other research groups (Ma, 2003; Cristino, 2005; Tognana, 2007; Gharravi, 2012; Ma, 2012). 


\section{MicroRNAs}

MicroRNAs are a fairly recently discovered class of non-coding RNAs that regulate gene and protein expression (for reviews, see (Ambros, 2004; Bartel, 2004, 2009)). MicroRNAs are double-stranded and 21-25 nucleotides long when fully processed. They target complementary nucleotide sequences in mRNA,

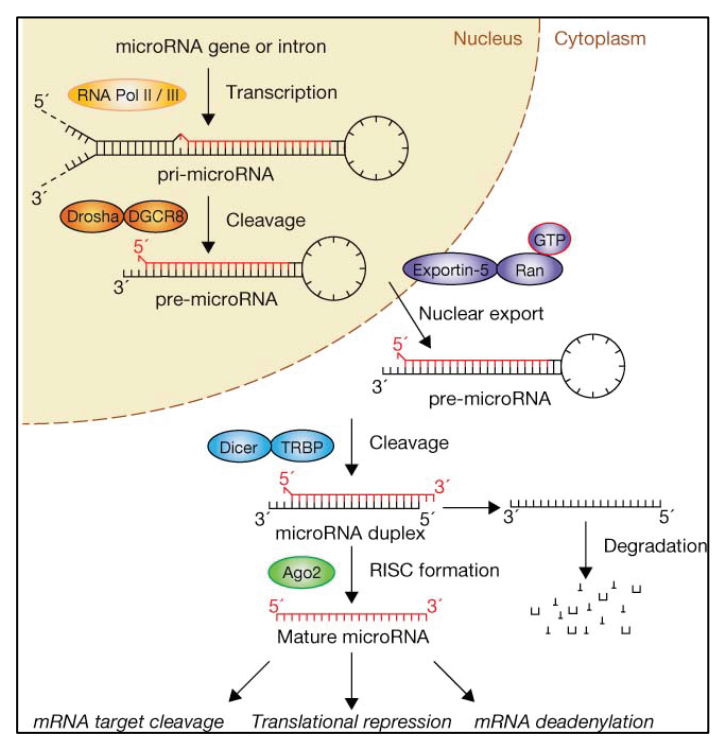

Figure 6. Canonical maturation of mammalian microRNAs. See text for explanation. From (Winter, 2009) primarily in the 3 - -untranslated region (3'UTR) in animals

(Figure 6). They are transcribed as longer transcripts, termed primary microRNA. Primary microRNAs are recognised by the Drosha microprocessor complex in the nucleus and are cleaved, leaving a 3' twonucleotide overhang hairpin precursor microRNA. The precursor is transported via exportin 5 to the cytoplasm,

where it is processed by Dicer, which cleaves the hairpin loop, leaving the mature double-stranded microRNA. One or both of the strands may have a biological effect (in general based on the stability of each strand) and are given suffixes based on which arm they come from (the $5 p$ or $3 p$ arm). Mature microRNAs are then incorporated into the RNA-induced silencing complex (RISC) and may then exert their effect by binding with full or incomplete complementarity to mRNA (He, 2004). Regarding complementarity, one normally considers only the so-called seed sequence, which is the sequence from nucleotide two to nucleotide seven counted from the $5^{\prime}$ end, though it is sometimes expanded to nucleotides eight and 
one (Bartel, 2009). Full complementarity of this sequence generally leads to cleavage of the mRNA, which is then degraded. Incomplete complementarity generally leads to translational repression so that gene products are not produced; however, the mRNA is unaffected. New discoveries have also expanded our view of how microRNAs work by showing that they may, in some instances, enhance gene expression through activation instead of translation or by binding directly to promoters inducing or repressing transcription (Lin, 2011; Vasudevan, 2012; Meijer, 2013). Binding may also occur in the 5'UTR (Orom, 2008).

The latest release of the full catalogue of all known microRNAs maintained by the University of Manchester (www.mirbase.org) lists more than 2,000 human microRNAs. As one microRNA may target more than 100 different mRNAs, the total effect of microRNAs in human cells is mind-boggling (Brennecke, 2005). It is estimated that $60 \%$ of all genes are regulated by microRNAs to some extent (Friedman, 2009).

\section{Role of microRNAs in cartilage biology and pathology}

The essential role of microRNAs in the normal development of cartilage was convincingly demonstrated in mice deficient for Dicer in chondrogenic regions. These mice showed severe skeletal malformations and died in early postnatal life (Kobayashi, 2008). One specific microRNA, miR-140, has been identified as especially important in cartilage and is almost exclusively expressed in cartilage (Tuddenham, 2006; Miyaki, 2009; Araldi, 2010; Miyaki, 2010). miR-140 is located in an intron in the WWP2 gene and has its own promoter; its expression is induced by SOX9 (Yang, 2011b; Nakamura, 2012). In vivo studies have substantiated the importance of miR-140 in chondrogenesis. miR-140-deficient mice show relatively small skeletal differences compared to wild-type mice. The skeleton of miR-140-deficient mice is slightly shorter, and the cartilage growth plate is thinner with fewer proliferating chondrocytes compared to wild-type mice. More strikingly, the deficient mice were much more prone to develop both age- 
related and surgically induced osteoarthritis. The explanation for the susceptibility to osteoarthritis might be that miR-140 targets the aggrecanase ADAMTS5 (Miyaki, 2010). This corresponds with the finding that miR-140 expression is reduced in human osteoarthritic cartilage in which a loss of proteoglycans is often a hallmark (Iliopoulos, 2008; Miyaki, 2009). It has also been shown that miR-140 deficiency leads to accelerated hypertrophic differentiation of chondrocytes, thereby impairing endochondral bone formation, possibly by modulating BMP signalling through the target DNPEP gene (Nakamura, 2011).

Multiple publications support the significance of miR-140 in chondrogenesis. This microRNA has been validated to target SMAD3 (only at the protein level), SP1, $B M P 2, C X C L 12$ and $H D A C 4$, which are all known to influence cartilage development or homeostasis (Tuddenham, 2006; Nicolas, 2008; Pais, 2010; Nicolas, 2011b; Yang, 2011b).

It is likely that other microRNAs play important roles in cartilage development. For example, miR-145 has been shown to target SOX9 (Yang, 2011a; MartinezSanchez, 2012). miR-675, if overexpressed, positively regulates type II collagen expression in chondrocytes (Dudek, 2010). The bicistronic miR-family of miR221 and miR-222 has been shown to negatively regulate chondrogenesis in chick limb stem cells, possibly through an influence on mechanotransduction (Dunn, 2009; Kim, 2010a).

In osteoarthritic cartilage, a variety of microRNAs has been shown to be dysregulated. For example, the expression of miR-25, -26a, -27a, -27, -29a, -140, 210 , and -337 is lower in osteoarthritic cartilage compared to normal cartilage. The significance of these changes remains to be determined for most of the microRNAs, with miR-140 as a notable exception (Goldring, 2006a; Iliopoulos, 2008; Miyaki, 2012). 


\section{Future applications of microRNA in treatment, diagnosis, and prognosis}

As illustrated by miR-140 and osteoarthritis, microRNAs may be dysregulated in human disease. Many other examples of microRNA dysregulation have been found in recent years, especially within cancer and autoimmune diseases (Thai, 2010). However, in many instances, it is not known whether the dysregulation is causal. Nevertheless, some of these dysregulations are quite specific and may therefore be potential biomarkers for diagnosis and prognosis. For example, miR21 has been proposed as a marker for colorectal cancer, miR-221/222 as a marker to aid in prognosis in advanced breast cancers, and miR-30d has been investigated as a prognostic marker in prostate cancer (Kobayashi, 2012; Falkenberg, 2013; Toiyama, 2013). These types of studies have mainly been conducted on tissue samples, but recent studies have also tried to use circulating microRNAs isolated from serum or plasma as a non-invasive opportunity to access disease-specific information (Ouyang, 2013). The exact origin or function of circulating microRNAs are not entirely clear, although it appears that cells secrete microRNAs continuously, normally as exosomes, and in some instances as free

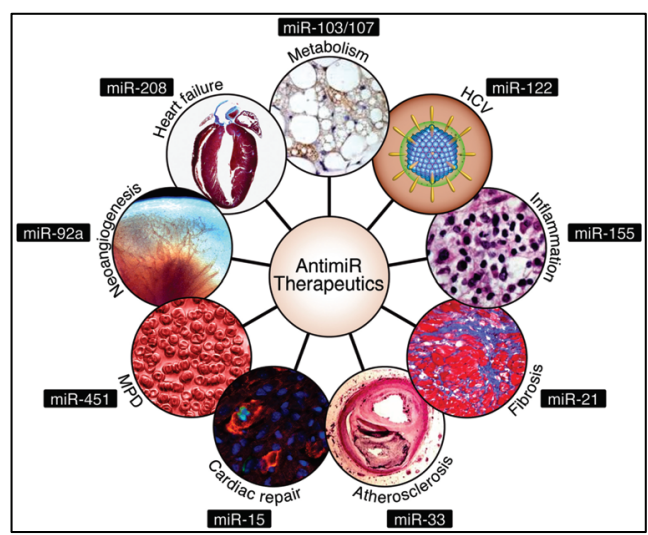

Figure 7 A subset of microRNAs currently being pursued in preclinical and clinical trials (MPD: myeloproliferate diseases; HCV: hepatitis $\mathrm{C}$ virus). From (van Rooij, 2012).
microRNAs that circulate bound to RNA-binding proteins.

Additionally, it not entirely clear if this secretion is a representative sample of the cytoplasmic microRNAs or if the mechanism has some level of selectivity (De Guire, 2013). It is likely that the research field on circulating microRNAs as disease markers will expand considerably in the coming years. 
MicroRNAs are also being investigated for therapeutic purposes. A novel example of this is the use of a miR-122 inhibitor as a treatment for hepatitis $\mathrm{C}$ virus infection. This virus type is dependent on miR-122 expression for replication (Lanford, 2010). In a phase 2a clinical trial, a specific inhibitor of miR-122 (Miravirsen ${ }^{\circledR}$ ) was shown to induce a dose-dependent reduction in hepatitis $\mathrm{C}$ virus RNA without evidence of viral resistance (Janssen, 2013). Other anti-miRs being pursued for therapy are illustrated in Figure 7.

So far, there have not been clinical trials of microRNA mimics for therapeutic purposes, although there are some preclinical studies with promising results. Let$7 \mathrm{~b}$ mimics were shown to reduce tumour formation in a mouse model of lung cancer (Esquela-Kerscher, 2008). Recently, a phase 1 clinical trial was announced to test a mimic of miR-34, believed to act as a tumour suppressor in patients with unresectable primary liver cancer (http://clinicaltrials.gov/ct2/show/NCT01829971). More related to the subject of this thesis, intra-articular injection of miR-210 was shown to be effective in promoting healing of torn ACLs in a rat model (Shoji, 2012).

Several companies have been established to research a multitude of microRNAs and anti-microRNAs the last few years, and it is likely that new therapies will evolve in the coming decade. 


\section{Aims of the study}

The overall aim of this study was to explore the biology of in vitro chondrogenesis in a translational setting. We aimed to deepen the existing knowledge on several aspects of tissue engineering of human articular cartilage as described below.

Specific aims related to the included papers:

Paper I. To examine the in vitro chondrogenic potential of adipose tissue-derived and bone marrow-derived MSCs in a clinically available scaffold made from esterified hyaluronic acid, as evaluated by gene and protein expression of key cartilage markers. Dedifferentiated chondrocytes cultured in the same scaffold were used for comparison.

Paper II. To evaluate if MSCs could be harvested, cultured and implanted in the same scaffold as used above in paper I for the repair of a critically sized osteochondral defect in a rabbit model.

Paper III. To investigate the global microRNA expression profile of human MSCs undergoing chondrogenic differentiation and compare the profile to that of freshly isolated human articular chondrocytes undergoing dedifferentiation to identify those with a reciprocal relationship. Subsequently, to investigate the role of these microRNAs in in vitro chondrogenesis and identify possible targets.

Paper IV. To validate the use of factorial design and high-throughput mRNA profiling to dissect in vitro chondrogenesis in general; specifically, to analyse the effects of five commonly used chondrogenic factors in directed differentiation of human bone marrow-derived MSCs. 


\section{Summary of results}

\section{Paper I}

In this study, we compared MSCs from adipose tissue and bone marrow with articular chondrocytes. We used a clinically available scaffold made from nonwoven hyaluronic acid, HYAFF-11®, which at the time of our experiments was being tested in clinical trials with cultured chondrocytes for the repair of chondral defects. Cells were expanded in monolayer cultures and seeded onto the scaffold. Three-dimensional MSC cultures were supplemented with a chondrogenic medium containing TGF $\beta 1$, BMP2 and dexamethasone. Three-dimensional chondrocyte cultures were not given chondrogenic medium to keep the culture conditions similar to what was used in the clinical trials. We assessed chondrogenesis using quantitative RT-PCR and immunohistochemistry on fixed sections and by ELISA on supernatants.

We found that the MSC cultures responded to the medium and scaffold by upregulating chondrogenic markers measured at the mRNA level. Notably, this included both the upregulation of the wanted hyaline cartilage marker COL2A1 and potentially unwanted markers, such as COL10A1. The most pronounced effect was observed when the MSCs were continuously exposed to chondrogenic factors. Bone marrow-derived MSCs induced much higher levels of type II collagen mRNA expression than did adipose tissue-derived MSCs and chondrocytes. Type II collagen protein was seen by immunohistochemistry within the cells in the scaffold; however, only small amounts of extracellular type II collagen were observed extracellularly. Instead, considerable amounts of type II collagen were found in the culture supernatants. 


\section{Paper II}

Although ACI is widely used, there are concerns related to its use, especially donor site morbidity, the need for a two-stage intra-articular procedure and the risk of graft hypertrophy. In addition, the dedifferentiation of chondrocytes in culture questions whether the chondrocytes will be able to re-establish good quality hyaline cartilage. The use of MSCs as an alternative would effectively eliminate these concerns. Therefore, the purpose of this study was to evaluate the efficiency of MSCs in a hyaluronic acid scaffold to repair an osteochondral defect in rabbit knee. We harvested bone marrow from 11 New Zealand White rabbits, isolated MSCs and cultured them in autologous serum for 28 days. Forty-eight hours prior to implantation, cells were seeded onto the scaffold at density of $10^{7}$ cells $/ \mathrm{cm}^{2}$. Circular defects that were $4 \mathrm{~mm}$ wide and $1.5 \mathrm{~mm}$ deep were created on the medial femoral condyle of both knees, and the cell-loaded scaffold was implanted in one knee and an empty scaffold in the other knee. The rabbits were killed after 24 weeks. Condyles were fixed and subjected to semiquantitative and quantitative evaluation by blinded observers. We found a high degree of filling in both cellloaded and control defects, with no statistically significant difference in filling. However, there was a tendency for better quality repair tissue in the cell-treated defects. We did not observe any hypertrophy in either group.

\section{Paper III}

In paper III, we focused on the biology of in vitro chondrogenesis of MSCs compared to the dedifferentiation process of articular chondrocytes in culture. Based on a previous study from our group (Karlsen, 2011), we particularly wanted to study the microRNA expression profile during in vitro chondrogenesis in a novel self-gelling alginate scaffold. We first identified cartilage-related miR-140$5 p$ and $-3 p$ to be among the most highly expressed microRNAs in differentiated MSCs. We also identified miR-210 and miR-221 to be reciprocally expressed. 
miR-210 increased during differentiation of MSCs and decreased during dedifferentiation of chondrocytes, whereas the opposite was true for miR-221. There was a $57 \%$ overlap for the 100 most highly expressed microRNAs in differentiated MSCs and uncultured chondrocytes; for other microRNAs, the expression pattern was quite different.

Next, we transiently and stably overexpressed or inhibited miR-140-5p and $-3 p$. Global mRNA analysis showed that inhibition of miR-140-5p led to the upregulation of genes involved in cytoskeleton remodelling and cell division and the downregulation of genes related to extracellular regions and ECM. Further, we found chondrogenesis to be functionally impaired in MSCs stably overexpressing anti-miR-140-5p as assessed by protein expression of key cartilage markers and secretion of GAGs. Surprisingly, SOX9 and aggrecan proteins were found to be downregulated in anti-miR-140-transduced differentiating MSCs despite unchanged mRNA levels. This suggests that miR-140 may directly or indirectly stimulate in vitro chondrogenesis by the upregulation of these molecules at the protein level. We identified several possible new targets of miR-140-5p and $-3 p$, including CD248, LIPA, RALA, THBS2, and TMEM119. We validated RALA to be a target by luciferase reporter assay. Knockdown experiments showed that RALA regulated SOX9 at the protein level. RALA is a small GTPase believed to be involved in TGF $\beta /$ activin signalling but not previously known to play a role in chondrogenesis.

\section{Paper IV}

The in vitro process of chondrogenic differentiation of MSCs for tissue engineering requires three-dimensional culture along with the addition of differentiation factors to the culture medium. In this study, we focused on the role of differentiation factors in the same alginate scaffold as used in paper III. 
Our aim was to explore factorial quality-by-design and high-throughput digital mRNA profiling as tools to characterise directed differentiation of MSCs and to validate the most commonly used chondrogenic growth factors. Factors used for chondrogenesis vary but regularly include members of the TGF $\beta$ superfamily and dexamethasone, sometimes in conjunction with fibroblast growth factor 2 and insulin-like growth factor 1 . We combined a factorial quality-by-design experiment with high-throughput mRNA profiling of a customised chondrogenesis-related gene set of 364 genes to study in vitro chondrogenesis of bone marrow-derived MSCs in alginate. Forty-eight different conditions of different combinations of TGF $\beta 1,-2$ and -3 ; BMP2, -4 and -6; dexamethasone; insulin-like growth factor 1; fibroblast growth factor 2 and cell seeding density were included in the experiment and assayed after one and seven days of induction in several donors.

First, we showed that high-throughput mRNA profiling could be efficiently performed on lysates of MSCs during in vitro chondrogenesis in alginate. We also found that changes in gene expression observed just one day after induction predicted later changes. The analysis revealed that the best of the tested differentiation cocktails included TGF $\beta 1$ and dexamethasone. Dexamethasone acted in synergy with TGF $\beta 1$ by increasing many chondrogenic markers while directly downregulating the expression of the pro-osteogenic gene osteocalcin. However, all factors beneficial to the expression of desirable hyaline cartilage markers also induced undesirable molecules, indicating that perfect chondrogenic differentiation could not be achieved with the current differentiation protocols.

The method validated in this study could quite easily be adapted to screen large numbers of molecules that might enhance chondrogenesis. Together, the implications of these findings could decrease both the cost and workload considerably in future experiments. 


\section{Methodological considerations}

In this section, a brief introduction is given to the methods used in the included papers highlighting some of the advantages and limitations. The section is not exhaustive. Methods that are very standardised and only used in a limited number or supplementary experiments are not discussed in detail. When relevant, problems that occurred during experiments and how they were solved or avoided will be mentioned. Detailed descriptions of the protocols used are in the methods and materials sections of the original papers.

\section{Cell culture and supplements}

Isolation of MSCs from bone marrow and adipose tissue was performed using established protocols previously described by our group and others and included a centrifugation step to separate out the mononucleated cells, which were then cultured in standard plastic culture flasks. Adipose tissue was obtained as a "byproduct" from elective liposuction procedures, and bone marrow was obtained by aspiration from the iliac crest of human volunteers. Articular chondrocytes were isolated from healthy or diseased cartilage biopsies using short-time enzymatic digestion before standard cell culture, following established protocols (Shahdadfar, 2008). All procedures on humans were approved by the Regional Committee for Medical Research Ethics and on rabbits by the Norwegian Animal Research Authority.

During monolayer culture, we used standard culture medium supplemented with antibiotics. Most cell types also need the medium to be supplemented with foetal bovine serum (FBS) for survival and proliferation. In the cultures in the later experiments included here, we substituted FBS with human platelet lysate plasma (hPLP) produced following published protocols (Schallmoser, 2009). This reflects a change in our lab over the last few years that happened for two main reasons: 
Firstly, we have made an effort to fully humanise our culture system to avoid any issues related to xenoreactivity, and secondly, FBS variability is a recognised fact that especially for MSC culture represents a problem in that different batches of FBS vary immensely in the ability to support MSC proliferation (Caplan, 2005).

\section{Three-dimensional culture and choice of scaffold}

In vitro chondrogenesis of stem cells requires a three-dimensional culture system. As presented in the introduction, a multitude of different systems and scaffolds have been used. In the work presented here, we used two different scaffolds, one made from hyaluronic acid (Hyaff-11®) and one made from alginate, which represent two different approaches. Hyaluronic acid is a constituent of the ECM of articular cartilage with a defined binding to and effect on chondrocytes via CD44. The Hyaff-11® scaffold is a polymer derived from the total esterification of sodium hyaluronate $(80-200 \mathrm{kDa})$. The configuration we used was a non-woven mesh composed of a random array of polymer fibres with a diameter of approximately $40 \mathrm{~nm}$. In aqueous solution, the material hydrates and undergoes degradation by spontaneous hydrolysis of the ester bonds. Further degradation in vivo may then be faciliated by the enzyme family of hyaluronidases. This process is quite rapid when no cells are seeded into the scaffold in vitro, and studies in rats aimed to evaluate the in vivo biodegradation of Hyaff-11® have shown that the material had mostly disappeared after approximately 4 months following implantation (Grigolo, 2002). The material is easily sterilised by $\gamma$-irradiation. The clear advantages of this scaffold are the proven track record with chondrocytes in clinical studies, including the ability to implant the scaffold arthroscopically, and its excellent biocompatibility. However, as illustrated in the results from paper I, there are problems in retaining the extracellular matrix in the scaffold. We were kindly provided the scaffold by the manufacturer Fidia Advanced Biopolymers for the studies in papers I and II. However, we were not able to secure an agreement on using the scaffold in future clinical studies. In parallel with these experiments, 
our group had started working with scaffolds made from alginate for MSC culture (Duggal, 2009). It has been shown that encapsulating chondrocytes in alginate supports the articular chondrocyte phenotype in long-term cultures and promotes the re-expression of cartilage specific genes in dedifferentiated chondrocytes (Bonaventure, 1994; Hauselmann, 1994). There have been several other studies using various types of alginate scaffold for in vitro chondrogenesis of MSC (Ma, 2003; Gharravi, 2012; Ma, 2012). Our group has validated a novel self-gelling alginate system for in vitro chondrogenesis (Herlofsen, 2011). Therefore, for the experiments in papers III and IV, we used this scaffold. Alginate is a polysaccharide naturally found in brown seaweed. It consists of $\beta$-D-mannuronic acid (M) and $\alpha$-L-guluronic acid (G) monomers that are randomly distributed (Smidsrod, 1990). Divalent cations interact with the G-blocks, causing the polysaccharides to form a gel. Alginate has several advantages in tissue engineering. It is biocompatible and is already used as a delivery vehicle for drugs and as dressing for thermal burn wounds (Tonnesen, 2002; Opasanon, 2010). Ultrapure clinical-grade alginates are also readily available, which prevent issues of toxicity and immunogenicity. In its pure form, especially with a G-block content above $50 \%$, alginate has been shown to not induce significant immune responses (Andersen, 2012), although some publications have shown that a higher M-block content does induce an innate immune reaction (Yang, 2009). Alginate is not degraded actively after implantation in living tissue but is believed to decay slowly. However, this potential issue in tissue engineering could be overcome by dissolving the alginate by chelating agents, such as EDTA or citrate, which bind divalent cations (usually $\mathrm{Ca}^{2+}$ or $\mathrm{Sr}^{2+}$ ). Ideally, this chelation could be conducted as soon as sufficient ECM has been produced to support the three-dimensional structure. Other molecules, growth factors or supplements may also easily be mixed into the alginate before gelling. 
In summary, both scaffolds used in this study could support chondrogenesis; however, due to availability for independent research and excellent properties for future adaptations, the alginate scaffold is currently our scaffold of choice.

\section{Transfections and transductions}

To study the effect of microRNAs and to inhibit specific mRNAs, we used two different methods to introduce the microRNAs, small inhibiting RNAs (siRNA) or complementary sequences to specific micro-RNAs (anti-miRs) into our target cells. One method was transient, the other permanent. The transient method is generally referred to as transfection and intentionally introduces nucleic acids into the cell cytoplasm without any incorporation into the genome of the cell. Transfection methods fall in two main groups: physical, such as electroporation or microinjection, and chemical, such the use of lipid carriers or salts (Kim, 2010c). In both transfection methods, the introduced nucleic acids are fairly rapidly diluted out due to cell division or degradation, and in general, the effect is lost in 3-5 days. Cell type, confluency at the time of transfection, cell cycle phase and stability of the delivered RNA are some of the factors influencing how well transfection works, in addition to the transfection method.

We used liposomal transfections and electroporation in our experiments, but the results obtained in the lab at the time of the experiments for paper III led us to use electroporation almost exclusively in the published paper. It was found that liposomal delivery, in which nucleic acids are contained in spontaneously formed small vesicles (liposomes), led to a significant upregulation of immune-related genes (Karlsen, 2013). This upregulation could potentially affect the results even when controlled for by appropriate negative controls. The same effect is not observed when using electroporation, in which nucleic acids are introduced into the cells following a short electric impulse, which transiently makes small pores in the cell membrane. It requires specialised equipment; however, it is easily 
performed and works well on a wide variety of cells. The manufacturer often provides specialised protocols for various cell types, but adaption for each specific cell type and workflow is still needed. We optimised our protocols with regards to cell number and ratio of serum to cell medium to optimise cell survival and found an optimum of $10^{6}$ cells and $20 \%$ FBS per electroporation.

Both an advantage and disadvantage of transfection is its transience. On the one hand, the cells are left quite unharmed by the transfection with no interference with the cell genome; on the other hand, if one is interested in long-term effects, serial transfections are needed. In the case of three-dimensional culture, this is not possible because electroporation requires cells to be in suspension, and liposomes will not penetrate the alginate due to charge and size.

To overcome this problem, we used a standard viral method termed transduction. By combining viral genes and an expression vector for the RNA sequence we want the cells to express, it is possible to stably affect the cells. We used a system based on lentivirus genes (for a review of methods, see (Durand, 2011)). Briefly, several plasmids containing the genes encoding all necessary proteins needed to make infection-competent viral particles are transfected into a so-called packaging cell line together with a plasmid encoding the RNA sequence to be expressed in the target cells. The packaging cell line then packages this expression vector into viral particles and secretes them into the cell medium, which is harvested. This medium may then be added to the target cell line, whereby particles infect the cells, and the expression vector is reversely transcribed into DNA, which is then integrated by the viral integrase randomly into the genome of the target cell. The integrated DNA incorporates a constitutive promoter, in our case the CMV promoter, that drives the expression of the transgene. The insertion also constitutively expresses an antibiotic resistance gene, in our case, to puromycin, to select the transduced cells. This is a very effective way of overexpressing or 
knocking down genes or microRNAs; however, it involves several steps that need optimisation and also includes some potential hazards. The latter is generally dealt with by using later-generation systems with all viral genes on separate plasmids; the former by pilot experiments to determine optimal transfections of the packaging cell line, viral titre, multiplicity of infection, concentrations of antibiotics for selections and optimism. Disadvantages to be aware of are the random nature of integration that may disrupt tumour suppressor genes and that the CMV promoter has been shown to be gradually inactivated in stem cells (Xia, 2007).

\section{Gene and microRNA expression analysis}

\section{Quantitative RT-PCR}

Quantitative RT-PCR (RT-qPCR) has been the mainstay of gene expression analysis of a single or a moderate number of genes over the last decade. It is highly specific, sensitive and reproducible and allows for an easy assessment of the input RNA of a specific gene if a few requirements are met. It can be done either as an absolute quantification or relative to a reference sample, the latter being the most frequently applied. First, RNA should be of a sufficient quality and purity, usually judged by spectrophotometry. Second, the amount of cDNA formed during the reverse transcription reaction should accurately reflect the input RNA. This is secured by allowing for an excess of reagents and assessing for inhibitors of transcription by preparing dilution series. Third, a stable internal reference gene (endogenous control gene) is needed for normalisation, and this is normally tested for in each set of experiments, choosing from a common set of possible control genes. In the experiments included here, we have found the commonly used GAPDH gene to perform well. Fourth, the target gene and internal reference gene should have similar amplification efficiencies, preferably above 90\%. We generally use TaqMan probes (Applied Biosystems), which are tested by the supplier to perform to this standard or above, and we have tested several to 
confirm this. There are disadvantages of RT-qPCR, especially that it is feasible to test only a limited number of genes for practical and economic reasons. In addition, the constant debate on which reference genes or combination of reference genes to use, especially if reporting on only minor fold differences, needs consideration.

Finally, the problem with all gene expression assays is that they do not necessarily reflect functional differences in the cell, i.e., changes in protein expression.

\section{Nanostring}

A new way of testing gene expression has emerged the last few years. This is

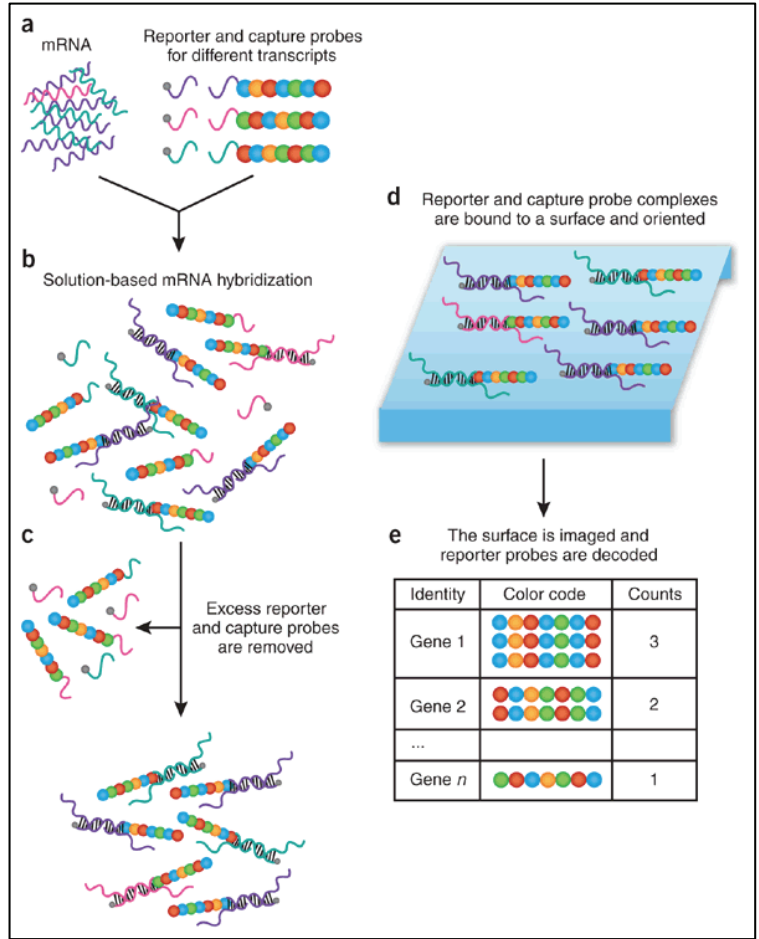

Figure 8 (a) Total RNA is mixed directly with nCounter reporter and capture probes. (b-d) After hybridisation (b), excess reporters and capture probes are removed (c), and the purified ternary complexes are bound to the imaging surface, elongated and immobilised (d). (e) Reporter probes, representing individual copies of mRNA, are tabulated for each gene. From (Fortina, 2008). marketed as Nanostring

nCounter Gene Expression, or in some publications, it is termed digital mRNA/microRNA profiling. We used this for gene and microRNA expression analysis in papers III and IV. The biggest difference from RTqPCR is that there are no enzymatic conversions or amplifications. The input RNA (purified or as cell lysates or even whole blood) is directly hybridised to fluorescently labelled probes (several fluorochromes are attached in a specific order to 
distinguish each probe from each other). These probes are subsequently immobilised in a cartridge, with a small current aligning all captured RNA in the same direction. Thereafter, the number of different probes is a direct measure of RNA templates captured (Geiss, 2008; Malkov, 2009).

The system has been shown to perform with sensitivity, specificity and reproducibility comparable to RT-qPCR but in a highly multiplexed system that can test up to 800 genes/microRNA in one sample. It has frequently been used to validate microarray analyses. The analyses of results are performed using measurements from spiked-in RNA to normalise for variability in hybridisation efficiency, and later using the same statistical analysis as applied for microarray data. Perhaps the biggest advantage of this method is that it can be performed directly on lysates of cells and tissues, allowing for a very efficient workflow in larger experiments and avoiding variability introduced by RNA isolation, although this does require a set of endogenous controls to be used to control for input.

\section{Functional assays (protein and proteoglycan quantification)}

\section{Western blots}

Western blotting, or, more accurately, protein blotting, is widely used to detect proteins in cell or tissue lysates, serum or cell culture supernatants. The technique involves both a size-separation phase by electrophoresis in a polymer membrane and a subsequent labelling of the blot with a specific antibody to the target protein. This so-called primary antibody is then bound by a secondary antibody that can be visualised by radioactivity, chemiluminescence, fluorescence, or gold particles, depending on the system used. In our system, we used a chemiluminescence approach. Generally, protein blotting is not used in a precise quantitative manner but rather in a semiquantitative manner judged "by eye" as showing more, less, or similar amounts of protein in the samples being compared. In any case, a judgement on the protein amount present depends on equal loading onto the 
membrane as determined by the loading control. The protein beta-actin is probably the most commonly used loading control. There are numerous steps involved in protein blotting, and each may be optimised for the specific cell type and antibody used. For our experiments, the step demanding the most optimisation was how to prepare good lysates from the alginate discs. We tried several ways, including dissolving the alginate by chelators or isolating protein by an adapted Trizol method, but these methods were unsuccessful. Instead, we ended up pulverising the alginate discs in liquid nitrogen and dissolving them directly in the lysis buffer before loading them onto the membranes. This worked well, but we did see broader bands in samples from alginate than from cell pellets, especially for aggrecan.

\section{ELISA}

Another way to quantify proteins using antibodies is the enzyme-linked immunosorbent assay used in paper I. This method uses a specific antibody to bind an antigen, in our case type II collagen. These assays are usually performed in special 96-well plates with commercially available kits, in which the secondary antibody is linked to an enzyme that, after adding of a specific substrate, produces a visible signal that can be read on a spectrophotometer.

\section{Immunohistochemistry}

A third way to visualise proteins is immunohistochemistry performed on sections of cells, tissues or, in our case, "tissue-engineered" cartilage. Whereas protein blotting gives a readout that is semiquantitative, immunohistochemistry on sections also gives a spatial readout. In our experiments, immunohistochemstry was used to validate the protein expression of cartilage markers. There are numerous optimisation steps needed for successful immunohistochemistry, which, in our case, was performed when the self-gelling alginate system was validated for chondrogenic differentiation (Herlofsen, 2011). 


\section{Quantification of sulphated proteoglycans and glycosaminoglycans}

The last method we employed to functionally support gene expression data, was to quantify the production of sulphated proteoglycans present in the culture medium. Much like for ELISA, we used a commercially available kit used by our group and others previously (Jia, 2012; Fernandes, 2013). This kit uses a specific dye that binds to sulphated GAGs and can be read on a standard spectrophotometer. Quantification is then performed with the help of a standard curve from supplied standard concentrations of GAGs.

\section{MicroRNA luciferase reporter assay}

To validate the binding of a microRNA to a specific mRNA, we utilised a luciferase reporter system (Nicolas, 2011a). This simple system uses the 3'UTR of the suspected target cloned into a vector constitutively expressing luciferase. If this vector is cotransfected with a microRNA that binds to this sequence, then the luciferase expression will be turned off due to cleavage or translational repression. Luciferase leads to light emission, which can be quantified on a plate reader. Any decrease in the level of emitted light suggests a binding of the microRNA. Combined with the appropriate controls of irrelevant miRs and 3'UTR-sequences, it is possible to validate that the microRNA directly affects the mRNA with a high degree of certainty. The vectors can be easily cloned in the lab, but to save time, we purchased a vector from a commercial vendor.

\section{Quality-by-design experiments}

Optimising a process one factor at a time is common in biological experiments. However, it is time consuming and laborious. In the chemical and other industries, factorial analysis, quality-by-design experiments, sometimes termed "Design of Experiment" approaches, have been utilised to determine the effect of several factors on a chosen response simultaneously in a cost-effective manner (Chen, 
2011b; Enochson, 2012; Crapnell, 2013). This demands a specific experimental set-up in which the investigated factors are included in different runs in a randomised manner. These set-ups can be easily designed with the use of

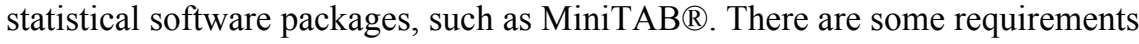
that need to be met for the analysis (which is essentially an analysis of variance (ANOVA)) to be valid, including that the data need to be tested for normality and, if needed, log-transformed to pass the normality test before testing for significant effects on the response. The data can then be fitted with a regression model, which will explain as much as possible of the variance in the dataset and subsequently test which factors affect the response significantly. More important, and a great advantage of this type of experimental setup, is that it is possible to test how factors influence each other in synergistic or antagonistic ways with regard to the chosen response. This technique potentially allows for a more efficient optimisation of processes than the standard one-factor-at-the-time-step-by-stepapproach.

\section{Animal experiments}

\section{The rabbit animal model}

Several animal models have been used in studies of cartilage repair, including sheep, goats, dogs, minipigs and horses (Hunziker, 2002). The rabbit model is by far the most commonly used, most likely due to size, availability, and price.

Rabbits have been used by our group for previous studies; therefore, this animal was chosen for the current study (Aroen et al., 2005; Aroen et al., 2006).

Advantages of using animal models are numerous; perhaps most importantly, the whole joint can be evaluated histologically as opposed to a small biopsy in human trials. All parameters other than the actual treatment can be held constant, which means that any observed changes are more likely be a cause of the treatment. Using an animal model also provides a way to observe the safety of a given treatment before initiating human trials. There are, however, several 
considerations to be aware of when using animal models. One is interspecies variations. In the rabbit, the height of the cartilage is approximately $0.4-1.0 \mathrm{~mm}$ as opposed to $2-3.3 \mathrm{~mm}$ in humans, and the subchondral bone of the rabbit is substantially thicker than in humans. This makes it very hard to make only grade 3 lesions experimentally, and most research has been performed on osteochondral lesions (Hunziker, 2002). In our experiments, we had a specialised burr made that allowed us to control the depth to $1.5 \mathrm{~mm}$ and $4 \mathrm{~mm}$ in diameter. It should also be considered that young animals have a very good natural healing response (Wei et al., 1997), stressing the fact that post-pubertal animals should be used, which for rabbits is when they are approximately 6 months and approximately $4 \mathrm{~kg}$ (O'Driscoll et al., 2001). Another consideration is that a seemingly large variation exists between so-called good healers and bad healers, which introduces a bias into the study unless large groups of animals are used or unless every animal is used as its own control (Solchaga et al., 1999). The latter is the preferred solution for most researchers. Bilateral lesions are made, and only one is given the experimental treatment. Finally, the issue of rehabilitation is hard to address in most animal models, especially smaller animals. We allowed rabbits to move freely immediately after surgery, which is commonly the norm but is significantly different from rehabilitation protocols in humans after cartilage surgery, in which early movement but no or low weight bearing activity seems to be the consensus (Mithoefer, 2012).

\section{Histology}

One of the advantages to studying cartilage repair in animal models is that the complete joint is available for evaluation when the research animals are killed. However, the three-dimensional lesion and repaired tissue are still only evaluated by a limited number of fixed and stained two-dimensional sections. In the rabbit study, we used two approaches to evaluate healing. First, a qualitative scoring, and second, a novel quantitative evaluation of filling based on point counting. 
The qualitative assay was modified from the original scoring system developed by O'Driscoll that evaluates five aspects of the repair tissue: 1. amount of hyaline cartilage, 2. surface regularity, 3. necrosis, 4. integration of borders, and 5. chondrocyte clustering. This or similar scoring systems have been used in numerous publications, but it does present some problems, for example, the importance of each parameter and its relation to the others is not clear. We therefore chose to report individual scores instead of a summarised score, as proposed in the ICRS scoring system (Mainil-Varlet, 2003). However, we also wanted to quantify filling by a more robust method, as the reproducibility and validity of the semiquantitative qualitative scoring systems have been questioned (Hyllested, 2002). We therefore used a computer program to overlay the sections randomly with a grid. A round of pilot experiments led to a grid with $200-\mu \mathrm{m}$ squares, resulting in approximately 160 points overlying the micrograph as shown in the paper. Every point was then scored as being above or below the tidemark and as bone, cartilage, or no tissue, allowing the proportions to be calculated. This method had excellent inter- and intra-rater reliability and was chosen as our main outcome.

There are several approaches to studying the histology of cartilage repair, and in recent years, more automated methods have been put forward. However, the recent white paper by the ICRS still advocates the semiquantitative qualitative scores and scores for the filling of the defects, but stresses the fact that evaluation should be blinded and performed by at least two independent investigators (Hoemann, 2011).

\section{Statistical considerations}

\section{Paper I}

The statistics in paper I are primarily related to the gene expression data. We used one-way analysis of variance (ANOVA) to compare the means of the gene 
expression levels within each donor type. Normally, if only one mean is compared to another, a Student's t test would be the standard parametric method, but as soon as more than two groups/time points are compared, the problem of multiple comparison and how to correct for it arises. In our case, ANOVA was coupled with Tukey's post-hoc test, which compares every mean to every other mean, and we report a p-value adjusted for the multiple testing. There are some assumptions for an ANOVA test to be valid: 1. The populations should have approximately the same variance, though this assumption is not very important when groups have the same number of subjects. The variance can be tested using the Bartlett's test, which is performed automatically in the GraphPad Prism program we used, and we did not find variance to be significantly different between the groups. 2 . Populations should be normally distributed or, more correctly, they should be sampled from a population that is normally distributed. Normality tests on the actual data have little value in terms of proving that the underlying population is normally distributed. However, it is often advocated to log-transform the data from gene expression data to better meet this assumption. We did not do this in this experiment, though it would probably have been prudent. However, ANOVA is generally fairly resistant to violations of this assumption (Motulsky, 2010). This assumption could also be circumvented by using a nonparametric test that does not assume normality; however, especially with low numbers of subjects in the groups, non-parametric tests have extremely low power. If there are only three subjects in each group, it is actually impossible to find a statistically significant difference with a nonparametric test.

By an almost incomprehensible oversight by us, the figures in the published paper did not contain markings of significance, even though the statistics are explained in the paper. Figures with the correct markings are included in this thesis and submitted to the journal as an erratum. 


\section{Paper II}

In this study, every rabbit served as its own control to account for the intrinsic variability in healing between animals. Our main outcome parameter was the filling, and to make sure we had sufficient statistical power, we performed a power analysis in the planning of the study with input from previous rabbit experiments in the group (Aroen, 2006). We considered a difference of $25 \%$ in filling between two treatments to be a "clinically" relevant threshold. We used a paired Student's t test to compare the degree of filling. This parametric test has the same assumption about normality as described above. With 11 subjects in each group, we could have chosen the non-parametric Wilcoxon match pairs test without losing too much power, even though it is not completely irrational to assume that the data were sampled from a normally distributed population. This test was used for the ordinal categorical variables from the semiquantitative evaluation of the repair.

We calculated the mean difference between measurements of observer one and two and between the first and second time a section was scored by the same observer to judge the inter- and intra-rater reliability. Only very minuscule differences existed, though we should most likely have calculated the intra-class coefficients as well to show this in a single value.

\section{Paper III}

We presented much of the data in this paper as individual donors, and the majority of the statistics is related to the global mRNA and microRNA profiling. When we performed statistical testing, it was along the lines of testing used in paper I, though we also used the Dennett's post hoc test, which is used when comparing the means of the groups to only one mean and not all combinations. We also performed log-transformations of the data to better meet the normality assumption. For the global profiling, we used a previously established line of analysis to both normalise and quality control the data (Karlsen, 2011). The subsequent testing for differential expression was performed using the rank product false discovery rate 
method (Breitling, 2004). This method is based on biological reasoning rather than just pure statistical thinking and calculates the rank products from replicate experiments. It also employs the false discovery rate as a more powerful and intuitive way of correcting for multiple testing than for example the highly conservative Bonferroni method. Explained in clear terms, the false discovery rate is the percentage of false positives expected among the discoveries (i.e., the rejected null-hypotheses). For example, if 100 genes are found to be differentially expressed with a FDR of 0.05 , it is expected that 5 of these 100 are false positives (but it remains unknown which are false).

\section{Paper IV}

The high-throughput methods used and the relatively high number of conditions tested in paper IV demanded a somewhat different statistical approach. First, to normalise the data from lysates, we needed more than one internal reference gene. We used the commonly used geometric mean method to more robustly achieve a good normalisation with five reference genes (Vandesompele, 2002). Visualisation of data is often a very powerful way to grasp the content of huge datasets, and several common principles to do this exist, such as heat maps and scatterplots. We utilised a standard principle known as Studentisation to centralise and normalise the data around a mean of 0 to be able to present graphically the variation related to the different treatments. To statistically test the graphical findings, we performed gene set enrichment tests by fitting a linear model to the data, as used previously for similar data (Ostrup, 2010). Here, we also employed FDR to account for the issue of multiple comparisons. Our customised chondrogenic gene set was composed of 364 genes.

Principal component analysis is a very powerful way of reducing the complexity of large datasets to elucidate the underlying variations (Li, 2010). This method calculates so-called components so that the first component explains the largest amount of variation in the data, the next component explains the largest amount of 
variation given that it is, statistically speaking, uncorrelated with the first component, and so forth. The analysis is usually restricted to two or three components judging by how much of the variation is explained by each of the components. One feasible way to analyse in connection with PCA is to look at which genes then contribute the most to each component. However, we instead focused on markers known to be relevant as either wanted for hyaline cartilage or related to alternative differentiation lineages or hypertrophic cartilage. 


\section{General discussion}

\section{Paper I}

Based on our findings presented here and in the literature discussed in the introduction, there are certainly indications that the use of human MSCs is one way to improve the cartilage repair procedures existing today. We found that type II collagen production could be induced in human BM-MSCs that by far exceeded the production observed in AT-MSCs and in unstimulated chondrocytes. In addition, in a recent paper from our group, BM-MSCs and chondrocytes from osteoarthritic cartilage were actually found to respond quite similarly to each other when subjected to differentiation factors in the same system as we used in papers III and IV (Fernandes, 2013). The difference in inducibility between MSCs from adipose tissue and bone marrow was found despite multiple global gene expression analyses showing MSC from various sources to be remarkably similar (Lee, 2004; Djouad, 2005; Wagner, 2005). However, the result is in line with other publications (Winter, 2003; Im, 2005; Afizah, 2007). At the time of the experiments, the reasons for this were not fully understood, but findings of reduced levels of BMP2, -4 and -6 and TGF $\beta$ receptor 1 (TGFBR1) and enhanced levels of integral membrane protein 2A (ITM2A) in AT-MSCs are most likely part of the explanation (Hennig, 2007; Boeuf, 2009). The reduced inducibility of ATMSCs has also been shown to be overcome by using BMP6 in addition to TGF $\beta 1$; however, in our case we already used BMP6 and still observed less induction. However, the true reason behind differences in inducibility from different sources of stem cells in general is likely due to epigenetic changes. The epigenetic control of cells is a multifaceted issue that includes not only the DNA methylation status, but also histone modifications, microRNAs and other regulatory molecules. MSCs from various sources have been studied for differences in DNA methylation status, but the comparison found only minor differences and not in gene regions likely to 
explain the differences in inducibility (Aranda, 2009; Sorensen, 2010). More likely, the differences are due to variability in histone modifications between the various stem cells. A detailed description of histone modification is beyond the scope of this thesis; briefly, histones around which the DNA is wrapped has tails of amino acids that can be modified, for instance by acetylation and methylation of specific amino acid residues. These modifications specify if the DNA region is primed, open, or closed to transcription. Histone modifications during chondrogenesis of BM-MSCs and of adipogenesis of AT-MSCs have been studied in detail (Mikkelsen, 2010; Herlofsen, 2013), but to the best of our knowledge, no one has directly compared histone modifications between undifferentiated ATand BM-MSCs. However, this comparison should be readily feasible from the raw data from the studies presented here.

There are some puzzling and potentially important findings in our study as well as some weaknesses. We found that although type II collagen was highly upregulated and produced when BM-MSCs received chondrogenic induction for the full duration of the culture period, it was not retained in the scaffold but rather secreted into the supernatant. The Hyaff-11-scaffold is a very loose mesh and has no inherent barrier to the diffusion of even very large molecules. This is a desired feature in regard to diffusion of nutrient and waste products; however, the retention of ECM is then completely dependent on the interlocking of molecules or connection to cell receptors or integrins. This property is a potential drawback to using this scaffold clinically, and results from the initial preclinical studies of this scaffold seemed to mirror the same pattern of cells clumped together with large open areas in the scaffold, although extracellular staining of collagens was observed (Grigolo, 2002; Facchini, 2006). Quite puzzlingly, we found the same intracellular distribution pattern of type I collagen, but we did not detect collagen I in the supernatant. We do not have any explanation for this, and, in retrospect, it should have been investigated more thoroughly. 
Another finding to highlight is the increase in type $\mathrm{X}$ collagen gene expression, which is not necessarily specific to this study but instead a general disadvantage of MSCs in chondrogenesis. Currently, it seems that in all cases in which MSCs are brought in the chondrogenic direction, type X collagen is induced (Boeuf, 2010; Herlofsen, 2011). Numerous studies have investigated ways to reduce this induction by adding other factors to the differentiation medium. Most convincingly, reduction, but not elimination, is observed with PTHRP, which opposes the Indian hedgehog pathway (Kim, 2008; Weiss, 2010). Perhaps type X collagen is primarily a problem of in vitro chondrogenesis, during which the differentiating cells do not get any spatial cues from the surrounding environment. For example, it has been shown that MSCs used to treat a cartilage defect in a minipig model did not express type $\mathrm{X}$ collagen other than in close proximity to the subchondral bone and in general showed better ratios between wanted and unwanted ECM molecules (Steck, 2009). Type I collagen is less often identified as a problem in in vitro chondrogenesis, but we also observed that it was highly expressed and upregulated. Most likely even more so than for type X collagen, persistent high gene and protein expression of type I collagen seems to be found in all differentiation systems. With the clinical findings of fibrocartilage after both $\mathrm{ACI}$ and spontaneous healing in human defects, this is an area for future research regardless of using chondrocytes or MSCs (Roberts, 2009).

\section{Paper II}

In the experimental study in rabbits, we did not reach a good filling of the defect, nor did we see a clear advantage of having MSCs in the scaffold. These results correlate well with the literature (Radice, 2000; Solchaga, 2005; Kayakabe, 2006), which shows that MSCs did not lead to improved filling of defects; however, the rate of degradability of the scaffolds was found to affect the repair. Also in these referenced studies, if cells had been cultured with fibroblast growth factor, then a positive effect was observed. 
We did see an increase in clustering, which could be argued to be a sign of a different repair response in the cell-loaded scaffold, indicating that adding cells does have an effect. In our model, we cannot conclude if this is a sign of cell division of the implanted cells or a trophic effect of the implanted cells on the cells invading from the bone marrow directly, or a combination of both. There are several publications that show that MSCs have trophic effects in tissue repair and that this may even be their most important function (Caplan, 2006). Conversely, there are also publications showing that implanted cells contribute directly to the repair of tissue (Oshima, 2005; Koga, 2008). The findings in paper I suggest that we might have observed a better repair in the rabbit studies had the MSCs been cultured for a longer time in the scaffold with growth factors. However, the inherent problem in our model and several other small animal models of cartilage repair is that the defects need to be osteochondral due to the very limited thickness of the articular cartilage in the rabbit. In the defects with cell-free scaffolds, we do see repair, presumably due to cells migrating from the bone marrow. Therefore, it is difficult to show the real advantages of having cells in the scaffolds.

In 2011, the ICRS published a white paper on preclinical studies in which this is discussed (Hurtig, 2011). They strongly advocate for the use of larger species, though they also acknowledge that if good histological confirmation of defect depth is used and if animals are mature, there is a lot of support for using the rabbit model. This was also the finding of a systematic review of animal models for cartilage repair (Ahern, 2009). Larger animal models that could be considered include minipigs, dogs, pigs, goats, sheep, or horses. Comparisons of the cartilage thickness of several species show that horse cartilage is closest to the thickness of human cartilage, approximately $2.5 \mathrm{~mm}$ (Frisbie, 2006). Few groups work primarily in a horse model, but it may yield results that can more easily be applied in the human setting (Koch, 2008; Frisbie, 2011; Fortier, 2012; Schnabel, 2013). Even keeping the disadvantages of the rabbit model or any small animal model in 
mind, it is clear that testing new treatments for cartilage repair in larger animals carries disadvantages of its own. The cost of both animals and animal care increases rapidly, and the dosing of antibiotics and analgesics is per $\mathrm{kg}$, which is considerably more in, for example, the horse model, also adding to the cost. In addition, rehabilitation issues become more important and difficult, though arguably these issues are often completely ignored in small animal models. Therefore, although it is not the optimal model, the rabbit model may still be the only practically available animal model for a large proportion of research groups. Perhaps there may be room for larger collaborative projects between research groups when going from smaller to larger animal models.

\section{Paper III}

The rationale for paper III came from previous studies in our lab of microRNA expression in native human chondrocytes undergoing dedifferentiation (Karlsen, 2011). We already knew that MSCs undergoing differentiation largely mirrored the gene expression of articular chondrocytes, although with some notable exceptions, such as type I and type X collagen expression. However, we did not know to what extent differentiated MSCs mirrored the microRNA profile of native chondrocytes. One would predict similar but not identical expression, and this was indeed what we found, further highlighting that the directed differentiation of MSCs into chondrocytes is not producing bona fide chondrocytes. However, we initially focused on the similarities, particularly the effect of miR-140, to complement the understanding of the role of this microRNA. As described in the introduction, miR-140 had recently been established to have an important role in cartilage homeostasis by targeting an aggrecanase ADAMTS5. However, strangely, it was not shown to target gene expression or translation of any of the major transcription factors or ECM-molecules. It was therefore an unexpected finding that the inhibition of miR-140-5p led to a clear downregulation of the aggrecan and SOX9 proteins, yet it did not have a clear effect on the 
corresponding mRNAs. The change in aggrecan might be at least partly explained by the known effect of miR-140 on ADAMTS5 (Miyaki, 2009), where less miR140 would lead to more ADAMTS5, which subsequently would lead to degradation of aggrecan protein. However, there was no obvious explanation of how miR-140 would directly lead to an upregulation of SOX9 protein levels.

Although microRNAs are commonly believed to exert most of their effect through canonical binding in the 3'UTR with ultimate downregulation of target gene protein expression, recent studies have discovered non-canonical pathways whereby microRNAs activate gene expression, sometimes through binding in the 5'UTR (Orom, 2008; Lin, 2011; Vasudevan, 2012; Meijer, 2013). We found potential binding sites for miR-140-5p in the 5'UTR of both SOX9 and aggrecan with similar predicted thermodynamic stabilities as the already validated binding sites. However, the literature is not abundant with examples of the activating effects of miRs, and we did not perform any experiments to validate whether miR140-5p bound the predicted sequences; therefore, it remains an open question if this mechanism contributes to the explanation in our experiments. Another way to explain the effect on SOX9 follows the same logic as for aggrecan and ADAMTS5: If miR-140 targets a gene that in turn inhibits protein expression of SOX9, this would potentially explain why SOX9 expression is decreased when miR-140 expression is repressed. This effect would also potentially add an additional feedback loop, as SOX9 is already known to control expression of miR140 expression in zebra fish (Nakamura, 2012). We identified several potential targets of miR-140-5p and $-3 p$ and validated $R A L A$ as target. Surprisingly but very interestingly, we found that knocking down RALA led to an increase in SOX9 protein levels without affecting SOX9 mRNA or actin protein. This suggests that the effect of inhibiting miR-140 on SOX9 could be an effect of RALA on the translation of SOX9. However, exactly how this occurs and how this affects in vitro chondrogenesis is another unanswered question. 
RALA has not previously been described to be important for chondrogenesis. RALA is a small GTPase involved in TGF $\beta$ /activin signalling (Kardassis, 2009). It contributes to the internalisation of activin receptors, thus reducing the number of receptors available for ligand interaction, which in turn could inhibit signalling. The activin A receptor was shown to be positively linked to chondrogenesis, with type II collagen and sulphated GAGs being reduced when the activin A receptor was knocked down (Djouad, 2010). Furthermore, RALA has been shown to inhibit exocytosis (Wang, 2004). Aggrecan and its associated GAGs are transported through the endoplasmic reticulum-Golgi pathway before being secreted from the cell surface (Luo, 2000). miR-181a has interestingly also been shown to target RALA (Fei, 2012) and very recently was shown to interfere with aggrecan expression through an indirect mechanism (Sumiyoshi, 2013). We also found miR-181a to be upregulated during chondrogenesis of MSCs; however, it was only barely detected in uncultured chondrocytes.

We gathered large datasets for paper III and were not able to follow up on all interesting microRNAs. For example, miR-21 has been shown to be upregulated by TGF $\beta$ and has been associated with increased type I collagen synthesis in some experiments (Kim, 2010a). A change from type II to type I collagen synthesis is a hallmark of dedifferentiating chondrocytes (Holtzer, 1960). Consistent with this, miR-21 was also one of the upregulated microRNAs during dedifferentiation and also when MSCs differentiate, possibly explaining why type I collagen synthesis is kept high during the directed differentiation of MSCs. miR-210 is commonly labelled as a hypoxic microRNA because it is upregulated under hypoxic conditions (Chan, 2012). We found that miR-210 was gradually upregulated during chondrogenesis and downregulated during dedifferentiation. The upregulation would be consistent with increasingly hypoxic conditions in the three-dimensional cultures, where matrix production and increased metabolism may lead to decreased oxygen diffusion into discs. We observed that miR-335 is 
downregulated during chondrogenesis. This is in line with already published findings that the downregulation of miR-335 is critical for MSC differentiation (Tome, 2011; Zhang, 2011) but in contrast to a recent study in mouse showing that miR-335 promotes chondrogenesis (Lin, 2013). Recently, miR-574-3p was shown to inhibit MSC chondrogenesis (Guerit, 2013). Interestingly, we found this microRNA to be significantly upregulated at day 21 in differentiating MSCs and the complementary miR-574-5p to be upregulated at day 7 in dedifferentiating chondrocytes.

In general, our findings in paper III illustrate that microRNAs play important roles in in vitro chondrogenesis and could be manipulated to improve the current protocols of in vitro chondrogenesis.

\section{Paper IV}

In paper IV, we used factorial analysis and high-throughput gene expression profiling to dissect the expression of a chondrogenesis-relevant gene set during in vitro chondrogenesis of MSCs subjected to 48 different conditions of growth factors and cell densities. We aimed to validate this approach for studying directed differentiation and to find out if presently used factors could be combined more cleverly to promote a better differentiation cocktail. The main findings were stated above, and the discussion in the paper shows that our findings are largely in line with the published literature. Perhaps most interestingly, we found that no matter which combination of factors was used, upregulating wanted chondrogenic markers also upregulated unwanted markers. The only exception was that adding dexamethasone to TGF $\beta$ or BMP2 generally led to favourable changes, such as a downregulation of osteocalcin $(B G L A P)$, though not to an elimination of unwanted markers. These results further support the findings from paper I and III that the current differentiation cocktails do not lead to a perfect hyaline differentiation. 
We propose that the method used in paper IV may provide a suitable way to improve the current protocols; however, it is important to remember the limitation that we only considered gene expression at the level of transcribed mRNA, which does not necessarily correlate with protein synthesis (Schwanhausser, 2011). We found that profiling could be performed directly on lysates and that changes in gene expression observed after just one day of induction predicted later changes. We also found that the findings from our analysis were largely supported by the literature. Taken together, we believe this way of studying directed differentiation is sound and could be expanded to larger experiments. For example, it would be interesting to plan further experiments with a more elaborate design in terms of concentration of factors. This would allow for statistical analysis with response optimisation to be performed instead of the more crude two-level design of either absence or presence (Enochson, 2012). 


\section{Conclusions and future studies}

More often than not, research, and perhaps basic science research in particular, raises more questions than it answers. This remains true for the presented studies. Here, a brief list is given of the general conclusions of our studies and the future studies that could be pursued.

\section{Bone marrow MSCs were more easily induced into the chondrogenic lineage than adipose tissue MSCs.}

Even though the experiments leading to this conclusion were performed several years ago, there is still no simple explanation as to why MSCs from various tissues, although remarkably similar, have different differentiation capacities. One obvious way to study this further would be to compare the different cell sources epigenetically, looking at histone modifications, which could be conducted using publicly available data (Mikkelsen, 2010; Herlofsen, 2013) and possibly also looking at global microRNA profiles. This might help to determine if one cell source should be preferred for cartilage tissue engineering.

\section{MSCs in a hyaluronic acid scaffold did not lead to better filling in a rabbit model, though there was a tendency of better quality repair tissue in the cell- treated defects.}

Tissue-engineered constructs need functional verification in animal models. The rabbit model seems to be the most practical first model in which to test new tissue engineered constructs; however, larger animals could provide a test situation more similar to human. There are also studies showing that xenotransplantation is possible with human cells in larger animals, which would also advocate a move to larger species for future animal experiments (Li, 2012). 


\section{The microRNA profile of chondrogenically differentiated MSCs resembled that of uncultured chondrocytes, though they were not identical. Several microRNAs showed a reciprocal relationship during the dedifferentiation of chondrocytes and chondrogenic differentiation of MSCs.}

Several future experiments could be pursued based on these findings. Currently, we are looking at the role of miR-221 in chondrogenesis, which was one of the microRNAs identified to be reciprocally regulated. It would also be interesting to look further into the differences in the microRNA profile between native chondrocytes and differentiated MSCs to see if there could be clues to the unwanted production of ECM, such as type I and X collagen.

In addition, in vivo studies of the therapeutic potential of microRNAs in disease models related to bone or cartilage pathology could be a path to follow.

Finally, testing if extracellular microRNAs in synovial fluid, plasma or even urine could be used as diagnostic or prognostic markers in skeletal diseases would be an interesting study to pursue.

4. miR-140 had a profound impact on in vitro chondrogenesis with a positive effect on SOX9 and aggrecan post-transcriptionally, possibly, at least partly, through RALA, which we identified as being a target of miR-140-5p.

Further studies of RALA and activin signalling in the context of chondrogenesis are warranted. How does RALA influence the translation of SOX9 and does this influence extend to other genes? Is this signalling pathway a major player in or just a fine-tuner of chondrogenesis? 
Additionally, exploring the hypothesis of 5'UTR binding of miR-140-5p in SOX9 and $A C A N$ would be natural, possibly with a combination of luciferase reporter assays with the 5'UTR sequences and co-immunoprecipitation of miR-140-5p.

\section{Better differentiation cocktails are needed to avoid the expression of} unwanted markers, such as type $I$ and $X$ collagen. This cannot be achieved by the commonly used differentiation factors, but screening for new molecules might be performed in a multifactorial fashion using quality by design and high-throughput profiling with a limited set of chondrogenesis-related genes.

High-throughput screening in tissue engineering has led to important discoveries, highlighted for example by the discovery of kartogenin as small chondrogenic molecule (Johnson, 2012). The current protocols for differentiation are not perfect. Screens for modifiers of in vitro chondrogenesis with particular focus on eliminating the expression of unwanted ECM production would be an interesting path to explore, as would a more temporal adaptation of in vitro chondrogenesis to embryogenesis of cartilage in vitro (Wu, 2013). 


\section{References}

Abbott, A. (2013). Doubt cast over tiny stem cells. Nature 499, 390.

Afizah, H., Yang, Z., Hui, J.H., Ouyang, H.W., and Lee, E.H. (2007). A comparison between the chondrogenic potential of human bone marrow stem cells (BMSCs) and adipose-derived stem cells (ADSCs) taken from the same donors. Tissue Eng. 13, 659-666.

Aguiar, D.J., Knudson, W., and Knudson, C.B. (1999). Internalization of the hyaluronan receptor CD44 by chondrocytes. Exp. Cell Res. 252, 292-302.

Ahern, B.J., Parvizi, J., Boston, R., and Schaer, T.P. (2009). Preclinical animal models in single site cartilage defect testing: a systematic review. Osteoarthritis Cartilage 17, 705-713.

Akiyama, H. (2008). Control of chondrogenesis by the transcription factor Sox9. Modern rheumatology / the Japan Rheumatism Association 18, 213-219.

Akiyama, H., Chaboissier, M.C., Martin, J.F., Schedl, A., and de Crombrugghe, B. (2002). The transcription factor Sox9 has essential roles in successive steps of the chondrocyte differentiation pathway and is required for expression of Sox 5 and Sox6. Genes Dev. 16, 2813-2828.

Alberts, B. (2002). Molecular biology of the cell. Garland Science: New York.

Alfredson, H., and Lorentzon, R. (1999). Superior results with continuous passive motion compared to active motion after periosteal transplantation. A retrospective study of human patella cartilage defect treatment. Knee Surg. Sports Traumatol. Arthrosc. 7, 232-238.

Alsalameh, S., Amin, R., Gemba, T., and Lotz, M. (2004). Identification of mesenchymal progenitor cells in normal and osteoarthritic human articular cartilage. Arthritis Rheum. 50, 1522-1532.

Alvarez-Dolado, M. (2007). Cell fusion: biological perspectives and potential for regenerative medicine. Front. Biosci. 12, 1-12.

Ambros, V. (2004). The functions of animal microRNAs. Nature 431, 350-355.

Andersen, T., Strand, B.L., Formo, K., Alsberg, B.E., and Christensen, B.E. (2012). Alginates as biomaterials in tissue engineering. Carbohydrate Chemistry 37, 227-258.

Anokye-Danso, F., Trivedi, C.M., Juhr, D., Gupta, M., Cui, Z., Tian, Y., Zhang, Y., Yang, W., Gruber, P.J., Epstein, J.A., and Morrisey, E.E. (2011). Highly efficient miRNA-mediated reprogramming of mouse and human somatic cells to pluripotency. Cell Stem Cell 8, 376-388.

Araldi, E., and Schipani, E. (2010). MicroRNA-140 and the silencing of osteoarthritis. Genes Dev. 24, $1075-1080$

Aranda, P., Agirre, X., Ballestar, E., Andreu, E.J., Roman-Gomez, J., Prieto, I., Martin-Subero, J.I., Cigudosa, J.C., Siebert, R., Esteller, M., and Prosper, F. (2009). Epigenetic signatures associated with different levels of differentiation potential in human stem cells. PloS one 4, e7809.

Aroen, A., Heir, S., Loken, S., Engebretsen, L., and Reinholt, F.P. (2006). Healing of articular cartilage defects. An experimental study of vascular and minimal vascular microenvironment. J. Orthop. Res. 24, 1069-1077. 
Aroen, A., Loken, S., Heir, S., Alvik, E., Ekeland, A., Granlund, O.G., and Engebretsen, L. (2004). Articular cartilage lesions in 993 consecutive knee arthroscopies. Am. J. Sports Med. 32, 211-215.

Aurich, M., Poole, A.R., Reiner, A., Mollenhauer, C., Margulis, A., Kuettner, K.E., and Cole, A.A. (2002). Matrix homeostasis in aging normal human ankle cartilage. Arthritis Rheum. 46, 2903-2910.

Axe, J.M., Snyder-Mackler, L., and Axe, M.J. (2013). The role of viscosupplementation. Sports Med Arthrosc 21, 18-22.

Ball, L.M., Bernardo, M.E., Roelofs, H., van Tol, M.J., Contoli, B., Zwaginga, J.J., Avanzini, M.A., Conforti, A., Bertaina, A., Giorgiani, G., Jol-van der Zijde, C.M., Zecca, M., Le Blanc, K., Frassoni, F., Egeler, R.M., Fibbe, W.E., Lankester, A.C., and Locatelli, F. (2013). Multiple infusions of mesenchymal stromal cells induce sustained remission in children with steroid-refractory, grade III-IV acute graft-versushost disease. Br. J. Haematol. 163, 501-509.

Baron, F., Storb, R., and Little, M.T. (2003). Hematopoietic cell transplantation: five decades of progress. Arch. Med. Res. 34, 528-544.

Bartel, D.P. (2004). MicroRNAs: genomics, biogenesis, mechanism, and function. Cell 116, 281-297.

Bartel, D.P. (2009). MicroRNAs: target recognition and regulatory functions. Cell 136, 215-233.

Behery, O., Siston, R.A., Harris, J.D., and Flanigan, D.C. (2013). Treatment of Cartilage Defects of the Knee: Expanding on the Existing Algorithm. Clin. J. Sport Med.

Benjamin, M., Archer, C.W., and Ralphs, J.R. (1994). Cytoskeleton of cartilage cells. Microsc. Res. Tech. 28, 372-377.

Bentley, G., Biant, L.C., Carrington, R.W., Akmal, M., Goldberg, A., Williams, A.M., Skinner, J.A., and Pringle, J. (2003). A prospective, randomised comparison of autologous chondrocyte implantation versus mosaicplasty for osteochondral defects in the knee. J. Bone Joint Surg. Br. 85, 223-230.

Bernard, P. (2003). Dimerization of SOX9 is required for chondrogenesis, but not for sex determination. Hum. Mol. Genet. 12, 1755-1765.

Bi, W., Deng, J.M., Zhang, Z., Behringer, R.R., and de Crombrugghe, B. (1999). Sox9 is required for cartilage formation. Nat. Genet. 22, 85-89.

Bianco, P., Riminucci, M., Gronthos, S., and Robey, P.G. (2001). Bone marrow stromal stem cells: nature, biology, and potential applications. Stem Cells 19, 180-192.

Bland, Y.S., and Ashhurst, D.E. (1996). Development and ageing of the articular cartilage of the rabbit knee joint: distribution of the fibrillar collagens. Anat. Embryol. (Berl). 194, 607-619.

Blevins, F.T., Steadman, J.R., Rodrigo, J.J., and Silliman, J. (1998). Treatment of articular cartilage defects in athletes: an analysis of functional outcome and lesion appearance. Orthopedics 21, 761-767; discussion 767-768.

Boeuf, S., Borger, M., Hennig, T., Winter, A., Kasten, P., and Richter, W. (2009). Enhanced ITM2A expression inhibits chondrogenic differentiation of mesenchymal stem cells. Differentiation 78, 108-115.

Boeuf, S., and Richter, W. (2010). Chondrogenesis of mesenchymal stem cells: role of tissue source and inducing factors. Stem cell research \& therapy 1,31 . 
Bonaventure, J., Kadhom, N., Cohen-Solal, L., Ng, K.H., Bourguignon, J., Lasselin, C., and Freisinger, P. (1994). Reexpression of cartilage-specific genes by dedifferentiated human articular chondrocytes cultured in alginate beads. Exp. Cell Res. 212, 97-104.

Boquest, A.C., Shahdadfar, A., Brinchmann, J.E., and Collas, P. (2006). Isolation of stromal stem cells from human adipose tissue. Methods Mol. Biol. 325, 35-46.

Boquest, A.C., Shahdadfar, A., Fronsdal, K., Sigurjonsson, O., Tunheim, S.H., Collas, P., and Brinchmann, J.E. (2005). Isolation and transcription profiling of purified uncultured human stromal stem cells: alteration of gene expression after in vitro cell culture. Mol. Biol. Cell 16, 1131-1141.

Breitling, R., Armengaud, P., Amtmann, A., and Herzyk, P. (2004). Rank products: a simple, yet powerful, new method to detect differentially regulated genes in replicated microarray experiments. FEBS Lett. 573, 83-92.

Brennecke, J., Stark, A., Russell, R.B., and Cohen, S.M. (2005). Principles of microRNA-target recognition. PLoS biology 3, e85.

Brittberg, M., Lindahl, A., Nilsson, A., Ohlsson, C., Isaksson, O., and Peterson, L. (1994). Treatment of deep cartilage defects in the knee with autologous chondrocyte transplantation. N. Engl. J. Med. 331, 889895 .

Brittberg, M., and Peterson, L. (1998). Introduction of an articular cartilage classification. ICRS Newsletter $1,5-8$.

Brittberg, M., and Winalski, C.S. (2003). Evaluation of cartilage injuries and repair. J. Bone Joint Surg. Am. 85-A Suppl 2, 5-8.

Brzoska, M., Geiger, H., Gauer, S., and Baer, P. (2005). Epithelial differentiation of human adipose tissuederived adult stem cells. Biochem. Biophys. Res. Commun. 330, 142-150.

Cao, B., and Huard, J. (2004). Muscle-derived stem cells. Cell cycle (Georgetown, Tex 3, 104-107.

Cao, Y., Sun, Z., Liao, L., Meng, Y., Han, Q., and Zhao, R.C. (2005). Human adipose tissue-derived stem cells differentiate into endothelial cells in vitro and improve postnatal neovascularization in vivo. Biochem. Biophys. Res. Commun. 332, 370-379.

Caplan, A.I. (1991). Mesenchymal stem cells. J. Orthop. Res. 9, 641-650.

Caplan, A.I. (2005). Review: mesenchymal stem cells: cell-based reconstructive therapy in orthopedics. Tissue Eng. 11, 1198-1211.

Caplan, A.I. (2010). What's in a Name? Tissue Eng Part A.

Caplan, A.I., and Dennis, J.E. (2006). Mesenchymal stem cells as trophic mediators. J. Cell. Biochem. 98 , 1076-1084.

Castillo, E.C., and Kouri, J.B. (2004). A new role for chondrocytes as non-professional phagocytes. An in vitro study. Microsc. Res. Tech. 64, 269-268.

Centers for Disease Control and Prevention. (2002). Update: allograft-associated bacterial infections-United States, 2002. MMWR. Morb. Mortal. Wkly. Rep. 51, 207-210.

(2010). Prevalence of doctor-diagnosed arthritis and arthritis-attributable activity limitation --- United States, 2007-2009. Centers for disease control and prevention, 1545-861X (Electronic) 0149-2195 (Linking). 
Chamberlain, G., Fox, J., Ashton, B., and Middleton, J. (2007). Mesenchymal Stem Cells: their Phenotype, Differentiation Capacity, Immunological Features and Potential for Homing. Stem Cells.

Chan, Y.C., Banerjee, J., Choi, S.Y., and Sen, C.K. (2012). miR-210: The Master Hypoxamir. Microcirculation 19, 215-223.

Chen, H., Chevrier, A., Hoemann, C.D., Sun, J., Ouyang, W., and Buschmann, M.D. (2011a).

Characterization of subchondral bone repair for marrow-stimulated chondral defects and its relationship to articular cartilage resurfacing. Am. J. Sports Med. 39, 1731-1740.

Chen, H., Sun, J., Hoemann, C.D., Lascau-Coman, V., Ouyang, W., McKee, M.D., Shive, M.S., and Buschmann, M.D. (2009). Drilling and microfracture lead to different bone structure and necrosis during bone-marrow stimulation for cartilage repair. J. Orthop. Res. 27, 1432-1438.

Chen, J., Wang, C., Lu, S., Wu, J., Guo, X., Duan, C., Dong, L., Song, Y., Zhang, J., Jing, D., Wu, L., Ding, J., and Li, D. (2005). In vivo chondrogenesis of adult bone-marrow-derived autologous mesenchymal stem cells. Cell Tissue Res. 319, 429-438.

Chen, Y., Bloemen, V., Impens, S., Moesen, M., Luyten, F.P., and Schrooten, J. (2011b). Characterization and optimization of cell seeding in scaffolds by factorial design: quality by design approach for skeletal tissue engineering. Tissue engineering. Part C, Methods 17, 1211-1221.

Christensen, S.E., Coles, J.M., Zelenski, N.A., Furman, B.D., Leddy, H.A., Zauscher, S., Bonaldo, P., and Guilak, F. (2012). Altered trabecular bone structure and delayed cartilage degeneration in the knees of collagen VI null mice. PloS one 7, e33397.

Colnot, C. (2005). Cellular and molecular interactions regulating skeletogenesis. J. Cell. Biochem. 95, 688697.

Crapnell, K., Blaesius, R., Hastings, A., Lennon, D.P., Caplan, A.I., and Bruder, S.P. (2013). Growth, differentiation capacity, and function of mesenchymal stem cells expanded in serum-free medium developed via combinatorial screening. Exp. Cell Res. 319, 1409-1418.

Crelin, E.S. (1957). Mitosis in adult cartilage. Science 125, 650.

Cristino, S., Grassi, F., Toneguzzi, S., Piacentini, A., Grigolo, B., Santi, S., Riccio, M., Tognana, E., Facchini, A., and Lisignoli, G. (2005). Analysis of mesenchymal stem cells grown on a three-dimensional HYAFF 11-based prototype ligament scaffold. Journal of biomedical materials research. Part A 73, 275283.

Curl, W.W., Krome, J., Gordon, E.S., Rushing, J., Smith, B.P., and Poehling, G.G. (1997). Cartilage injuries: a review of 31,516 knee arthroscopies. Arthroscopy 13, 456-460.

D'Ippolito, G., Diabira, S., Howard, G.A., Menei, P., Roos, B.A., and Schiller, P.C. (2004). Marrowisolated adult multilineage inducible (MIAMI) cells, a unique population of postnatal young and old human cells with extensive expansion and differentiation potential. J. Cell Sci. 117, 2971-2981.

D'Lima, D.D., Hashimoto, S., Chen, P.C., Colwell, C.W., Jr., and Lotz, M.K. (2001a). Human chondrocyte apoptosis in response to mechanical injury. Osteoarthritis Cartilage 9, 712-719.

D'Lima, D.D., Hashimoto, S., Chen, P.C., Colwell, C.W., Jr., and Lotz, M.K. (2001b). Impact of mechanical trauma on matrix and cells. Clin Orthop Relat Res, S90-99.

da Silva Meirelles, L., Chagastelles, P.C., and Nardi, N.B. (2006). Mesenchymal stem cells reside in virtually all post-natal organs and tissues. J. Cell Sci. 119, 2204-2213. 
De Bari, C., Dell'Accio, F., Tylzanowski, P., and Luyten, F.P. (2001). Multipotent mesenchymal stem cells from adult human synovial membrane. Arthritis Rheum. 44, 1928-1942.

De Bari, C., Dell'Accio, F., Vanlauwe, J., Eyckmans, J., Khan, I.M., Archer, C.W., Jones, E.A., McGonagle, D., Mitsiadis, T.A., Pitzalis, C., and Luyten, F.P. (2006). Mesenchymal multipotency of adult human periosteal cells demonstrated by single-cell lineage analysis. Arthritis Rheum. 54, 1209-1221.

de Crombrugghe, B., Lefebvre, V., and Nakashima, K. (2001). Regulatory mechanisms in the pathways of cartilage and bone formation. Curr. Opin. Cell Biol. 13, 721-727.

De Guire, V., Robitaille, R., Tetreault, N., Guerin, R., Menard, C., Bambace, N., and Sapieha, P. (2013). Circulating miRNAs as sensitive and specific biomarkers for the diagnosis and monitoring of human diseases: promises and challenges. Clin. Biochem. 46, 846-860.

Derynck, R., and Akhurst, R.J. (2007). Differentiation plasticity regulated by TGF-beta family proteins in development and disease. Nature cell biology 9, 1000-1004.

Derynck, R., Gelbart, W.M., Harland, R.M., Heldin, C.H., Kern, S.E., Massague, J., Melton, D.A., Mlodzik, M., Padgett, R.W., Roberts, A.B., Smith, J., Thomsen, G.H., Vogelstein, B., and Wang, X.F. (1996). Nomenclature: vertebrate mediators of TGFbeta family signals. Cell $87,173$.

Diaz-Romero, J., Gaillard, J.P., Grogan, S.P., Nesic, D., Trub, T., and Mainil-Varlet, P. (2005). Immunophenotypic analysis of human articular chondrocytes: changes in surface markers associated with cell expansion in monolayer culture. J. Cell. Physiol. 202, 731-742.

Diaz-Romero, J., Nesic, D., Grogan, S.P., Heini, P., and Mainil-Varlet, P. (2007). Immunophenotypic changes of human articular chondrocytes during monolayer culture reflect bona fide dedifferentiation rather than amplification of progenitor cells. J. Cell. Physiol. 214, 75-83.

Dickhut, A., Pelttari, K., Janicki, P., Wagner, W., Eckstein, V., Egermann, M., and Richter, W. (2009). Calcification or dedifferentiation: requirement to lock mesenchymal stem cells in a desired differentiation stage. J. Cell. Physiol. 219, 219-226.

Diez Villanueva, P., Sanz-Ruiz, R., Nunez Garcia, A., Fernandez Santos, M.E., Sanchez, P.L., and Fernandez-Aviles, F. (2012). Functional multipotency of stem cells: what do we need from them in the heart? Stem cells international 2012,817364.

Djouad, F., Bony, C., Haupl, T., Uze, G., Lahlou, N., Louis-Plence, P., Apparailly, F., Canovas, F., Reme, T., Sany, J., Jorgensen, C., and Noel, D. (2005). Transcriptional profiles discriminate bone marrow-derived and synovium-derived mesenchymal stem cells. Arthritis Res Ther 7, R1304-1315.

Djouad, F., Jackson, W.M., Bobick, B.E., Janjanin, S., Song, Y., Huang, G.T., and Tuan, R.S. (2010). Activin A expression regulates multipotency of mesenchymal progenitor cells. Stem cell research \& therapy 1,11 .

Doherty, M.J., Ashton, B.A., Walsh, S., Beresford, J.N., Grant, M.E., and Canfield, A.E. (1998). Vascular pericytes express osteogenic potential in vitro and in vivo. J. Bone Miner. Res. 13, 828-838.

Dominici, M., Le Blanc, K., Mueller, I., Slaper-Cortenbach, I., Marini, F., Krause, D., Deans, R., Keating, A., Prockop, D., and Horwitz, E. (2006). Minimal criteria for defining multipotent mesenchymal stromal cells. The International Society for Cellular Therapy position statement. Cytotherapy 8, 315-317.

Dowthwaite, G.P., Bishop, J.C., Redman, S.N., Khan, I.M., Rooney, P., Evans, D.J., Haughton, L., Bayram, Z., Boyer, S., Thomson, B., Wolfe, M.S., and Archer, C.W. (2004). The surface of articular cartilage contains a progenitor cell population. J. Cell Sci. 117, 889-897. 
Dozin, B., Malpeli, M., Cancedda, R., Bruzzi, P., Calcagno, S., Molfetta, L., Priano, F., Kon, E., and Marcacci, M. (2005). Comparative evaluation of autologous chondrocyte implantation and mosaicplasty: a multicentered randomized clinical trial. Clin. J. Sport Med. 15, 220-226.

Dudek, K.A., Lafont, J.E., Martinez-Sanchez, A., and Murphy, C.L. (2010). Type II collagen expression is regulated by tissue-specific miR-675 in human articular chondrocytes. J. Biol. Chem. 285, 24381-24387.

Duggal, S., Fronsdal, K.B., Szoke, K., Shahdadfar, A., Melvik, J.E., and Brinchmann, J.E. (2009). Phenotype and gene expression of human mesenchymal stem cells in alginate scaffolds. Tissue Eng Part A $15,1763-1773$.

Dunn, W., DuRaine, G., and Reddi, A.H. (2009). Profiling microRNA expression in bovine articular cartilage and implications for mechanotransduction. Arthritis Rheum. 60, 2333-2339.

Durand, S., and Cimarelli, A. (2011). The inside out of lentiviral vectors. Viruses 3, 132-159.

Dy, P., Wang, W., Bhattaram, P., Wang, Q., Wang, L., Ballock, R.T., and Lefebvre, V. (2012). Sox9 directs hypertrophic maturation and blocks osteoblast differentiation of growth plate chondrocytes. Developmental cell 22, 597-609.

Enea, D., Cecconi, S., Busilacchi, A., Manzotti, S., Gesuita, R., and Gigante, A. (2012). Matrix-induced autologous chondrocyte implantation (MACI) in the knee. Knee Surg. Sports Traumatol. Arthrosc. 20, $862-$ 869 .

English, K. (2013). Mechanisms of mesenchymal stromal cell immunomodulation. Immunol. Cell Biol. 91, 19-26.

Enochson, L., Brittberg, M., and Lindahl, A. (2012). Optimization of a chondrogenic medium through the use of factorial design of experiments. BioResearch open access 1, 306-313.

Esquela-Kerscher, A., Trang, P., Wiggins, J.F., Patrawala, L., Cheng, A., Ford, L., Weidhaas, J.B., Brown, D., Bader, A.G., and Slack, F.J. (2008). The let-7 microRNA reduces tumor growth in mouse models of lung cancer. Cell cycle (Georgetown, Tex 7, 759-764.

Eyre, D.R. (2004). Collagens and cartilage matrix homeostasis. Clin Orthop Relat Res, S118-122.

Facchini, A., Lisignoli, G., Cristino, S., Roseti, L., De Franceschi, L., Marconi, E., and Grigolo, B. (2006). Human chondrocytes and mesenchymal stem cells grown onto engineered scaffold. Biorheology 43, 471480 .

Falkenberg, N., Anastasov, N., Rappl, K., Braselmann, H., Auer, G., Walch, A., Huber, M., Hofig, I., Schmitt, M., Hofler, H., Atkinson, M.J., and Aubele, M. (2013). MiR-221/-222 differentiate prognostic groups in advanced breast cancers and influence cell invasion. Br. J. Cancer 109, 2714-2723.

Fei, J., Li, Y., Zhu, X., and Luo, X. (2012). miR-181a post-transcriptionally downregulates oncogenic RalA and contributes to growth inhibition and apoptosis in chronic myelogenous leukemia (CML). PloS one 7 , e32834.

Feng, J., Mantesso, A., De Bari, C., Nishiyama, A., and Sharpe, P.T. (2011). Dual origin of mesenchymal stem cells contributing to organ growth and repair. Proc. Natl. Acad. Sci. U. S. A. 108, 6503-6508.

Feng, X.H., and Derynck, R. (2005). Specificity and versatility in tgf-beta signaling through Smads. Annu. Rev. Cell Dev. Biol. 21, 659-693. 
Fernandes, A.M., Herlofsen, S.R., Karlsen, T.A., Kuchler, A.M., Floisand, Y., and Brinchmann, J.E. (2013). Similar properties of chondrocytes from osteoarthritis joints and mesenchymal stem cells from healthy donors for tissue engineering of articular cartilage. PloS one 8, e62994.

Findikli, N., Candan, N.Z., and Kahraman, S. (2006). Human embryonic stem cell culture: current limitations and novel strategies. Reproductive biomedicine online 13, 581-590.

Flanigan, D.C., Harris, J.D., Trinh, T.Q., Siston, R.A., and Brophy, R.H. (2010). Prevalence of chondral defects in athletes' knees: a systematic review. Med. Sci. Sports Exerc. 42, 1795-1801.

Flannery, C.R., Hughes, C.E., Schumacher, B.L., Tudor, D., Aydelotte, M.B., Kuettner, K.E., and Caterson, B. (1999). Articular cartilage superficial zone protein (SZP) is homologous to megakaryocyte stimulating factor precursor and Is a multifunctional proteoglycan with potential growth-promoting, cytoprotective, and lubricating properties in cartilage metabolism. Biochem. Biophys. Res. Commun. 254, 535-541.

Fortier, L.A. (2012). Making progress in the what, when and where of regenerative medicine for our equine patients. Equine Vet. J. 44, 511-512.

Fortina, P., and Surrey, S. (2008). Digital mRNA profiling. Nat. Biotechnol. 26, 293-294.

Foster, J.W., Dominguez-Steglich, M.A., Guioli, S., Kwok, C., Weller, P.A., Stevanovic, M., Weissenbach, J., Mansour, S., Young, I.D., Goodfellow, P.N., and et al. (1994). Campomelic dysplasia and autosomal sex reversal caused by mutations in an SRY-related gene. Nature 372, 525-530.

Francois, S., Bensidhoum, M., Mouiseddine, M., Mazurier, C., Allenet, B., Semont, A., Frick, J., Sache, A., Bouchet, S., Thierry, D., Gourmelon, P., Gorin, N.C., and Chapel, A. (2006). Local irradiation not only induces homing of human mesenchymal stem cells at exposed sites but promotes their widespread engraftment to multiple organs: a study of their quantitative distribution after irradiation damage. Stem Cells 24, 1020-1029.

Frantz, S. (2012). Embryonic stem cell pioneer Geron exits field, cuts losses. Nat. Biotechnol. 30, 12-13.

Frenette, P.S., Pinho, S., Lucas, D., and Scheiermann, C. (2013). Mesenchymal stem cell: keystone of the hematopoietic stem cell niche and a stepping-stone for regenerative medicine. Annu. Rev. Immunol. 31, 285-316.

Friedenstein, A.J., Chailakhjan, R.K., and Lalykina, K.S. (1970). The development of fibroblast colonies in monolayer cultures of guinea-pig bone marrow and spleen cells. Cell Tissue Kinet. 3, 393-403.

Friedman, R.C., Farh, K.K., Burge, C.B., and Bartel, D.P. (2009). Most mammalian mRNAs are conserved targets of microRNAs. Genome Res. 19, 92-105.

Frisbie, D.D., Cross, M.W., and McIlwraith, C.W. (2006). A comparative study of articular cartilage thickness in the stifle of animal species used in human pre-clinical studies compared to articular cartilage thickness in the human knee. Vet Comp Orthop Traumatol 19, 142-146.

Frisbie, D.D., and Stewart, M.C. (2011). Cell-based therapies for equine joint disease. Vet. Clin. North Am. Equine Pract. 27, 335-349.

Ge, Z., Li, C., Heng, B.C., Cao, G., and Yang, Z. (2012). Functional biomaterials for cartilage regeneration. Journal of biomedical materials research. Part A 100, 2526-2536.

Geiss, G.K., Bumgarner, R.E., Birditt, B., Dahl, T., Dowidar, N., Dunaway, D.L., Fell, H.P., Ferree, S., George, R.D., Grogan, T., James, J.J., Maysuria, M., Mitton, J.D., Oliveri, P., Osborn, J.L., Peng, T., Ratcliffe, A.L., Webster, P.J., Davidson, E.H., Hood, L., and Dimitrov, K. (2008). Direct multiplexed measurement of gene expression with color-coded probe pairs. Nat. Biotechnol. 26, 317-325. 
Geneser, F., and Dørup, J. (1999). Histology: Molecular biology based text book. [Histologi: på molekylærbiologisk grundlag.]. Munksgaard: København.

Gharravi, A.M., Orazizadeh, M., Ansari-Asl, K., Banoni, S., Izadi, S., and Hashemitabar, M. (2012). Design and fabrication of anatomical bioreactor systems containing alginate scaffolds for cartilage tissue engineering. Avicenna J Med Biotechnol 4, 65-74.

Goldring, M.B. (2006a). Are bone morphogenetic proteins effective inducers of cartilage repair? Ex vivo transduction of muscle-derived stem cells. Arthritis Rheum. 54, 387-389.

Goldring, M.B. (2012). Chondrogenesis, chondrocyte differentiation, and articular cartilage metabolism in health and osteoarthritis. Ther Adv Musculoskelet Dis 4, 269-285.

Goldring, M.B., Tsuchimochi, K., and Ijiri, K. (2006b). The control of chondrogenesis. J. Cell. Biochem. $97,33-44$.

Granan, L.P., Forssblad, M., Lind, M., and Engebretsen, L. (2009). The Scandinavian ACL registries 20042007: baseline epidemiology. Acta orthopaedica 80, 563-567.

Green, W.T., Jr. (1971). Behavior of articular chondrocytes in cell culture. Clin Orthop Relat Res 75, 248260 .

Grigolo, B., Lisignoli, G., Piacentini, A., Fiorini, M., Gobbi, P., Mazzotti, G., Duca, M., Pavesio, A., and Facchini, A. (2002). Evidence for redifferentiation of human chondrocytes grown on a hyaluronan-based biomaterial (HYAff 11): molecular, immunohistochemical and ultrastructural analysis. Biomaterials 23, 1187-1195.

Gudas, R., Kalesinskas, R.J., Kimtys, V., Stankevicius, E., Toliusis, V., Bernotavicius, G., and Smailys, A. (2005). A prospective randomized clinical study of mosaic osteochondral autologous transplantation versus microfracture for the treatment of osteochondral defects in the knee joint in young athletes. Arthroscopy 21, 1066-1075

Guerit, D., Philipot, D., Chuchana, P., Toupet, K., Brondello, J.M., Mathieu, M., Jorgensen, C., and Noel, D. (2013). Sox9-Regulated miRNA-574-3p Inhibits Chondrogenic Differentiation of Mesenchymal Stem Cells. PloS one 8 , e62582.

Guilak, F., Lott, K.E., Awad, H.A., Cao, Q., Hicok, K.C., Fermor, B., and Gimble, J.M. (2006). Clonal analysis of the differentiation potential of human adipose-derived adult stem cells. J. Cell. Physiol. 206, 229-237.

Hall, B.K., and Miyake, T. (1995). Divide, accumulate, differentiate: cell condensation in skeletal development revisited. Int. J. Dev. Biol. 39, 881-893.

Handorf, A.M., and Li, W.J. (2011). Fibroblast growth factor-2 primes human mesenchymal stem cells for enhanced chondrogenesis. PloS one 6, e22887.

Handorf, A.M., and Li, W.J. (2014). Induction of mesenchymal stem cell chondrogenesis through sequential administration of growth factors within specific temporal windows. J. Cell. Physiol. 229, 162171 .

Hangody, L., Dobos, J., Balo, E., Panics, G., Hangody, L.R., and Berkes, I. (2010). Clinical experiences with autologous osteochondral mosaicplasty in an athletic population: a 17-year prospective multicenter study. Am. J. Sports Med. 38, 1125-1133. 
Hangody, L., and Fules, P. (2003). Autologous osteochondral mosaicplasty for the treatment of fullthickness defects of weight-bearing joints: ten years of experimental and clinical experience. J. Bone Joint Surg. Am. 85-A Suppl 2, 25-32.

Hangody, L., Kish, G., Karpati, Z., Szerb, I., and Udvarhelyi, I. (1997). Arthroscopic autogenous osteochondral mosaicplasty for the treatment of femoral condylar articular defects. A preliminary report. Knee Surg. Sports Traumatol. Arthrosc. 5, 262-267.

Harris, R.G., Herzog, E.L., Bruscia, E.M., Grove, J.E., Van Arnam, J.S., and Krause, D.S. (2004). Lack of a fusion requirement for development of bone marrow-derived epithelia. Science 305, 90-93.

Hartmann, C., and Tabin, C.J. (2001). Wnt-14 plays a pivotal role in inducing synovial joint formation in the developing appendicular skeleton. Cell 104, 341-351.

Hauselmann, H.J., Fernandes, R.J., Mok, S.S., Schmid, T.M., Block, J.A., Aydelotte, M.B., Kuettner, K.E., and Thonar, E.J. (1994). Phenotypic stability of bovine articular chondrocytes after long-term culture in alginate beads. J. Cell Sci. 107 ( Pt 1), 17-27.

Hayes, A.J., MacPherson, S., Morrison, H., Dowthwaite, G., and Archer, C.W. (2001). The development of articular cartilage: evidence for an appositional growth mechanism. Anat. Embryol. (Berl). 203, 469-479.

He, L., and Hannon, G.J. (2004). MicroRNAs: small RNAs with a big role in gene regulation. Nature reviews. Genetics 5, 522-531.

Hennig, T., Lorenz, H., Thiel, A., Goetzke, K., Dickhut, A., Geiger, F., and Richter, W. (2007). Reduced chondrogenic potential of adipose tissue derived stromal cells correlates with an altered TGFbeta receptor and BMP profile and is overcome by BMP-6. J. Cell. Physiol. 211, 682-691.

Herlofsen, S.R., Bryne, J.C., Hoiby, T., Wang, L., Issner, R., Zhang, X., Coyne, M.J., Boyle, P., Gu, H., Meza-Zepeda, L.A., Collas, P., Mikkelsen, T.S., and Brinchmann, J.E. (2013). Genome-wide map of quantified epigenetic changes during in vitro chondrogenic differentiation of primary human mesenchymal stem cells. BMC genomics 14, 105.

Herlofsen, S.R., Høiby, T., Cacchiarelli, D., Zhang, X., Mikkelsen, T.S., and Brinchmann, J.E. (2014). Importance of SOX8 for in vitro chondrogenic differentiation of human mesenchymal stromal cells. Stem Cells In Press.

Herlofsen, S.R., Kuchler, A.M., Melvik, J.E., and Brinchmann, J.E. (2011). Chondrogenic differentiation of human bone marrow-derived mesenchymal stem cells in self-gelling alginate discs reveals novel chondrogenic signature gene clusters. Tissue Eng Part A 17, 1003-1013.

Hills, G.M. (1940). The metabolism of articular cartilage. Biochem. J. 34, 1070-1077.

Hjelle, K., Solheim, E., Strand, T., Muri, R., and Brittberg, M. (2002). Articular cartilage defects in 1,000 knee arthroscopies. Arthroscopy 18, 730-734.

Hjermundrud, V., Bjune, T.K., Risberg, M.A., Engebretsen, L., and Aroen, A. (2010). Full-thickness cartilage lesion do not affect knee function in patients with ACL injury. Knee Surg. Sports Traumatol. Arthrosc. 18, 298-303.

Hochedlinger, K., and Jaenisch, R. (2006). Nuclear reprogramming and pluripotency. Nature 441, 10611067.

Hoemann, C., Kandel, R., Roberts, S., Saris, D.B.F., Creemers, L., Mainil-Varlet, P., Methot, S., Hollander, A.P., and Buschmann, M.D. (2011). International Cartilage Repair Society (ICRS) Recommended 
Guidelines for Histological Endpoints for Cartilage Repair Studies in Animal Models and Clinical Trials. Cartilage 2, 153-172.

Holterman, C.E., and Rudnicki, M.A. (2005). Molecular regulation of satellite cell function. Semin. Cell Dev. Biol. 16, 575-584.

Holtzer, H., Abbott, J., Lash, J., and Holtzer, S. (1960). The Loss of Phenotypic Traits by Differentiated Cells in Vitro, I. Dedifferentiation of Cartilage Cells. Proc. Natl. Acad. Sci. U. S. A. 46, 1533-1542.

Horwitz, E.M., Gordon, P.L., Koo, W.K., Marx, J.C., Neel, M.D., McNall, R.Y., Muul, L., and Hofmann, T. (2002). Isolated allogeneic bone marrow-derived mesenchymal cells engraft and stimulate growth in children with osteogenesis imperfecta: Implications for cell therapy of bone. Proc. Natl. Acad. Sci. U. S. A. 99, 8932-8937.

Horwitz, E.M., Le Blanc, K., Dominici, M., Mueller, I., Slaper-Cortenbach, I., Marini, F.C., Deans, R.J., Krause, D.S., and Keating, A. (2005). Clarification of the nomenclature for MSC: The International Society for Cellular Therapy position statement. Cytotherapy 7, 393-395.

Huang, K., Shen, Y., Xue, Z., Bibikova, M., April, C., Liu, Z., Cheng, L., Nagy, A., Pellegrini, M., Fan, J.B., and Fan, G. (2013). A Panel of CpG Methylation Sites Distinguishes Human Embryonic Stem Cells and Induced Pluripotent Stem Cells. Stem cell reports.

Huang, W., Chung, U.I., Kronenberg, H.M., and de Crombrugghe, B. (2001). The chondrogenic transcription factor Sox 9 is a target of signaling by the parathyroid hormone-related peptide in the growth plate of endochondral bones. Proc. Natl. Acad. Sci. U. S. A. 98, 160-165.

Hunter, W. (1742). Of the Structure and Diseases of Articulating Cartilages, by William Hunter, Surgeon. Philosophical Transactions of the Royal Society of London 42, 514-521.

Hunziker, E.B. (2002a). Articular cartilage repair: basic science and clinical progress. A review of the current status and prospects. Osteoarthritis Cartilage 10, 432-463.

Hunziker, E.B., Quinn, T.M., and Hauselmann, H.J. (2002b). Quantitative structural organization of normal adult human articular cartilage. Osteoarthritis Cartilage 10, 564-572.

Hurtig, M.B., Buschmann, M.D., Fortier, L.A., Hoemann, C.D., Hunziker, E.B., Jurvelin, J.S., MainilVarlet, P., McIlwraith, C.W., Sah, R.L., and Whiteside, R.A. (2011). Preclinical Studies for Cartilage Repair: Recommendations from the International Cartilage Repair Society. Cartilage 2, 137-152.

Hyllested, J.L., Veje, K., and Ostergaard, K. (2002). Histochemical studies of the extracellular matrix of human articular cartilage--a review. Osteoarthritis Cartilage 10, 333-343.

ICRS. (2000). ICRS Cartilage Injury Evaluation Package, ed. M. Brittberg.

Iliopoulos, D., Malizos, K.N., Oikonomou, P., and Tsezou, A. (2008). Integrative microRNA and proteomic approaches identify novel osteoarthritis genes and their collaborative metabolic and inflammatory networks. PloS one 3, e3740.

Im, G.I., Lee, J.M., and Kim, H.J. (2011). Wnt inhibitors enhance chondrogenesis of human mesenchymal stem cells in a long-term pellet culture. Biotechnology letters 33, 1061-1068.

Im, G.I., Shin, Y.W., and Lee, K.B. (2005). Do adipose tissue-derived mesenchymal stem cells have the same osteogenic and chondrogenic potential as bone marrow-derived cells? Osteoarthritis Cartilage 13, 845-853. 
Iwamoto, M., Higuchi, Y., Koyama, E., Enomoto-Iwamoto, M., Kurisu, K., Yeh, H., Abrams, W.R., Rosenbloom, J., and Pacifici, M. (2000). Transcription factor ERG variants and functional diversification of chondrocytes during limb long bone development. J. Cell Biol. 150, 27-40.

Iwamoto, M., Tamamura, Y., Koyama, E., Komori, T., Takeshita, N., Williams, J.A., Nakamura, T., Enomoto-Iwamoto, M., and Pacifici, M. (2007). Transcription factor ERG and joint and articular cartilage formation during mouse limb and spine skeletogenesis. Dev. Biol. 305, 40-51.

Jaenisch, R. (2004). Human cloning - the science and ethics of nuclear transplantation. N. Engl. J. Med. $351,2787-2791$.

Jaenisch, R., and Meissner, A. (2006). Politically correct human embryonic stem cells? N. Engl. J. Med. 354, 1208-1209; author reply 1208-1209.

Jakobsen, R.B., and Engebretsen, L. (2007). An analysis of the quality of cartilage repair studies - An Update. ISAKOS CURRENT CONCEPTS.

Jakobsen, R.B., Engebretsen, L., and Slauterbeck, J.R. (2005). An analysis of the quality of cartilage repair studies. J. Bone Joint Surg. Am. 87, 2232-2239.

Jansen, E.J., Emans, P.J., Douw, C.M., Guldemond, N.A., Van Rhijn, L.W., Bulstra, S.K., and Kuijer, R. (2008). One intra-articular injection of hyaluronan prevents cell death and improves cell metabolism in a model of injured articular cartilage in the rabbit. J. Orthop. Res. 26, 624-630.

Janssen, H.L., Reesink, H.W., Lawitz, E.J., Zeuzem, S., Rodriguez-Torres, M., Patel, K., van der Meer, A.J., Patick, A.K., Chen, A., Zhou, Y., Persson, R., King, B.D., Kauppinen, S., Levin, A.A., and Hodges, M.R. (2013). Treatment of HCV Infection by Targeting MicroRNA. N. Engl. J. Med.

Jia, S., Liu, L., Pan, W., Meng, G., Duan, C., Zhang, L., Xiong, Z., and Liu, J. (2012). Oriented cartilage extracellular matrix-derived scaffold for cartilage tissue engineering. J Biosci Bioeng 113, 647-653.

Jiang, Y., Henderson, D., Blackstad, M., Chen, A., Miller, R.F., and Verfaillie, C.M. (2003).

Neuroectodermal differentiation from mouse multipotent adult progenitor cells. Proc. Natl. Acad. Sci. U. S. A. 100 Suppl 1, 11854-11860.

Jiang, Y., Jahagirdar, B.N., Reinhardt, R.L., Schwartz, R.E., Keene, C.D., Ortiz-Gonzalez, X.R., Reyes, M., Lenvik, T., Lund, T., Blackstad, M., Du, J., Aldrich, S., Lisberg, A., Low, W.C., Largaespada, D.A., and Verfaillie, C.M. (2002). Pluripotency of mesenchymal stem cells derived from adult marrow. Nature 418, 41-49.

Johnson, K., Zhu, S., Tremblay, M.S., Payette, J.N., Wang, J., Bouchez, L.C., Meeusen, S., Althage, A., Cho, C.Y., Wu, X., and Schultz, P.G. (2012). A Stem Cell-Based Approach to Cartilage Repair. Science.

Johnstone, B., Hering, T.M., Caplan, A.I., Goldberg, V.M., and Yoo, J.U. (1998). In vitro chondrogenesis of bone marrow-derived mesenchymal progenitor cells. Exp. Cell Res. 238, 265-272.

Joseph, N.M., and Morrison, S.J. (2005). Toward an understanding of the physiological function of Mammalian stem cells. Developmental cell 9, 173-183.

Kanaan, R.A., and Kanaan, L.A. (2006). Transforming growth factor beta1, bone connection. Med Sci Monit 12, RA164-169.

Kardassis, D., Murphy, C., Fotsis, T., Moustakas, A., and Stournaras, C. (2009). Control of transforming growth factor beta signal transduction by small GTPases. The FEBS journal 276, 2947-2965. 
Karlsen, T.A., and Brinchmann, J.E. (2013). Liposome delivery of microRNA-145 to mesenchymal stem cells leads to immunological off-target effects mediated by RIG-I. Mol Ther 21, 1169-1181.

Karlsen, T.A., Shahdadfar, A., and Brinchmann, J.E. (2011). Human primary articular chondrocytes, chondroblasts-like cells, and dedifferentiated chondrocytes: differences in gene, microRNA, and protein expression and phenotype. Tissue engineering. Part C, Methods 17, 219-227.

Kaviani, A., Perry, T.E., Dzakovic, A., Jennings, R.W., Ziegler, M.M., and Fauza, D.O. (2001). The amniotic fluid as a source of cells for fetal tissue engineering. J. Pediatr. Surg. 36, 1662-1665.

Kayakabe, M., Tsutsumi, S., Watanabe, H., Kato, Y., and Takagishi, K. (2006). Transplantation of autologous rabbit BM-derived mesenchymal stromal cells embedded in hyaluronic acid gel sponge into osteochondral defects of the knee. Cytotherapy 8, 343-353.

Keller, B., Yang, T., Chen, Y., Munivez, E., Bertin, T., Zabel, B., and Lee, B. (2011). Interaction of TGFbeta and BMP signaling pathways during chondrogenesis. PloS one 6, e16421.

Kim, D., Song, J., and Jin, E.J. (2010a). MicroRNA-221 regulates chondrogenic differentiation through promoting proteosomal degradation of slug by targeting Mdm2. J. Biol. Chem. 285, 26900-26907.

Kim, H.J., and Im, G.I. (2010b). The effects of ERK1/2 inhibitor on the chondrogenesis of bone marrowand adipose tissue-derived multipotent mesenchymal stromal cells. Tissue Eng Part A 16, 851-860.

Kim, T.K., and Eberwine, J.H. (2010c). Mammalian cell transfection: the present and the future. Analytical and bioanalytical chemistry $397,3173-3178$.

Kim, Y.J., Kim, H.J., and Im, G.I. (2008). PTHrP promotes chondrogenesis and suppresses hypertrophy from both bone marrow-derived and adipose tissue-derived MSCs. Biochem. Biophys. Res. Commun. 373, 104-108.

Kist, R., Schrewe, H., Balling, R., and Scherer, G. (2002). Conditional inactivation of Sox9: a mouse model for campomelic dysplasia. Genesis 32, 121-123.

Knudson, C.B., and Knudson, W. (2001). Cartilage proteoglycans. Semin. Cell Dev. Biol. 12, 69-78.

Knudson, W., and Loeser, R.F. (2002). CD44 and integrin matrix receptors participate in cartilage homeostasis. Cell. Mol. Life Sci. 59, 36-44.

Knutsen, G., Drogset, J.O., Engebretsen, L., Grontvedt, T., Isaksen, V., Ludvigsen, T.C., Roberts, S., Solheim, E., Strand, T., and Johansen, O. (2007). A randomized trial comparing autologous chondrocyte implantation with microfracture. Findings at five years. J. Bone Joint Surg. Am. 89, 2105-2112.

Knutsen, G., Engebretsen, L., Ludvigsen, T.C., Drogset, J.O., Grontvedt, T., Solheim, E., Strand, T., Roberts, S., Isaksen, V., and Johansen, O. (2004). Autologous chondrocyte implantation compared with microfracture in the knee. A randomized trial. Journal of Bone \& Joint Surgery - American Volume $86-A$, 455-464.

Kobayashi, N., Uemura, H., Nagahama, K., Okudela, K., Furuya, M., Ino, Y., Ito, Y., Hirano, H., Inayama, Y., Aoki, I., Nagashima, Y., Kubota, Y., and Ishiguro, H. (2012). Identification of miR-30d as a novel prognostic maker of prostate cancer. Oncotarget 3, 1455-1471.

Kobayashi, T., Lu, J., Cobb, B.S., Rodda, S.J., McMahon, A.P., Schipani, E., Merkenschlager, M., and Kronenberg, H.M. (2008). Dicer-dependent pathways regulate chondrocyte proliferation and differentiation. Proc. Natl. Acad. Sci. U. S. A. 105, 1949-1954. 
Koch, T.G., Berg, L.C., and Betts, D.H. (2008). Concepts for the clinical use of stem cells in equine medicine. Can. Vet. J. 49, 1009-1017.

Koga, H., Muneta, T., Ju, Y.J., Nagase, T., Nimura, A., Mochizuki, T., Ichinose, S., von der Mark, K., and Sekiya, I. (2007). Synovial stem cells are regionally specified according to local microenvironments after implantation for cartilage regeneration. Stem Cells 25, 689-696.

Koga, H., Shimaya, M., Muneta, T., Nimura, A., Morito, T., Hayashi, M., Suzuki, S., Ju, Y.J., Mochizuki, T., and Sekiya, I. (2008). Local adherent technique for transplanting mesenchymal stem cells as a potential treatment of cartilage defect. Arthritis Res Ther 10, R84.

Kogler, G., Sensken, S., Airey, J.A., Trapp, T., Muschen, M., Feldhahn, N., Liedtke, S., Sorg, R.V., Fischer, J., Rosenbaum, C., Greschat, S., Knipper, A., Bender, J., Degistirici, O., Gao, J., Caplan, A.I., Colletti, E.J., Almeida-Porada, G., Muller, H.W., Zanjani, E., and Wernet, P. (2004). A new human somatic stem cell from placental cord blood with intrinsic pluripotent differentiation potential. J. Exp. Med. 200, 123-135.

Kucia, M., Reca, R., Campbell, F.R., Zuba-Surma, E., Majka, M., Ratajczak, J., and Ratajczak, M.Z. (2006). A population of very small embryonic-like (VSEL) CXCR4(+)SSEA-1(+)Oct-4+ stem cells identified in adult bone marrow. Leukemia 20, 857-869.

Kuroda, R., Ishida, K., Matsumoto, T., Akisue, T., Fujioka, H., Mizuno, K., Ohgushi, H., Wakitani, S., and Kurosaka, M. (2007). Treatment of a full-thickness articular cartilage defect in the femoral condyle of an athlete with autologous bone-marrow stromal cells. Osteoarthritis Cartilage 15, 226-231.

Kwan, K.M., Pang, M.K., Zhou, S., Cowan, S.K., Kong, R.Y., Pfordte, T., Olsen, B.R., Sillence, D.O., Tam, P.P., and Cheah, K.S. (1997). Abnormal compartmentalization of cartilage matrix components in mice lacking collagen X: implications for function. J. Cell Biol. 136, 459-471.

Lakshmipathy, U., and Verfaillie, C. (2005). Stem cell plasticity. Blood Rev. 19, 29-38.

Lanford, R.E., Hildebrandt-Eriksen, E.S., Petri, A., Persson, R., Lindow, M., Munk, M.E., Kauppinen, S., and Orum, H. (2010). Therapeutic silencing of microRNA-122 in primates with chronic hepatitis $\mathrm{C}$ virus infection. Science 327, 198-201.

Le Blanc, K., Rasmusson, I., Sundberg, B., Gotherstrom, C., Hassan, M., Uzunel, M., and Ringden, O. (2004). Treatment of severe acute graft-versus-host disease with third party haploidentical mesenchymal stem cells. Lancet 363, 1439-1441.

Lee, A.S., Ellman, M.B., Yan, D., Kroin, J.S., Cole, B.J., van Wijnen, A.J., and Im, H.J. (2013). A current review of molecular mechanisms regarding osteoarthritis and pain. Gene 527, 440-447.

Lee, R.H., Kim, B., Choi, I., Kim, H., Choi, H.S., Suh, K., Bae, Y.C., and Jung, J.S. (2004).

Characterization and expression analysis of mesenchymal stem cells from human bone marrow and adipose tissue. Cell. Physiol. Biochem. 14, 311-324.

Lefebvre, V., Behringer, R.R., and de Crombrugghe, B. (2001). L-Sox5, Sox6 and Sox9 control essential steps of the chondrocyte differentiation pathway. Osteoarthritis Cartilage 9 Suppl A, S69-75.

Li, J., Ezzelarab, M.B., and Cooper, D.K. (2012). Do mesenchymal stem cells function across species barriers? Relevance for xenotransplantation. Xenotransplantation 19, 273-285.

Li, L. (2010). Dimension reduction for high-dimensional data. Methods Mol. Biol. 620, 417-434.

Li, L., and Xie, T. (2005). Stem cell niche: structure and function. Annu. Rev. Cell Dev. Biol. 21, 605-631.

Li, R. (2013). The art of choreographing asymmetric cell division. Developmental cell 25, 439-450. 
Lin, C.C., Liu, L.Z., Addison, J.B., Wonderlin, W.F., Ivanov, A.V., and Ruppert, J.M. (2011). A KLF4miRNA-206 autoregulatory feedback loop can promote or inhibit protein translation depending upon cell context. Mol. Cell. Biol. 31, 2513-2527.

Lin, H. (2002). The stem-cell niche theory: lessons from flies. Nature reviews. Genetics 3, 931-940.

Lin, X., Wu, L., Zhang, Z., Yang, R., Guan, Q., Hou, X., and Wu, Q. (2013). MiR-335-5p Promotes Chondrogenesis in Mouse Mesenchymal Stem Cells and is Regulated Through Two Positive Feedback Loops. J. Bone Miner. Res.

Liu, Z., Lavine, K.J., Hung, I.H., and Ornitz, D.M. (2007). FGF18 is required for early chondrocyte proliferation, hypertrophy and vascular invasion of the growth plate. Dev. Biol. 302, 80-91.

Loken, S., Jakobsen, R.B., Aroen, A., Heir, S., Shahdadfar, A., Brinchmann, J.E., Engebretsen, L., and Reinholt, F.P. (2008). Bone marrow mesenchymal stem cells in a hyaluronan scaffold for treatment of an osteochondral defect in a rabbit model. Knee Surg. Sports Traumatol. Arthrosc. 16, 896-903.

Lorentzon, R., Alfredson, H., and Hildingsson, C. (1998). Treatment of deep cartilage defects of the patella with periosteal transplantation. Knee Surg. Sports Traumatol. Arthrosc. 6, 202-208.

Lorenzo, P., Bayliss, M.T., and Heinegard, D. (2004). Altered patterns and synthesis of extracellular matrix macromolecules in early osteoarthritis. Matrix Biol. 23, 381-391.

Luo, W., Guo, C., Zheng, J., Chen, T.L., Wang, P.Y., Vertel, B.M., and Tanzer, M.L. (2000). Aggrecan from start to finish. J. Bone Miner. Metab. 18, 51-56.

Ma, H.L., Hung, S.C., Lin, S.Y., Chen, Y.L., and Lo, W.H. (2003). Chondrogenesis of human mesenchymal stem cells encapsulated in alginate beads. Journal of biomedical materials research. Part A 64, 273-281.

Ma, K., Titan, A.L., Stafford, M., Zheng, C., and Levenston, M.E. (2012). Variations in chondrogenesis of human bone marrow-derived mesenchymal stem cells in fibrin/alginate blended hydrogels. Acta biomaterialia $8,3754-3764$.

Mainil-Varlet, P., Aigner, T., Brittberg, M., Bullough, P., Hollander, A., Hunziker, E., Kandel, R., Nehrer, S., Pritzker, K., Roberts, S., Stauffer, E., and International Cartilage Repair, S. (2003). Histological assessment of cartilage repair: a report by the Histology Endpoint Committee of the International Cartilage Repair Society (ICRS). J. Bone Joint Surg. Am. 85-A Suppl 2, 45-57.

Malkov, V.A., Serikawa, K.A., Balantac, N., Watters, J., Geiss, G., Mashadi-Hossein, A., and Fare, T. (2009). Multiplexed measurements of gene signatures in different analytes using the Nanostring nCounter Assay System. BMC research notes 2, 80 .

Manning, W.K., and Bonner, W.M., Jr. (1967). Isolation and culture of chondrocytes from human adult articular cartilage. Arthritis Rheum. 10, 235-239.

Marcacci, M., Berruto, M., Brocchetta, D., Delcogliano, A., Ghinelli, D., Gobbi, A., Kon, E., Pederzini, L., Rosa, D., Sacchetti, G.L., Stefani, G., and Zanasi, S. (2005). Articular cartilage engineering with Hyalograft C: 3-year clinical results. Clin Orthop Relat Res 435, 96-105.

Martinez-Sanchez, A., Dudek, K.A., and Murphy, C.L. (2012). Regulation of human chondrocyte function through direct inhibition of cartilage master regulator SOX9 by microRNA-145 (miRNA-145). J. Biol. Chem. 287, 916-924.

Massague, J., and Gomis, R.R. (2006). The logic of TGFbeta signaling. FEBS Lett. 580, 2811-2820. 
Mastbergen, S.C., Saris, D.B., and Lafeber, F.P. (2013). Functional articular cartilage repair: here, near, or is the best approach not yet clear? Nature reviews. Rheumatology 9, 277-290.

Matricali, G.A., Dereymaeker, G.P., and Luyten, F.P. (2010). Donor site morbidity after articular cartilage repair procedures: a review. Acta Orthop. Belg. 76, 669-674.

Matsumoto, T., Okabe, T., Ikawa, T., Iida, T., Yasuda, H., Nakamura, H., and Wakitani, S. (2010).

Articular cartilage repair with autologous bone marrow mesenchymal cells. J. Cell. Physiol. 225, 291-295.

Matsusue, Y., Yamamuro, T., and Hama, H. (1993). Arthroscopic multiple osteochondral transplantation to the chondral defect in the knee associated with anterior cruciate ligament disruption. Arthroscopy 9, 318321.

Mayan, M.D., Carpintero-Fernandez, P., Gago-Fuentes, R., Martinez-de-Ilarduya, O., Wang, H.Z., Valiunas, V., Brink, P., and Blanco, F.J. (2013). Human articular chondrocytes express multiple gap junction proteins: differential expression of connexins in normal and osteoarthritic cartilage. Am. J. Pathol. 182, 1337-1346.

McAlinden, A., Johnstone, B., Kollar, J., Kazmi, N., and Hering, T.M. (2008). Expression of two novel alternatively spliced COL2A1 isoforms during chondrocyte differentiation. Matrix Biol. 27, 254-266.

McCoy, B., and Miniaci, A. (2012). Osteochondral autograft transplantation/mosaicplasty. J Knee Surg 25, 99-108.

Medvinsky, A., and Smith, A. (2003). Stem cells: Fusion brings down barriers. Nature 422, 823-825.

Meijer, H.A., Kong, Y.W., Lu, W.T., Wilczynska, A., Spriggs, R.V., Robinson, S.W., Godfrey, J.D., Willis, A.E., and Bushell, M. (2013). Translational repression and eIF4A2 activity are critical for microRNAmediated gene regulation. Science $340,82-85$.

Melville, H., Carpiniello, M., Hollis, K., Staffaroni, A., and Golestaneh, N. (2013). Stem cells: a new paradigm for disease modeling and developing therapies for age-related macular degeneration. J Transl Med 11, 53.

Messner, K., and Maletius, W. (1996). The long-term prognosis for severe damage to weight-bearing cartilage in the knee: a 14-year clinical and radiographic follow-up in 28 young athletes. Acta Orthop. Scand. 67, 165-168.

Miao, Z., Jin, J., Chen, L., Zhu, J., Huang, W., Zhao, J., Qian, H., and Zhang, X. (2006). Isolation of mesenchymal stem cells from human placenta: comparison with human bone marrow mesenchymal stem cells. Cell Biol. Int. 30, 681-687.

Mikkelsen, T.S., Xu, Z., Zhang, X., Wang, L., Gimble, J.M., Lander, E.S., and Rosen, E.D. (2010). Comparative epigenomic analysis of murine and human adipogenesis. Cell 143, 156-169.

Mithoefer, K., Hambly, K., Logerstedt, D., Ricci, M., Silvers, H., and Della Villa, S. (2012). Current concepts for rehabilitation and return to sport after knee articular cartilage repair in the athlete. J. Orthop. Sports Phys. Ther. 42, 254-273.

Miyaki, S., and Asahara, H. (2012). Macro view of microRNA function in osteoarthritis. Nature reviews. Rheumatology 8, 543-552.

Miyaki, S., Nakasa, T., Otsuki, S., Grogan, S.P., Higashiyama, R., Inoue, A., Kato, Y., Sato, T., Lotz, M.K., and Asahara, H. (2009). MicroRNA-140 is expressed in differentiated human articular chondrocytes and modulates interleukin-1 responses. Arthritis Rheum. 60, 2723-2730. 
Miyaki, S., Sato, T., Inoue, A., Otsuki, S., Ito, Y., Yokoyama, S., Kato, Y., Takemoto, F., Nakasa, T., Yamashita, S., Takada, S., Lotz, M.K., Ueno-Kudo, H., and Asahara, H. (2010). MicroRNA-140 plays dual roles in both cartilage development and homeostasis. Genes Dev. 24, 1173-1185.

Miyakoshi, N., Kobayashi, M., Nozaka, K., Okada, K., Shimada, Y., and Itoi, E. (2005). Effects of intraarticular administration of basic fibroblast growth factor with hyaluronic acid on osteochondral defects of the knee in rabbits. Arch. Orthop. Trauma Surg. 125, 683-692.

Miyanishi, M., Mori, Y., Seita, J., Chen, J.Y., Karten, S., Chan, C.K., Nakauchi, H., and Weissman, I.L. (2013). Do pluripotent stem cells exist in adult mice as very small embryonic stem cells? Stem cell reports $1,198-208$.

Mont, M.A., Baumgarten, K.M., Rifai, A., Bluemke, D.A., Jones, L.C., and Hungerford, D.S. (2000). Atraumatic osteonecrosis of the knee. J. Bone Joint Surg. Am. 82, 1279-1290.

Moore, K.A., and Lemischka, I.R. (2006). Stem cells and their niches. Science 311, 1880-1885.

Moseley, J.B., O'Malley, K., Petersen, N.J., Menke, T.J., Brody, B.A., Kuykendall, D.H., Hollingsworth, J.C., Ashton, C.M., and Wray, N.P. (2002). A controlled trial of arthroscopic surgery for osteoarthritis of the knee. N. Engl. J. Med. 347, 81-88.

Motulsky, H. (2010). Intuitive Biostatistics - A Nonmathematical Guide to Statistical Thinking. Oxford University Press: New York.

Nakagawa, T., Lee, S.Y., and Reddi, A.H. (2009). Induction of chondrogenesis from human embryonic stem cells without embryoid body formation by bone morphogenetic protein 7 and transforming growth factor beta1. Arthritis Rheum. 60, 3686-3692.

Nakamae, A., Engebretsen, L., Bahr, R., Krosshaug, T., and Ochi, M. (2006). Natural history of bone bruises after acute knee injury: clinical outcome and histopathological findings. Knee Surg. Sports Traumatol. Arthrosc. 14, 1252-1258.

Nakamura, Y., He, X., Kato, H., Wakitani, S., Kobayashi, T., Watanabe, S., Iida, A., Tahara, H., Warman, M.L., Watanapokasin, R., and Postlethwait, J.H. (2012). Sox9 is upstream of microRNA-140 in cartilage. Appl. Biochem. Biotechnol. 166, 64-71.

Nakamura, Y., Inloes, J.B., Katagiri, T., and Kobayashi, T. (2011). Chondrocyte-specific microRNA-140 regulates endochondral bone development and targets Dnpep to modulate bone morphogenetic protein signaling. Mol. Cell. Biol. 31, 3019-3028.

NatureNews. (2007). Stem cells: The hard copy. Nature 446, 485-486.

Nicolas, F.E. (2011a). Experimental validation of microRNA targets using a luciferase reporter system. Methods Mol. Biol. 732, 139-152.

Nicolas, F.E., Pais, H., Schwach, F., Lindow, M., Kauppinen, S., Moulton, V., and Dalmay, T. (2008). Experimental identification of microRNA-140 targets by silencing and overexpressing miR-140. RNA 14, $2513-2520$

Nicolas, F.E., Pais, H., Schwach, F., Lindow, M., Kauppinen, S., Moulton, V., and Dalmay, T. (2011b). mRNA expression profiling reveals conserved and non-conserved miR-140 targets. RNA biology $8,607-$ 615 .

Nieminen, M.T., Nissi, M.J., Mattila, L., and Kiviranta, I. (2012). Evaluation of chondral repair using quantitative MRI. J. Magn. Reson. Imaging 36, 1287-1299. 
O'Driscoll, S.W., and Salter, R.B. (1984). The induction of neochondrogenesis in free intra-articular periosteal autografts under the influence of continuous passive motion. An experimental investigation in the rabbit. J. Bone Joint Surg. Am. 66, 1248-1257.

Okita, K., Ichisaka, T., and Yamanaka, S. (2007). Generation of germline-competent induced pluripotent stem cells. Nature 448, 313-317.

Olsen, B.R., Reginato, A.M., and Wang, W. (2000). Bone development. Annu. Rev. Cell Dev. Biol. 16, 191-220.

Opasanon, S., Muangman, P., and Namviriyachote, N. (2010). Clinical effectiveness of alginate silver dressing in outpatient management of partial-thickness burns. Int Wound J 7, 467-471.

Orom, U.A., Nielsen, F.C., and Lund, A.H. (2008). MicroRNA-10a binds the 5'UTR of ribosomal protein mRNAs and enhances their translation. Mol. Cell 30, 460-471.

Oshima, Y., Watanabe, N., Matsuda, K., Takai, S., Kawata, M., and Kubo, T. (2005). Behavior of transplanted bone marrow-derived GFP mesenchymal cells in osteochondral defect as a simulation of autologous transplantation. J. Histochem. Cytochem. 53, 207-216.

Ostrup, E., Bauersachs, S., Blum, H., Wolf, E., and Hyttel, P. (2010). Differential endometrial gene expression in pregnant and nonpregnant sows. Biol. Reprod. 83, 277-285.

Outerbridge, R.E. (1961). The etiology of chondromalacia patellae. J. Bone Joint Surg. Br. 43-B, 752-757.

Ouyang, L., Liu, P., Yang, S., Ye, S., Xu, W., and Liu, X. (2013). A three-plasma miRNA signature serves as novel biomarkers for osteosarcoma. Med. Oncol. 30, 340.

Pais, H., Nicolas, F.E., Soond, S.M., Swingler, T.E., Clark, I.M., Chantry, A., Moulton, V., and Dalmay, T. (2010). Analyzing mRNA expression identifies Smad3 as a microRNA-140 target regulated only at protein level. RNA 16, 489-494.

Palmer, A.J., Brown, C.P., McNally, E.G., Price, A.J., Tracey, I., Jezzard, P., Carr, A.J., and Glyn-Jones, S. (2013). Non-invasive imaging of cartilage in early osteoarthritis. Bone Joint J 95-B, 738-746.

Patel, D.V., Breazeale, N.M., Behr, C.T., Warren, R.F., Wickiewicz, T.L., and O'Brien, S.J. (1998).

Osteonecrosis of the knee: current clinical concepts. Knee Surg. Sports Traumatol. Arthrosc. 6, 2-11.

Pelttari, K., Winter, A., Steck, E., Goetzke, K., Hennig, T., Ochs, B.G., Aigner, T., and Richter, W. (2006). Premature induction of hypertrophy during in vitro chondrogenesis of human mesenchymal stem cells correlates with calcification and vascular invasion after ectopic transplantation in SCID mice. Arthritis Rheum. 54, 3254-3266.

Phinney, D.G., and Sensebe, L. (2013). Mesenchymal stromal cells: misconceptions and evolving concepts. Cytotherapy 15, 140-145.

Pittenger, M.F., Mackay, A.M., Beck, S.C., Jaiswal, R.K., Douglas, R., Mosca, J.D., Moorman, M.A., Simonetti, D.W., Craig, S., and Marshak, D.R. (1999). Multilineage potential of adult human mesenchymal stem cells. Science 284, 143-147.

Poole, A.R., Kojima, T., Yasuda, T., Mwale, F., Kobayashi, M., and Laverty, S. (2001). Composition and structure of articular cartilage: a template for tissue repair. Clin Orthop Relat Res 391 Suppl, S26-33.

Poole, C.A. (1997). Articular cartilage chondrons: form, function and failure. J. Anat. 191 ( Pt 1), 1-13. 
Radice, M., Brun, P., Cortivo, R., Scapinelli, R., Battaliard, C., and Abatangelo, G. (2000). Hyaluronanbased biopolymers as delivery vehicles for bone-marrow-derived mesenchymal progenitors. J. Biomed. Mater. Res. 50, 101-109.

Retting, K.N., Song, B., Yoon, B.S., and Lyons, K.M. (2009). BMP canonical Smad signaling through Smad1 and Smad5 is required for endochondral bone formation. Development 136, 1093-1104.

Reyes, M., Lund, T., Lenvik, T., Aguiar, D., Koodie, L., and Verfaillie, C.M. (2001). Purification and ex vivo expansion of postnatal human marrow mesodermal progenitor cells. Blood 98, 2615-2625.

Ringe, J., Burmester, G.R., and Sittinger, M. (2012). Regenerative medicine in rheumatic disease-progress in tissue engineering. Nature reviews. Rheumatology 8, 493-498.

Roberts, S., Menage, J., Sandell, L.J., Evans, E.H., and Richardson, J.B. (2009). Immunohistochemical study of collagen types I and II and procollagen IIA in human cartilage repair tissue following autologous chondrocyte implantation. The Knee 16, 398-404.

Robinton, D.A., and Daley, G.Q. (2012). The promise of induced pluripotent stem cells in research and therapy. Nature 481, 295-305.

Ross, M.H., Pawlina, W., and Kaye, G.I. (2003). Histology: a text and atlas. Lippincott Williams \& Wilkins: Baltimore.

Rothwell, A.G., and Bentley, G. (1973). Chondrocyte multiplication in osteoarthritic articular cartilage. J. Bone Joint Surg. Br. 55, 588-594.

Ryan, J.M., Barry, F.P., Murphy, J.M., and Mahon, B.P. (2005). Mesenchymal stem cells avoid allogeneic rejection. J Inflamm (Lond) 2, 8.

Sadler, T.W., Langman, J., and Ecker, P.M. (2006). Langman's medical embryology. Lippincott Williams \& Wilkins: Philadelphia.

Saris, D., Price, A., Drogset, J.O., Podskubka, A., Tsuchida, A., Bezuidenhoudt, M., Kili, S., Levine, D.W., and Brittberg, M. (2013). SUMMIT Prospective, Randomized, Controlled Trial: Response Rates To Matrix-induced Autologous Chondrocyte Implant (MACI) Versus Microfracture (MFX) By Lesion Characteristics. Orthopaedic Journal of Sports Medicine 1 .

Saris, D.B., Vanlauwe, J., Victor, J., Almqvist, K.F., Verdonk, R., Bellemans, J., Luyten, F.P., Tig/Act, and Group, E.X.T.S. (2009). Treatment of symptomatic cartilage defects of the knee: characterized chondrocyte implantation results in better clinical outcome at 36 months in a randomized trial compared to microfracture. Am. J. Sports Med. 37 Suppl 1, 10S-19S.

Saris, D.B., Vanlauwe, J., Victor, J., Haspl, M., Bohnsack, M., Fortems, Y., Vandekerckhove, B., Almqvist, K.F., Claes, T., Handelberg, F., Lagae, K., van der Bauwhede, J., Vandenneucker, H., Yang, K.G., Jelic, M., Verdonk, R., Veulemans, N., Bellemans, J., and Luyten, F.P. (2008). Characterized chondrocyte implantation results in better structural repair when treating symptomatic cartilage defects of the knee in a randomized controlled trial versus microfracture. Am. J. Sports Med. 36, 235-246.

Schallmoser, K., and Strunk, D. (2009). Preparation of pooled human platelet lysate (pHPL) as an efficient supplement for animal serum-free human stem cell cultures. Journal of visualized experiments : JoVE.

Schenck, R.C., Jr., and Goodnight, J.M. (1996). Osteochondritis dissecans. J. Bone Joint Surg. Am. 78, 439-456.

Schnabel, L.V., Fortier, L.A., McIlwraith, C.W., and Nobert, K.M. (2013). Therapeutic use of stem cells in horses: which type, how, and when? Vet. J. 197, 570-577. 
Schwanhausser, B., Busse, D., Li, N., Dittmar, G., Schuchhardt, J., Wolf, J., Chen, W., and Selbach, M. (2011). Global quantification of mammalian gene expression control. Nature 473, 337-342.

Schwartz, S.D., Hubschman, J.-P., Heilwell, G., Franco-Cardenas, V., Pan, C.K., Ostrick, R.M., Mickunas, E., Gay, R., Klimanskaya, I., and Lanza, R. (2012). Embryonic stem cell trials for macular degeneration: a preliminary report. The Lancet $379,713-720$.

Seibel, M.J., Robins, S.P., and Bilezikian, J.P. (2006). Dynamics of bone and cartilage metabolism. Academic Press: San Diego.

Sekiya, I., Colter, D.C., and Prockop, D.J. (2001). BMP-6 enhances chondrogenesis in a subpopulation of human marrow stromal cells. Biochem. Biophys. Res. Commun. 284, 411-418.

Sekiya, I., Larson, B.L., Vuoristo, J.T., Reger, R.L., and Prockop, D.J. (2005). Comparison of effect of BMP-2, -4 , and -6 on in vitro cartilage formation of human adult stem cells from bone marrow stroma. Cell Tissue Res. 320, 269-276.

Seo, M.J., Suh, S.Y., Bae, Y.C., and Jung, J.S. (2005). Differentiation of human adipose stromal cells into hepatic lineage in vitro and in vivo. Biochem. Biophys. Res. Commun. 328, 258-264.

Serafini, M., and Verfaillie, C.M. (2006). Pluripotency in adult stem cells: state of the art. Seminars in reproductive medicine $24,379-388$.

Shahdadfar, A., Loken, S., Dahl, J.A., Tunheim, S.H., Collas, P., Reinholt, F.P., Engebretsen, L., and Brinchmann, J.E. (2008). Persistence of collagen type II synthesis and secretion in rapidly proliferating human articular chondrocytes in vitro. Tissue Eng Part A 14, 1999-2007.

Shelbourne, K.D., Jari, S., and Gray, T. (2003). Outcome of untreated traumatic articular cartilage defects of the knee: a natural history study. J. Bone Joint Surg. Am. 85-A Suppl 2, 8-16.

Shellock, F.G., Hiller, W.D., Ainge, G.R., Brown, D.W., and Dierenfield, L. (2003). Knees of Ironman triathletes: magnetic resonance imaging assessment of older ( $>35$ years old) competitors. J. Magn. Reson. Imaging 17, 122-130.

Shi, S., and Gronthos, S. (2003). Perivascular niche of postnatal mesenchymal stem cells in human bone marrow and dental pulp. J. Bone Miner. Res. 18, 696-704.

Shoji, T., Nakasa, T., Yamasaki, K., Kodama, A., Miyaki, S., Niimoto, T., Okuhara, A., Kamei, N., Adachi, N., and Ochi, M. (2012). The effect of intra-articular injection of microRNA-210 on ligament healing in a rat model. Am. J. Sports Med. 40, 2470-2478.

Shum, L., and Nuckolls, G. (2002). The life cycle of chondrocytes in the developing skeleton. Arthritis research 4, 94-106.

Smidsrod, O., and Skjak-Braek, G. (1990). Alginate as immobilization matrix for cells. Trends Biotechnol. $8,71-78$.

Smits, P., Li, P., Mandel, J., Zhang, Z., Deng, J.M., Behringer, R.R., de Crombrugghe, B., and Lefebvre, V. (2001). The Transcription Factors L-Sox5 and Sox6 Are Essential for Cartilage Formation. Developmental cell 1, 277-290.

Solchaga, L.A., Gao, J., Dennis, J.E., Awadallah, A., Lundberg, M., Caplan, A.I., and Goldberg, V.M. (2002). Treatment of osteochondral defects with autologous bone marrow in a hyaluronan-based delivery vehicle. Tissue Eng. 8, 333-347. 
Solchaga, L.A., Johnstone, B., Yoo, J.U., Goldberg, V.M., and Caplan, A.I. (1999). High variability in rabbit bone marrow-derived mesenchymal cell preparations. Cell Transplant. 8, 511-519.

Solchaga, L.A., Temenoff, J.S., Gao, J., Mikos, A.G., Caplan, A.I., and Goldberg, V.M. (2005). Repair of osteochondral defects with hyaluronan- and polyester-based scaffolds. Osteoarthritis Cartilage 13, 297-309.

Solter, D. (2005). Politically correct human embryonic stem cells? N. Engl. J. Med. 353, 2321-2323.

Sorensen, A.L., Timoskainen, S., West, F.D., Vekterud, K., Boquest, A.C., Ahrlund-Richter, L., Stice, S.L., and Collas, P. (2010). Lineage-specific promoter DNA methylation patterns segregate adult progenitor cell types. Stem Cells Dev 19, 1257-1266.

St-Jacques, B., Hammerschmidt, M., and McMahon, A.P. (1999). Indian hedgehog signaling regulates proliferation and differentiation of chondrocytes and is essential for bone formation. Genes Dev. 13, 20722086.

Steadman, J.R., Rodkey, W.G., Briggs, K.K., and Rodrigo, J.J. (1998). The microfracture procedure: Rationale, technique, and clinical observations for treatment of articular cartilage defects. Sports, Traumatol Rel Res 20,61-70.

Steck, E., Fischer, J., Lorenz, H., Gotterbarm, T., Jung, M., and Richter, W. (2009). Mesenchymal stem cell differentiation in an experimental cartilage defect: restriction of hypertrophy to bone-close neocartilage. Stem Cells Dev 18, 969-978.

Steinwachs, M., and Kreuz, P.C. (2007). Autologous chondrocyte implantation in chondral defects of the knee with a type I/III collagen membrane: a prospective study with a 3-year follow-up. Arthroscopy 23, 381-387.

Stickens, D., Behonick, D.J., Ortega, N., Heyer, B., Hartenstein, B., Yu, Y., Fosang, A.J., Schorpp-Kistner, M., Angel, P., and Werb, Z. (2004). Altered endochondral bone development in matrix metalloproteinase 13-deficient mice. Development 131, 5883-5895.

Stockwell, R.A. (1971). The interrelationship of cell density and cartilage thickness in mammalian articular cartilage. J. Anat. 109, 411-421.

Sumiyoshi, K., Kubota, S., Ohgawara, T., Kawata, K., Abd El Kader, T., Nishida, T., Ikeda, N., Shimo, T., Yamashiro, T., and Takigawa, M. (2013). Novel role of miR-181a in cartilage metabolism. J. Cell.

Biochem. 114, 2094-2100

Takahashi, K., and Yamanaka, S. (2006). Induction of pluripotent stem cells from mouse embryonic and adult fibroblast cultures by defined factors. Cell 126, 663-676.

Tallheden, T., Brittberg, M., Peterson, L., and Lindahl, A. (2006). Human articular chondrocytes--plasticity and differentiation potential. Cells Tissues Organs 184, 55-67.

Tandogan, R.N., Taser, O., Kayaalp, A., Taskiran, E., Pinar, H., Alparslan, B., and Alturfan, A. (2004). Analysis of meniscal and chondral lesions accompanying anterior cruciate ligament tears: relationship with age, time from injury, and level of sport. Knee Surg. Sports Traumatol. Arthrosc. 12, 262-270.

Tang, Q.O., Carasco, C.F., Gamie, Z., Korres, N., Mantalaris, A., and Tsiridis, E. (2012). Preclinical and clinical data for the use of mesenchymal stem cells in articular cartilage tissue engineering. Expert Opin Biol Ther 12, 1361-1382.

Tatard, V.M., D'Ippolito, G., Diabira, S., Valeyev, A., Hackman, J., McCarthy, M., Bouckenooghe, T., Menei, P., Montero-Menei, C.N., and Schiller, P.C. (2007). Neurotrophin-directed differentiation of human adult marrow stromal cells to dopaminergic-like neurons. Bone 40, 360-373. 
Terada, N., Hamazaki, T., Oka, M., Hoki, M., Mastalerz, D.M., Nakano, Y., Meyer, E.M., Morel, L., Petersen, B.E., and Scott, E.W. (2002). Bone marrow cells adopt the phenotype of other cells by spontaneous cell fusion. Nature 416, 542-545.

Thai, T.H., Christiansen, P.A., and Tsokos, G.C. (2010). Is there a link between dysregulated miRNA expression and disease? Discov Med 10, 184-194.

Thomson, J.A., Itskovitz-Eldor, J., Shapiro, S.S., Waknitz, M.A., Swiergiel, J.J., Marshall, V.S., and Jones, J.M. (1998). Embryonic stem cell lines derived from human blastocysts. Science 282, 1145-1147.

Tognana, E., Borrione, A., De Luca, C., and Pavesio, A. (2007). Hyalograft C: hyaluronan-based scaffolds in tissue-engineered cartilage. Cells Tissues Organs 186, 97-103.

Toiyama, Y., Takahashi, M., Hur, K., Nagasaka, T., Tanaka, K., Inoue, Y., Kusunoki, M., Boland, C.R., and Goel, A. (2013). Serum miR-21 as a diagnostic and prognostic biomarker in colorectal cancer. J. Natl. Cancer Inst. 105, 849-859.

Tome, M., Lopez-Romero, P., Albo, C., Sepulveda, J.C., Fernandez-Gutierrez, B., Dopazo, A., Bernad, A., and Gonzalez, M.A. (2011). miR-335 orchestrates cell proliferation, migration and differentiation in human mesenchymal stem cells. Cell Death Differ. 18, 985-995.

Tonnesen, H.H., and Karlsen, J. (2002). Alginate in drug delivery systems. Drug Dev. Ind. Pharm. 28, 621630.

Treilleux, I., Mallein-Gerin, F., le Guellec, D., and Herbage, D. (1992). Localization of the expression of type I, II, III collagen, and aggrecan core protein genes in developing human articular cartilage. Matrix 12, 221-232.

Tuddenham, L., Wheeler, G., Ntounia-Fousara, S., Waters, J., Hajihosseini, M.K., Clark, I., and Dalmay, T. (2006). The cartilage specific microRNA-140 targets histone deacetylase 4 in mouse cells. FEBS Lett. 580, 4214-4217.

van Rooij, E., Purcell, A.L., and Levin, A.A. (2012). Developing microRNA therapeutics. Circ. Res. 110, 496-507.

Vandesompele, J., De Preter, K., Pattyn, F., Poppe, B., Van Roy, N., De Paepe, A., and Speleman, F. (2002). Accurate normalization of real-time quantitative RT-PCR data by geometric averaging of multiple internal control genes. Genome Biol 3, RESEARCH0034.

Vanlauwe, J., Almqvist, F., Bellemans, J., Huskin, J.P., Verdonk, R., and Victor, J. (2007). Repair of symptomatic cartilage lesions of the knee: the place of autologous chondrocyte implantation. Acta Orthop. Belg. 73, 145-158.

Vanlauwe, J., Saris, D.B., Victor, J., Almqvist, K.F., Bellemans, J., and Luyten, F.P. (2011). Five-year outcome of characterized chondrocyte implantation versus microfracture for symptomatic cartilage defects of the knee: early treatment matters. Am. J. Sports Med. 39, 2566-2574.

Vasiliadis, H.S., and Wasiak, J. (2010). Autologous chondrocyte implantation for full thickness articular cartilage defects of the knee. Cochrane Database Syst Rev, CD003323.

Vasudevan, S. (2012). Posttranscriptional upregulation by microRNAs. Wiley interdisciplinary reviews. RNA 3, 311-330.

Verzijl, N., DeGroot, J., Thorpe, S.R., Bank, R.A., Shaw, J.N., Lyons, T.J., Bijlsma, J.W., Lafeber, F.P., Baynes, J.W., and TeKoppele, J.M. (2000). Effect of collagen turnover on the accumulation of advanced glycation end products. J. Biol. Chem. 275, 39027-39031. 
Vista, E.S., and Lau, C.S. (2011). What about supplements for osteoarthritis? A critical and evidencedbased review. Int J Rheum Dis 14, 152-158.

Wagers, A.J., and Weissman, I.L. (2004). Plasticity of adult stem cells. Cell 116, 639-648.

Wagner, T.U. (2007). Bone morphogenetic protein signaling in stem cells--one signal, many consequences. The FEBS journal 274, 2968-2976.

Wagner, W., Wein, F., Seckinger, A., Frankhauser, M., Wirkner, U., Krause, U., Blake, J., Schwager, C., Eckstein, V., Ansorge, W., and Ho, A.D. (2005). Comparative characteristics of mesenchymal stem cells from human bone marrow, adipose tissue, and umbilical cord blood. Exp. Hematol. 33, 1402-1416.

Wakitani, S., Goto, T., Pineda, S.J., Young, R.G., Mansour, J.M., Caplan, A.I., and Goldberg, V.M. (1994). Mesenchymal cell-based repair of large, full-thickness defects of articular cartilage. J. Bone Joint Surg. Am. 76, 579-592.

Wakitani, S., Imoto, K., Yamamoto, T., Saito, M., Murata, N., and Yoneda, M. (2002). Human autologous culture expanded bone marrow mesenchymal cell transplantation for repair of cartilage defects in osteoarthritic knees. Osteoarthritis Cartilage 10, 199-206.

Wakitani, S., Mitsuoka, T., Nakamura, N., Toritsuka, Y., Nakamura, Y., and Horibe, S. (2004). Autologous bone marrow stromal cell transplantation for repair of full-thickness articular cartilage defects in human patellae: two case reports. Cell Transplant. 13, 595-600.

Walczak, B.E., McCulloch, P.C., Kang, R.W., Zelazny, A., Tedeschi, F., and Cole, B.J. (2008). Abnormal findings on knee magnetic resonance imaging in asymptomatic NBA players. J Knee Surg 21, 27-33.

Wang, L., Li, G., and Sugita, S. (2004). RalA-exocyst interaction mediates GTP-dependent exocytosis. J. Biol. Chem. 279, 19875-19881.

Watt, F.M., Lo Celso, C., and Silva-Vargas, V. (2006). Epidermal stem cells: an update. Curr. Opin. Genet. Dev. 16, 518-524.

Weiss, S., Hennig, T., Bock, R., Steck, E., and Richter, W. (2010). Impact of growth factors and PTHrP on early and late chondrogenic differentiation of human mesenchymal stem cells. J. Cell. Physiol. 223, 84-93.

Whittaker, J.P., Smith, G., Makwana, N., Roberts, S., Harrison, P.E., Laing, P., and Richardson, J.B. (2005). Early results of autologous chondrocyte implantation in the talus. J. Bone Joint Surg. Br. 87, 179-183.

Widuchowski, W., Widuchowski, J., Faltus, R., Lukasik, P., Kwiatkowski, G., Szyluk, K., and Koczy, B. (2011). Long-term clinical and radiological assessment of untreated severe cartilage damage in the knee: a natural history study. Scand. J. Med. Sci. Sports 21, 106-110.

Widuchowski, W., Widuchowski, J., and Trzaska, T. (2007). Articular cartilage defects: study of 25,124 knee arthroscopies. The Knee 14, 177-182.

Wilk, K.E., Briem, K., Reinold, M.M., Devine, K.M., Dugas, J., and Andrews, J.R. (2006). Rehabilitation of articular lesions in the athlete's knee. J. Orthop. Sports Phys. Ther. 36, 815-827.

Winter, A., Breit, S., Parsch, D., Benz, K., Steck, E., Hauner, H., Weber, R.M., Ewerbeck, V., and Richter, W. (2003). Cartilage-like gene expression in differentiated human stem cell spheroids: a comparison of bone marrow-derived and adipose tissue-derived stromal cells. Arthritis Rheum. 48, 418-429.

Winter, J., Jung, S., Keller, S., Gregory, R.I., and Diederichs, S. (2009). Many roads to maturity: microRNA biogenesis pathways and their regulation. Nature cell biology 11, 228-234. 
Wondrasch, B., Aroen, A., Rotterud, J.H., Hoysveen, T., Bolstad, K., and Risberg, M.A. (2013). The feasibility of a 3-month active rehabilitation program for patients with knee full-thickness articular cartilage lesions: the Oslo Cartilage Active Rehabilitation and Education Study. J. Orthop. Sports Phys. Ther. 43, 310-324.

Woods, A., Wang, G., and Beier, F. (2007). Regulation of chondrocyte differentiation by the actin cytoskeleton and adhesive interactions. J. Cell. Physiol. 213, 1-8.

Wu, L., Bluguermann, C., Kyupelyan, L., Latour, B., Gonzalez, S., Shah, S., Galic, Z., Ge, S., Zhu, Y., Petrigliano, F.A., Nsair, A., Miriuka, S.G., Li, X., Lyons, K.M., Crooks, G.M., McAllister, D.R., Van Handel, B., Adams, J.S., and Evseenko, D. (2013). Human developmental chondrogenesis as a basis for engineering chondrocytes from pluripotent stem cells. Stem cell reports 1, 575-589.

Xia, X., Zhang, Y., Zieth, C.R., and Zhang, S.C. (2007). Transgenes delivered by lentiviral vector are suppressed in human embryonic stem cells in a promoter-dependent manner. Stem Cells Dev 16, 167-176.

Yamashita, A., Nishikawa, S., and Rancourt, D.E. (2010). Identification of five developmental processes during chondrogenic differentiation of embryonic stem cells. PloS one 5, e10998.

Yamashita, S., Miyaki, S., Kato, Y., Yokoyama, S., Sato, T., Barrionuevo, F., Akiyama, H., Scherer, G., Takada, S., and Asahara, H. (2012). L-Sox 5 and Sox6 proteins enhance chondrogenic miR-140 microRNA expression by strengthening dimeric Sox9 activity. J. Biol. Chem. 287, 22206-22215.

Yamazaki, H., Tsuneto, M., Yoshino, M., Yamamura, K., and Hayashi, S. (2007). Potential of dental mesenchymal cells in developing teeth. Stem Cells 25, 78-87.

Yang, B., Guo, H., Zhang, Y., Chen, L., Ying, D., and Dong, S. (2011a). MicroRNA-145 regulates chondrogenic differentiation of mesenchymal stem cells by targeting Sox9. PloS one 6, e21679.

Yang, D., and Jones, K.S. (2009). Effect of alginate on innate immune activation of macrophages. Journal of biomedical materials research. Part A 90, 411-418.

Yang, J., Qin, S., Yi, C., Ma, G., Zhu, H., Zhou, W., Xiong, Y., Zhu, X., Wang, Y., He, L., and Guo, X. (2011b). MiR-140 is co-expressed with Wwp2-C transcript and activated by Sox9 to target Sp1 in maintaining the chondrocyte proliferation. FEBS Lett. 585, 2992-2997.

Yang, Y.C., Piek, E., Zavadil, J., Liang, D., Xie, D., Heyer, J., Pavlidis, P., Kucherlapati, R., Roberts, A.B., and Bottinger, E.P. (2003). Hierarchical model of gene regulation by transforming growth factor beta. Proc. Natl. Acad. Sci. U. S. A. 100, 10269-10274.

Yap, S.P., Xing, X., Kraus, P., Sivakamasundari, V., Chan, H.Y., and Lufkin, T. (2011). Generation of mice with a novel conditional null allele of the Sox9 gene. Biotechnology letters 33, 1551-1558.

Ying, Q.L., Nichols, J., Evans, E.P., and Smith, A.G. (2002). Changing potency by spontaneous fusion. Nature 416, 545-548.

Yoon, Y.S., Wecker, A., Heyd, L., Park, J.S., Tkebuchava, T., Kusano, K., Hanley, A., Scadova, H., Qin, G., Cha, D.H., Johnson, K.L., Aikawa, R., Asahara, T., and Losordo, D.W. (2005). Clonally expanded novel multipotent stem cells from human bone marrow regenerate myocardium after myocardial infarction. J. Clin. Invest. 115, 326-338.

Yoshida, C.A., and Komori, T. (2005). Role of Runx proteins in chondrogenesis. Crit. Rev. Eukaryot. Gene Expr. 15, 243-254. 
Ytrehus, B., Andreas Haga, H., Mellum, C.N., Mathisen, L., Carlson, C.S., Ekman, S., Teige, J., and Reinholt, F.P. (2004a). Experimental ischemia of porcine growth cartilage produces lesions of osteochondrosis. J. Orthop. Res. 22, 1201-1209.

Ytrehus, B., Carlson, C.S., Lundeheim, N., Mathisen, L., Reinholt, F.P., Teige, J., and Ekman, S. (2004b). Vascularisation and osteochondrosis of the epiphyseal growth cartilage of the distal femur in pigs-development with age, growth rate, weight and joint shape. Bone 34, 454-465.

Ytrehus, B., Ekman, S., Carlson, C.S., Teige, J., and Reinholt, F.P. (2004c). Focal changes in blood supply during normal epiphyseal growth are central in the pathogenesis of osteochondrosis in pigs. Bone 35, 12941306.

Yu, J., Vodyanik, M.A., Smuga-Otto, K., Antosiewicz-Bourget, J., Frane, J.L., Tian, S., Nie, J., Jonsdottir, G.A., Ruotti, V., Stewart, R., Slukvin, II, and Thomson, J.A. (2007). Induced pluripotent stem cell lines derived from human somatic cells. Science 318, 1917-1920.

Zhang, J., Tu, Q., Bonewald, L.F., He, X., Stein, G., Lian, J., and Chen, J. (2011). Effects of miR-335-5p in modulating osteogenic differentiation by specifically downregulating Wnt antagonist DKK1. J. Bone Miner. Res. 26, 1953-1963.

Zhao, Y., Glesne, D., and Huberman, E. (2003). A human peripheral blood monocyte-derived subset acts as pluripotent stem cells. Proc. Natl. Acad. Sci. U. S. A. 100, 2426-2431.

Zuk, P.A., Zhu, M., Mizuno, H., Huang, J., Futrell, J.W., Katz, A.J., Benhaim, P., Lorenz, H.P., and Hedrick, M.H. (2001). Multilineage cells from human adipose tissue: implications for cell-based therapies. Tissue Eng. 7, 211-228. 




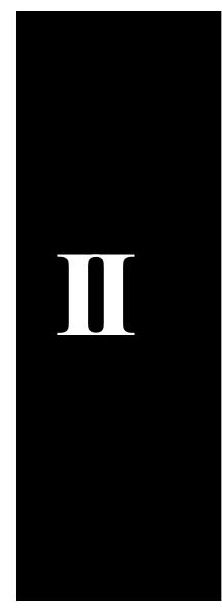





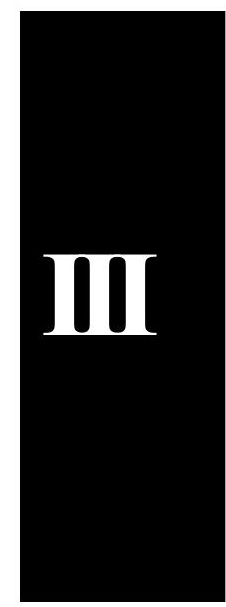





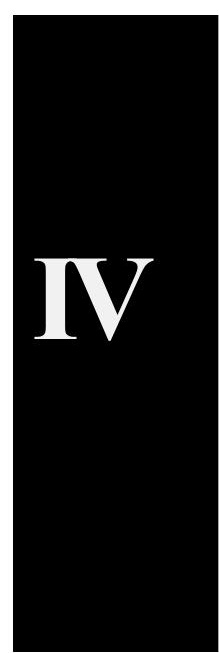





\section{Analysis of the effects of five factors relevant to in vitro chondrogenesis of human mesenchymal stem cells using factorial design and high throughput mRNA-profiling.}

Rune B. Jakobsen ${ }^{1,2}$, Esben Østrup ${ }^{1,3}$, Xiaolan Zhang ${ }^{4}$, Tarjei S. Mikkelsen ${ }^{4,5}$, Jan E. Brinchmann ${ }^{1,2,6^{*}}$.

${ }^{1}$ Norwegian Center for Stem Cell Research, Oslo University Hospital, Rikshospitalet, Oslo, Norway

${ }^{2}$ Department of Biochemistry, Institute of Basic Medical Sciences, The Medical Faculty, University of Oslo, Oslo, Norway

${ }^{3}$ Department of Biomaterials, Institute of Clinical Dentistry, University of Oslo, Norway

${ }^{4}$ Broad Institute, Cambridge, Massachusetts, USA

${ }^{5}$ Harvard Stem Cell Institute and Department of Stem Cell and Regenerative Biology, Harvard University, Cambridge, Massachusetts, USA

${ }^{6}$ Institute of Immunology, Oslo University Hospital, Rikshospitalet, Oslo, Norway 
Contact info:

Rune Bruhn Jakobsen, Telephone: +47 92092973, Fax: +47 22851058,

Email: r.b.jakobsen@medisin.uio.no

Esben Østrup, Telephone: +47 94219943, Fax: +47 22851058

Email: esben.ostrup@odont.uio.no

Xiaolan Zhang, Telephone: +1 617-714-7813, Fax: + 1 617-714-8102

Email: xzhang@broadinstitute.org

Tarjei S. Mikkelsen, Telephone: + 1 617-714-7772, Fax: + 1 617-714-8102,

Email: tarjei@broadinstitute.org

\section{*CORRESPONDING AUTHOR:}

Jan E. Brinchmann, Telephone: +47 22840489, Fax: +47 22851058,

E-mail: jan.brinchmann@rr-research.no,

Postal address: Norwegian Center for Stem Cell Research, Pb. 1112 Blindern, N-0317

Oslo, Norway. 


\begin{abstract}
The in vitro process of chondrogenic differentiation of mesenchymal stem cells for tissue engineering has been shown to require three-dimensional culture along with the addition of differentiation factors to the culture medium. In general, this leads to a phenotype lacking some of the cardinal features of native articular chondrocytes and their extracellular matrix.

The factors used vary, but regularly include members of the transforming growth factor $\beta$ superfamily and dexamethasone, sometimes in conjunction with fibroblast growth factor 2 and insulin-like growth factor 1 , however the use of soluble factors to induce chondrogenesis has largely been studied on a single factor basis. In the present study we combined a factorial quality-by-design experiment with highthroughput mRNA profiling of a customized chondrogenesis related gene set as a tool to study in vitro chondrogenesis of bone marrow derived mesenchymal stem cells in alginate. 48 different conditions of transforming growth factor $\beta 1,2$ and 3, bone morphogenetic protein 2, 4 and 6, dexamethasone, insulin-like growth factor 1, fibroblast growth factor 2 and cell seeding density were included in the experiment.

The analysis revealed that the best of the tested differentiation cocktails included transforming growth factor $\beta 1$ and dexamethasone. Dexamethasone acted in synergy with transforming growth factor $\beta 1$ by increasing many chondrogenic markers while directly downregulating expression of the pro-osteogenic gene osteocalcin. However, all factors beneficial to the expression of desirable hyaline cartilage markers also induced undesirable molecules, indicating that perfect chondrogenic differentiation is not achievable with the current differentiation protocols.
\end{abstract}




\section{Introduction}

Mesenchymal stem cells (MSCs) have been advocated as a useful cell source for tissue engineering. MSCs were originally isolated from bone marrow, but has later been found in and isolated from numerous tissues[1,2]. They can be readily expanded in vitro and differentiated into tissues of mesodermal and, in some instances, ectodermal lineages[3,4]. Clinically MSCs have shown promising potential in treatments of graft-versus-hostdisease and in repair of full-thickness cartilage defects[5,6].

The in vitro process of directed differentiation of mesenchymal stem cells has been widely studied. Chondrogenic differentiation of MSCs has been shown to require the use of either high-density cell pellet, micro-mass cultures or a scaffold allowing for threedimensional culture[7-9] along with the addition of differentiation factors to the culture medium[10-13].

The differentiation factors have traditionally included factors from the TGF superfamily such as transforming growth factor $\beta$ (TGF $\beta)[7,14,15]$ and/or bone morphogenetic protein (BMP) $[9,16,17]$ along with the steroid hormone dexamethasone (DEX). Other factors used are fibroblast growth factor 2 (FGF2)[15,18,19] and insulin-like growth factor 1 (IGF1)[20,21]. Traditionally, the use of soluble factors to induce chondrogenesis has largely been studied on a single factor basis or with simple combinations of a few factors. However, optimizing differentiation conditions one factor at a time is time consuming, and does not take into account interdependency between factors, which is likely to play a role in growth factor mediated differentiation. Factorial analysis is commonly used in industrial processes as a statistically and scientifically sound way of analyzing interplay between several factors on a predefined outcome. Factorial design 
(often termed quality-by-design) has been used for optimization of protocols in a variety of industries and research areas including pharmaceutical studies and manufacturing, stem cell biology, polymer production and tissue engineering[22-24].

Previously, expression profiling of medium to large sets of genes on multiple samples has been done using microarray hybridization technology with a relative high cost per individual sample. Smaller sets of genes have often been investigated using quantitative polymerase chain reactions (qPCR), though upscaling of qPCR experiments rapidly exceeds practically and economically feasible numbers of reactions. However, the introduction of digital and highly multiplexed mRNA-profiling (Nanostring nCounter) has made it possible and cost-effective to analyze large number of samples on predefined gene sets of up to 800 genes with an accuracy equal to single-plex qPCR[25]. This may be performed directly on cell lysates, thus bypassing the variability introduced by RNA isolation and conversion to cDNA which is necessary in microarrays and RT-qPCR[26].

In the present study we undertook a detailed comparison of all possible combinations of five commonly used differentiation factors in a fully humanized culture system: TGF $\beta 1$, BMP2, dexamethasone, FGF2 and IGF1 used for in vitro chondrogenesis of MSCs established in 3D culture in alginate hydrogels, including a comparison of the three isoforms of the TFG $\beta$ growth factor (TGF $\beta 1$, TGF $\beta 2$ and TGF $\beta 3$ ) and three of the isoforms of BMP (BMP2, BMP4 and BMP6). Our aim was to explore factorial design and digital mRNA profiling as tools to characterize directed differentiation of MSCs and to validate the most commonly used chondrogenic growth factors. 


\section{Methods and materials}

All chemicals were purchased from Sigma-Aldrich unless otherwise stated.

\section{Ethics statement}

The harvest of bone marrow from voluntary donors was approved by the Regional Committee for Medical Research Ethics, Southern Norway. Informed consent was obtained from all donors before the harvest procedure.

\section{Cell harvest and culture}

Bone marrow aspirates were obtained from the iliac crest of three healthy donors as previously described[27]. The isolation and culture procedure is given in the Supporting Information Methods and materials.

\section{Medium and supplements}

Growth medium for monolayer cultures contained $2 \mathrm{U} / \mathrm{mL}$ heparin, $100 \mathrm{U} / \mathrm{mL}$ penicillin, $100 \mu \mathrm{g} / \mathrm{mL}$ streptomycin and 2,5 $\mu \mathrm{g} / \mathrm{mL}$ amphotericin B in DMEM F-12, with $20 \%$ human platelet lysate (hPL) (Supporting Information Methods and materials) added for the first passage and $10 \%$ for all subsequent passages.

Basic chondrogenic differentiation medium (bCDM) contained $1 \mathrm{mM}$ sodium pyruvate, $0.1 \mathrm{mM}$ ascorbic-acid-2-phosphate, $1 \%$ ITS (25 mg insulin, $25 \mathrm{mg}$ transferrin and $25 \mu \mathrm{g}$ sodium selenite) and 1,25 mg/mL human serum albumin in high-glucose DMEM-F12 (4,5 g/L). bCDM was supplemented with 500 ng/mL BMP2 (InductOs; Wyeth, Taplow, UK), BMP4 or BMP6 (R\&D Systems, Minneapolis, MN), 10 ng/mL TGF $\beta 1$, TGF $\beta 2$ or 
TGFß3 (R\&D Systems), 100 nM dexamethasone, 10 ng/mL FGF2 (R\&D Systems) and/or 100 ng/mL IGF1.

\section{Validation of cells as MSC}

Cells used for experiments in passage 2 or 3 were validated as MSCs by flow cytometry and differentiation assays as described in Supporting Information Methods and materials.

\section{$3 D$ cell culture}

Cells in passage 2 or 3 were trypsinized, counted, washed in PBS and seeded into a selfgelling alginate scaffold (NovaMatrix, Sandvika, Norway) as described previously and in Supporting Information Methods and materials[28].

\section{Experimental design}

We investigated a total of 48 different conditions (Figure 1A). Five factors: TGF $\beta 1$, IGF1, DEX, FGF2 and BMP2 were investigated in two-level (present or not) full $2^{5}$ factorial design experiments. Concentrations were based on typical use in the literature[7,1517,21]. This gave a total of 32 conditions in each experiment, which was repeated with cells from three donors at two time points each: days 1 and 7 . In addition a modified design investigating TGF $\beta$ isoforms 1, 2 and 3 and BMP isoforms 2, 4 and 6 was also performed, including an experiments where the cell density was varied between $1.25 \times 10^{6}$ and $2 \times 10^{7}$ cells $/ \mathrm{mL}$ at $\log (2)$-intervals. Design of the experiments was done with MiniTAB 16 (Minitab Inc, State College, PA). At the end of the experiments discs were divided in halves, snap-frozen in liquid nitrogen and stored at $-80^{\circ} \mathrm{C}$. Negative control disc cultures were performed in the same way using bCDM only, while positive controls were discs supplemented with our to date standard chondrogenic differentiation cocktail 
consisting of TGF $\beta 1$, BMP2 and DEX. Positive control samples were collected at day 7 , 14 and 21 with additions of extra discs fixed in 4\% paraformaldehyde for immunohistochemistry.

\section{Digital $m R N A$ profiling and quantitative polymerase chain reaction}

Frozen discs were crushed in liquid nitrogen with a pestle (Argos Technologies, Elgin, IL), lysed in RLT buffer and homogenized (QiaShredder, Qiagen, Venlo, Netherlands). Samples were then either directly used for digital mRNA profiling with the Nanostring nCounter technology[25] (NanoString Technologies, Seattle, WA) or RNA was extracted (RNeasy Mini kit, Qiagen). For qPCR, after DNase I treatment (Ambion; Life Technologies, Carlsbad, CA), reverse transcription (RT) was performed according to protocol (High-Capacity cDNA archive Kit; Applied Biosystems, Foster City, CA) using 200 ng total RNA per RT reaction and analyzed with the primers for peroxisome proliferator-activated receptor gamma and osteomodulin (PPARG Hs01115513_m1, OMD Hs00192325_m1, Applied Biosystems).

For the digital mRNA profiling, a custom chondrogenic gene set consisting of 364 genes (Table S1) including endogenous controls was established on the background of genes known or suspected to be affected by chondroskeletogenesis[28]. For analysis of lysate vs. purified mRNA performance, the pre-designed NanoString 48-plex Customer Assay Evaluation (CAE) kit was used instead. Sample preparation and hybridization was performed according to the manufacturer's instructions with either $100 \mathrm{ng}$ of total RNA or lysate equivalent to 10.000 cells. All hybridizations were incubated at $65^{\circ} \mathrm{C}$ purified and counted on the nCounter Prep Station and Digital Analyzer (NanoString Technologies). 


\section{Data analysis and statistics}

Normalization for lane-to-lane variation and positive spike-in-control series were performed according to the manufactures protocol[29] using Microsoft Excel (Microsoft , Redmond, WA). The geometric mean of the five best endogenous control genes identified by NormFinder was used to normalize the data[30]. Further data normalization was performed in the R statistical application (http://www.R-project.org/) including log transformation using the "vsn" package[31]. MiniTAB was used to fit a statistical regression model to analyze main effects, two and three factor interactions with significance assumed for $\mathrm{p}$-values less than 0.05 in a multivariate analysis of variance on the normalized data. If needed, transformation of the responses was used to make the residuals exhibit normality as judged by normality plots. Pearson's correlation between expression values in lysates and RNA and Spearman's correlation between rankings of conditions day 1 and day 7 were calculated with Prism 6 (Graphpad, San Diego, CA).

To make graphical representations of wanted and unwanted genes the data were Studentized subtracting the mean expression of each gene across all conditions divided by the standard deviation. For analysis of significantly changed genes and gene set enrichment analysis the data were analyzed in $\mathrm{R}$ using the "Limma" package[32] to fit a linear model to the data. Cut-off values were set to twofold difference in expression values with a false discovery rate of $5 \%($ FDR $<0.05)$. The "ade4"[33] package in R was used to perform a two-dimensional principal component analysis on the normalized data. 


\section{Results}

\section{Characterization of cells and validation of the use of lysates for mRNA profiling}

Surface antigen profiles were obtained of the expanded cells at passage 2 (Figure S1A). Cells readily differentiated into adipogenic and osteogenic lineages verified by extensive staining of lipid droplets and calcium deposits and upregulation of $P P A R G$ and $O M D$ (Figure S1B and C). Cells also showed differentiation into the chondrogenic lineage with upregulation of gene expression and synthesis of proteins representing key chondrogenic markers (Figure S1D and E). To evaluate if lysate of cells in alginate discs could be used instead of RNA, lysate and RNA isolated from matching samples at three timepoints grown under standard chondrogenic conditions were analyzed. Results showed highly significant correlations $(\mathrm{p}<0.0001)$ for all pairs with coefficients of determination $\left(\mathrm{R}^{2}\right)$ ranging from 0.92 to 0.97 (Figure S2). This validated the use of lysate through the rest of the study.

\section{Principal component analysis on the full gene set}

Principal component analysis (PCA) is a powerful way of reducing the dimensionality of a large data set in an unbiased way to identify clustering behaviour[34]. To see if the mRNA profiling of the full chondrogenic gene set reflected the studied conditions both regarding factors and temporal spatialization, we performed a PCA on the full dataset in all conditions at all timepoints (Figure 1B). This revealed that day 1 and day 7 samples clustered together, with larger differences observed within the day 7 cluster. We next limited the the PCA to only day 0 (untreated cells) and the full $2^{5}$-factorial design at day 1 (Figure 1C) or day 7 (Figure 1D) to allow for a more detailed analysis of the individual factors and combinations. At both timepoints it was readily apparent that conditions 
clustered according to TGF $\beta 1$ exposure, with unexposed conditions being closer to undifferentiated MSCs. On day 7 (Figure 1D) it was also evident that adding IGF1 led to only very minor differences (see for example conditions 25 and 27, 2 and 4 or 9 and 11). Notably, the 6 conditions found in the lower right quadrant of the plot all included TGF $\beta 1$ and DEX.

Interactions between TGF $1, D E X, B M P 2, I G F 1$ and $F G F 2$ evaluated by changes in selected gene subsets

The full custom-made chondrogenic gene set comprised 364 genes including endogenous reference genes. It included both genes that are hallmarks of hyaline cartilage, but also genes that mark other differentiation processes such as adipogenesis or osteogenesis. To study the effects of the individual factors specifically on chondrogenesis we prespecified two subsets of genes: a "wanted" marker group comprised of genes coding for extracellular matrix (ECM) molecules known to be hallmarks of native hyaline cartilage[35], and the negative "unwanted" marker group comprised of genes coding for extracellular molecules distinctive for other cartilage types, but also genes coding for major transcription factors of other lineages such as adipose tissue or bone (Table 1). The mean expression of "wanted" or "unwanted" markers was used as responses when fitting a statistical regression model to the full factorial design. This allowed us to study the main effects of individual factors and significant interactions between factors on chondrogenesis. The normal plots of standardized effects using wanted and unwanted markers on day 1 (Figure S3A and D) and day 7 (Figure 2A and D) show the factors and interactions that significantly affected the wanted and unwanted responses. Focusing on day 7, TGF $\beta 1$, DEX and BMP2 affected the wanted markers significantly in the desired 
direction and FGF2 in the opposite direction (Figure 2B). A more complete description is seen when analyzing the significant two-way interactions of TGF $\beta 1$ with DEX and TGF $\beta 1$ with BMP2 (Figure 2C). The effect on wanted markers of TGF $\beta 1$ was dependent on the presence of DEX. TGF $\beta 1$ on its own had a much smaller effect than when added in the presence of DEX. For the interaction of TGF $\beta 1$ with BMP2 the opposite was true: adding TGF $\beta 1$ in the presence of BMP2 led to a smaller absolute increase in wanted marker expression than when TGF $\beta 1$ was added alone (Figure 2D). Only one three-way interaction, that of TGF $\beta 1$, DEX and BMP2, was found to significantly affect wanted markers. However, the standardized effect was small, and showed that both the TGF $\beta 1 / B M P 2$ and the TGF $\beta 1 / D E X$ interaction was affected by the addition of the third factor, which in both cases decreased the total effect slightly (Table S2). The effects of the differentiation factors on the expression of the unwanted marker genes were very similar to that seen for the wanted genes, with a few notable differences (Figure 2E, F, G and H). First, DEX alone did not have a significant effect (Figure 2F, Table S2). Second, both TGF $\beta 1$ and BMP2 alone increased unwanted marker expression, but in combination TGF $\beta 1$ or BMP2 did not increase unwanted expression above that seen for each of them alone (Figure 2G). FGF2 seemed to reduce the expression of wanted genes considerably, while IGF1 did not impact on this gene set at all (Figure 2B and F)

\section{Identifying optimal differentiation conditions from the expression of wanted and} unwanted genes

As the PCA was done on the full gene set, we next wanted to explore if the changes in gene expression that segregated the different conditions reflected a desired change in terms of the expression of the wanted or unwanted gene subsets (Figure 3A). 
Interestingly, we found a highly significant $(\mathrm{P}<0.0001)$ correlation between the ranking of conditions on day 1 and day 7 (Figure 3B), which shows that the changes in mRNA expression that arise soon after induction of differentiation can predict the direction of later changes. Corroborating the PCA, plots of the summary score of wanted and unwanted markers show larger separation in the day 7 samples (Figure 3B). In the detailed view of day 0 and the full $2^{5}$-factorial design on day 7 (Figure $3 \mathrm{C}$ ) it is clear that conditions 9 and 11 are the most favorable, with low scores for unwanted markers and the highest scores of wanted markers overall. It is also apparent from the color coding that TGF $\beta 1$ is substantially affecting expression of wanted markers in the desired direction, yet also increasing the expression of unwanted markers. DEX, on the other hand, seems to increase only expression of wanted markers if added in the presence of TGF $\beta 1$. To support these findings we performed a gene set enrichment analysis for the wanted and unwanted gene sets. Conditions significantly enriched for the wanted gene set (FDR $<0.05$ ), but not significantly enriched for the unwanted gene set, are highlighted in bold sorted by the FDR (Table 2). The top ranked conditions match the previous findings and a heatmap of the expression of wanted and unwanted markers of the top ten conditions (Figure 4) further visually confirms the changes with lower expressions of unwanted markers and higher expression of wanted markers in conditions 9, 11, 10 and 12. However, from the heatmap it is also apparent that individual unwanted and wanted genes such as $L U M, A L P L, C O L 10 A 1$ and PPARG did not change in the desired direction. The three TGF $\beta$ isoforms tested did not show substantial differences in the mean expression of wanted and unwanted markers. Figure S4A shows that addition of any TGF $\beta$ isoform increased both wanted and unwanted markers, addition of DEX to any of 
these increased wanted markers further and addition of BMP 2 in the presence of TGF $\beta$ and DEX decreased unwanted marker expression slightly. In the comparison of BMP isoforms we found that addition of any of the three BMP isoforms alone increased both wanted and unwanted marker expression, and addition of TGF $\beta 1$ further increased the expression of wanted markers (Figure S4B). Cell density upon induction of chondrogenesis affected expression both on day 1 and day 7 (Figure S4C). The expression of wanted markers on day 7 increased as the cell density was increased from $1.25 \times 10^{6}$ to $10 \times 10^{6}$ cells $/ \mathrm{mL}$. However, further increasing the cell density to $2 \times 10^{7}$ cells/mL reduced the expression of wanted genes substantially.

Genes uniformly affected by single factors across all conditions

To elucidate effects of individual factors on genes other than the selected wanted or unwanted marker genes we performed an analysis of differentially expressed genes between the bCDM and all other conditions (Figure S5). Figure 5 shows the genes that were consistently up or down regulated in all conditions with any one of the five factors. It is evident that the expression for several genes is completely dependent on the presence of a specific factor. The pro-osteoblastic gene $B G L A P$, for example, which codes for osteocalcin, was downregulated in all conditions containing DEX, but not affected in any other conditions. The matrix metallopeptidase $M M P 1$, which specifically degrades type I, II and III collagen, was also almost exclusively downregulated in conditions containing DEX. Genes consistently upregulated by DEX included $M M P 7$, previously shown to correlate with chondrocyte maturation[36] and the tissue inhibitor of metalloproteinases 4 (TIMP4), known to be upregulated in response to cartilage injury and degradation[37]. Another example worth special attention is the WNT-signalling modulator SFRP4, 
known to be upregulated during adipogenesis[38], shown here to be consistently downregulated by DEX, and upregulated in conditions with BMP2 and no DEX, except where BMP2 was added alone or with IGF1 only. COL10A1, a known marker of hypertrophy[39] was consistently and exclusively upregulated in conditions with TGF $\beta 1$. Unlike practically all the other molecules upregulated by TGF $\beta 1$, for COL10A1 the absence of TGF $\beta 1$ could not be compensated for by the addition of BMP2. COL2A1, which encodes for the major collagen of hyaline collage, is somewhat surprisingly not upregulated consistently by any one factor, though it is consistently upregulated in all conditions containing TGF $\beta 1$ and DEX (Figure S5). Interestingly, FGF2 could be seen to inhibit the upregulatory effect of TGF $\beta 1$ or BMP2 on COL2A1 in all conditions where DEX was not also added. Further substantiating that DEX plays an important role in chondrogenesis is that $P R G 4$, encoding the surface lubricant lubricin [40], was only upregulated in conditions with DEX without TGF $\beta$-superfamily ligands (Figure S5).

\section{Genes differentially regulated between key conditions}

Finally, we examined genes differentially expressed between key conditions. In particular, we focused on the effect of adding DEX to either TGF $\beta 1$ or to TGF $\beta 1+B M P 2$, or adding BMP2 to either TGF $\beta 1$ or TGF $\beta 1+$ DEX (Figure 6A and B and Figure S6). Adding DEX to TGF $\beta 1$ changed 115 genes significantly, and adding DEX to TGF $\beta 1$ with BMP2 changed 110 genes, with an overlap of 77 genes (Figure S7 and Table S3). Several desired genes were upregulated by DEX such as $A C A N, C O L 2 A 1$ and $S O X 9$ while undesired genes such as the collagen degrading metallopeptidase $M M P 13$ and the osteogenic transcription factor $R U N X 2$ were downregulated by DEX. 
As expected, adding BMP2 as the second TGF $\beta$-superfamily signalling molecule to either TGF $\beta 1$ alone or TGF $\beta 1$ with DEX changed only 17 and 14 genes respectively Surprisingly, only the upregulated gene SOCS2 was common between these gene sets, showing that DEX importantly affects the way TGF $\beta 1$ stimulated MSCs respond to BMP2.

Given that the beneficial effect of DEX has been amply proven, and IGF1 and FGF2 have been shown to not have effects or even predominantly negative effects, the remaining question was whether BMP2 should be added to the combination of TGF $\beta 1$ and DEX. The answer to this question, at the single gene expression level, is found in Figure 6D. The most highly upregulated gene, $N O G$, encodes a polypeptide noggin that binds and inactivates BMPs belonging to the TGF $\beta$-superfamily, particularly BMP4[41,42]. Also the addition of BMP2 on a background of TGF $\beta 1+$ DEX leads to downregulation of BMP4 at the mRNA level, which could be a direct effect of BMP2 or perhaps an effect by noggin also on BMP4 mRNA expression. NOTCH1, which has been shown to be required in early chondrogenesis but must be turned off for full chondrogenesis to occur[43,44], was also downregulated. In addition, several other downregulated genes such as $D P T, F G F R 1$ and $T G F B 1$ are likely to have pro-chondrogenic effects $[15,45]$. One positive effect of BMP2 was the downregulation of COL3A1, a collagen frequently coexpressed with type I collagen in connective tissues[46]. In total, these data indicate that addition of BMP2 to a chondrogenic cocktail already consisting of TGF $\beta 1$ and DEX will not improve cartilage formation, at least judging by the expression of genes of relevance for chondrogenesis. 


\section{Discussion}

Directed differentiation of stem cells into chondrocytes in vitro has been shown to require both three-dimensional culture and environmental ques in the form of growth factors[7,16,17,47]. These cocktails of growth factors have largely been studied by manipulating one factor at a time, which is laborous and time consuming. We show here that high-throughput gene profiling makes it feasible to perform larger scale experiments with statistical design of experiments, allowing for sound conclusions on the involvement of many simultaneously investigated factors[24,48,49].

In the present study we used this approach to dissect the expression of a chondrogenesis relevant gene set during in vitro chondrogenesis of MSCs subjected to 48 different conditions of growth factors and cell densities. We found that only three of the factors (TGF $\beta 1$, DEX, and BMP2) directly increased the expression of chondrogenic markers significantly. Adding FGF2 or IGF1, either alone or in combination with other factors, had either no effect or predominantly negative effects on the expression of chondrogenic genes.

TGF $\beta 1$ is the most extensively used factor for inducing chondrogenesis in directed differentiation of MSCs[10]. The present data show that the related factors TGF $\beta 2$ and 3, but no single other factor studied here, can replace its positive effects on chondrogenic differentiation of human MSCs. Next, we found that adding DEX to TGF $\beta 1$ changed more that 100 of the investigated genes significantly, with the vast majority of changes being favourable for chondrogenesis. This is in line with the use of DEX in most of the published literature[7,10,14], although a recent publication actually concluded that DEX should be omitted [50]. The present study extends current knowledge by describing 
which of a large set of relevant genes are changed by each of these factors, and then by two together.

BMPs can, like the TGF $\beta$ isoforms, promote MSC differentiation into chondrocytes $[51,52]$. However, BMPs and TGF $\beta$ have also been described to exhibit antagonistic activities in many tissues[53]. We found that both factors increased wanted chondrogenic markers on their own. Interestingly, the two combinations identified with the best ratio of wanted to unwanted genes and highest mean expression of wanted markers contained both TGF $\beta 1$ and BMP2. However the effect of adding either factor together with the other was marginal and not synergistical on either wanted or unwanted markers. This is most likely explained by the molecular mechanism for the actions of these factors. Both BMP2 and TGF $\beta 1$ are ligands of the transformings growth factor $\beta$ superfamily and act by binding to specific type II receptors, which recruits the corresponding type I receptor, ultimately leading to phosporylation of receptor-SMADs. Even though BMP2 works mainly through SMAD1, 5 and 8 and TGF $\beta 1$ through SMAD2 and 3, there are known interactions between the two systems such as the competitive occupation of the common downstream effector SMAD4[53]. Also it seems that adding BMP2 to TGF $\beta 1$ and DEX does not lead to a significant upregulation of any genes positively related to chondrogenesis, but rather to an endogenous modulation of BMP4. Taken together, there may be more reasons to exclude BMP2 than to include it in a chondrogenic differentiation cocktail, although it could have a role in a system with sequential cocktails for different parts of chondrogenesis, as illustrated by the effect on PRG4. The FGF2 treatment did not lead to an increase in the chondrogenic gene expression. On the contrary, a significant reduction was seen in the general gene expression of both 
wanted and unwanted genes when FGF2 was added to the basic differentiation cocktail. In line with our finding, it has been published that FGF2 may abolish chondrogenesis when combined with TGF $\beta 1$ and BMP-6[54]. On the other hand, FGF2 has recently been described to enhance the potential of MSCs for use in tissue engineering of cartilage $[18,55]$ when used as a mitogen in the expansion phase prior to the differentiation.

We found that IGF1 did not change the general expression of either wanted or unwanted genes significantly, which is contrary to some previous publications[21]. IGF1 has been shown to be expressed in articular cartilage and regulate proteoglycan metabolism[56] and it has a distinct expression profile during embryogenic chondrogenesis[57]. However our finding is in line with other publications failing to find effects of using IGF1 to induce chondrogenesis[58,59].

There are limitations to our approach. We only considered gene expression on the level of transcribed mRNA, which does not necessarily correlate with protein synthesis[60]. We also utilized a two-level factorial design with either absence or presence of the investigated growth factors, which did not allow an assesment of the role that different factor concentrations might play. Considering these limitations, we propose that the method presented here could be adapted to screen large numbers of molecules that could enhance chondrogenesis. We also believe that the method described could be valuably expanded to testing several concentrations of factors, which would also allow a statistical analysis with response optimization to be performed[24]. Further supporting the feasibility of our approach in larger screening experiments, is our finding that gene profiling can be performed directly on lysates without any loss in assay quality. Also the 
finding that changes in gene expression seen just one day after induction predicts later changes, potentially allows for a simpler design with just one time point, likely earlier than the one week mark chosen in the present study. Combined, the implications of these findings could decrease both cost and workload considerably in future experiments. Finally, larger screening experiments could be efficiently performed in a fractionalized factorial design allowing for sound conclusions without increasing the number of experiments[23,61], for example in order to test temporal spatialization of chondrogenic factors to more exactly mirror the conditions known from embryogenesis of cartilage.

\section{Conclusion}

In this study we have shown that high-throughput mRNA profiling can be efficiently performed on lysates of MSCs during in vitro chondrogenesis in alginate. A thorough analysis revealed that the cocktail of growth factors leading to the most efficient upregulation of wanted chondrogenic markers was a combination of TGF $\beta$ and DEX. Adding BMP2 lead to a slightly higher mean expression of wanted markers but did not significantly upregulate key positive genes and led to a downregulation of endogenous BMP4 and TGF $\beta 1$ expression, and may therefore be expendable. DEX, on the other hand, worked synergistically with TGFB1 in increasing wanted marker expression and was also directly downregulating expression of the unwanted marker BGLAP. All factors beneficial to the expression of wanted hyaline cartilage markers also introduced an induction of unwanted markers, with the exception of DEX alone. Upregulation of COL10A1 was seen in all conditions containing TGF $\beta 1$ indicating that perfect 
differentiation to hyaline cartilage is not achievable with the current differentiation protocols.

\section{Acknowledgement}

We acknowledge the help from Axel Küchler Ph.D. in assembling the custom

chondrogenic gene set, and Karen Johanne Beckstrøm, MSc for preparing hPL. 


\section{REFERENCES}

1. Friedenstein AJ, Chailakhjan RK, Lalykina KS (1970) The development of fibroblast colonies in monolayer cultures of guinea-pig bone marrow and spleen cells. Cell Tissue Kinet 3: 393-403.

2. Steinert AF, Rackwitz L, Gilbert F, Noth U, Tuan RS (2012) Concise review: the clinical application of mesenchymal stem cells for musculoskeletal regeneration: current status and perspectives. Stem Cells Transl Med 1: 237-247.

3. Kuroda Y, Kitada M, Wakao S, Nishikawa K, Tanimura Y, et al. (2010) Unique multipotent cells in adult human mesenchymal cell populations. Proc Natl Acad Sci U S A 107: 8639-8643.

4. Boulland JL, Mastrangelopoulou M, Boquest AC, Jakobsen R, Noer A, et al. (2013) Epigenetic regulation of nestin expression during neurogenic differentiation of adipose tissue stem cells. Stem Cells Dev 22: 1042-1052.

5. Le Blanc K, Rasmusson I, Sundberg B, Gotherstrom C, Hassan M, et al. (2004) Treatment of severe acute graft-versus-host disease with third party haploidentical mesenchymal stem cells. Lancet 363: 1439-1441.

6. Wakitani S, Mitsuoka T, Nakamura N, Toritsuka Y, Nakamura Y, et al. (2004) Autologous bone marrow stromal cell transplantation for repair of full-thickness articular cartilage defects in human patellae: two case reports. Cell Transplant 13: 595-600.

7. Johnstone B, Hering TM, Caplan AI, Goldberg VM, Yoo JU (1998) In vitro chondrogenesis of bone marrow-derived mesenchymal progenitor cells. Exp Cell Res 238: 265-272.

8. Holtzer H, Abbott J, Lash J, Holtzer S (1960) The Loss of Phenotypic Traits by Differentiated Cells in Vitro, I. Dedifferentiation of Cartilage Cells. Proc Natl Acad Sci U S A 46: 1533-1542.

9. Mackay AM, Beck SC, Murphy JM, Barry FP, Chichester CO, et al. (1998) Chondrogenic differentiation of cultured human mesenchymal stem cells from marrow. Tissue Eng 4: 415-428. 
10. Puetzer JL, Petitte JN, Loboa EG (2010) Comparative review of growth factors for induction of three-dimensional in vitro chondrogenesis in human mesenchymal stem cells isolated from bone marrow and adipose tissue. Tissue Eng Part B Rev 16: 435-444.

11. Barrilleaux B, Phinney DG, Prockop DJ, O'Connor KC (2006) Review: ex vivo engineering of living tissues with adult stem cells. Tissue Eng 12: 3007-3019.

12. Benders KE, van Weeren PR, Badylak SF, Saris DB, Dhert WJ, et al. (2013) Extracellular matrix scaffolds for cartilage and bone regeneration. Trends Biotechnol 31: 169-176.

13. Tuan RS (2013) Regenerative medicine in 2012: the coming of age of musculoskeletal tissue engineering. Nat Rev Rheumatol 9: 74-76.

14. Yoo JU, Barthel TS, Nishimura K, Solchaga L, Caplan AI, et al. (1998) The chondrogenic potential of human bone-marrow-derived mesenchymal progenitor cells. J Bone Joint Surg Am 80: 1745-1757.

15. Frenz DA, Liu W, Williams JD, Hatcher V, Galinovic-Schwartz V, et al. (1994) Induction of chondrogenesis: requirement for synergistic interaction of basic fibroblast growth factor and transforming growth factor-beta. Development 120: 415-424.

16. Sekiya I, Colter DC, Prockop DJ (2001) BMP-6 enhances chondrogenesis in a subpopulation of human marrow stromal cells. Biochem Biophys Res Commun 284: 411-418.

17. Sekiya I, Larson BL, Vuoristo JT, Reger RL, Prockop DJ (2005) Comparison of effect of BMP-2, -4 , and -6 on in vitro cartilage formation of human adult stem cells from bone marrow stroma. Cell Tissue Res 320: 269-276.

18. Handorf AM, Li WJ (2011) Fibroblast growth factor-2 primes human mesenchymal stem cells for enhanced chondrogenesis. PLoS One 6: e22887.

19. Solchaga LA, Penick K, Goldberg VM, Caplan AI, Welter JF (2010) Fibroblast growth factor- 2 enhances proliferation and delays loss of chondrogenic potential in human adult bone-marrow-derived mesenchymal stem cells. Tissue Eng Part A 16: 1009-1019. 
20. Weiss S, Hennig T, Bock R, Steck E, Richter W (2010) Impact of growth factors and PTHrP on early and late chondrogenic differentiation of human mesenchymal stem cells. J Cell Physiol 223: 84-93.

21. Longobardi L, O'Rear L, Aakula S, Johnstone B, Shimer K, et al. (2006) Effect of IGF-I in the chondrogenesis of bone marrow mesenchymal stem cells in the presence or absence of TGF-beta signaling. J Bone Miner Res 21: 626-636.

22. Chen Y, Bloemen V, Impens S, Moesen M, Luyten FP, et al. (2011) Characterization and optimization of cell seeding in scaffolds by factorial design: quality by design approach for skeletal tissue engineering. Tissue Eng Part C Methods 17: 12111221.

23. Liu G, Kawaguchi H, Ogasawara T, Asawa Y, Kishimoto J, et al. (2007) Optimal combination of soluble factors for tissue engineering of permanent cartilage from cultured human chondrocytes. J Biol Chem 282: 20407-20415.

24. Enochson L, Brittberg M, Lindahl A (2012) Optimization of a chondrogenic medium through the use of factorial design of experiments. Biores Open Access 1: 306313.

25. Geiss GK, Bumgarner RE, Birditt B, Dahl T, Dowidar N, et al. (2008) Direct multiplexed measurement of gene expression with color-coded probe pairs. Nature Biotechnology 26: 317-325.

26. Malkov VA, Serikawa KA, Balantac N, Watters J, Geiss G, et al. (2009) Multiplexed measurements of gene signatures in different analytes using the Nanostring nCounter Assay System. BMC Res Notes 2: 80.

27. Shahdadfar A, Fronsdal K, Haug T, Reinholt FP, Brinchmann JE (2005) In vitro expansion of human mesenchymal stem cells: choice of serum is a determinant of cell proliferation, differentiation, gene expression, and transcriptome stability. Stem Cells 23: 1357-1366.

28. Herlofsen SR, Kuchler AM, Melvik JE, Brinchmann JE (2011) Chondrogenic differentiation of human bone marrow-derived mesenchymal stem cells in selfgelling alginate discs reveals novel chondrogenic signature gene clusters. Tissue Eng Part A 17: 1003-1013. 
29. NanoString Technologies (2012) nCounter Expression Data Analysis Guide.

NanoString Technologies, Inc.

http://www.nanostring.com/media/pdf/MAN_nCounter_Gene_Expression_Data Analysis_Guidelines.pdf. Accessed: June 13th, 2012.

30. Andersen CL, Jensen JL, Orntoft TF (2004) Normalization of real-time quantitative reverse transcription-PCR data: a model-based variance estimation approach to identify genes suited for normalization, applied to bladder and colon cancer data sets. Cancer Res 64: 5245-5250.

31. Huber W, von Heydebreck A, Sultmann H, Poustka A, Vingron M (2002) Variance stabilization applied to microarray data calibration and to the quantification of differential expression. Bioinformatics 18 Suppl 1: S96-104.

32. Smyth GK (2005) Limma: linear models for microarray data. In: Gentleman R, Carey V, Dudoit S, Irizarry R, Huber W, editors. Bioinformatics and Computational Biology Solutions using R and Bioconductor. New York: Springer. pp. 397-420.

33. Dray S, Dufour AB (2007) The ade4 package: implementing the duality diagram for ecologists. Journal of Statistical Software 22: 1-20.

34. Bergkvist A, Rusnakova V, Sindelka R, Garda JM, Sjogreen B, et al. (2010) Gene expression profiling--Clusters of possibilities. Methods 50: 323-335.

35. Seibel MJ, Robins SP, Bilezikian JP (2006) Dynamics of bone and cartilage metabolism. San Diego: Academic Press. xix, 919 s. p.

36. Bahney CS, Hsu CW, Yoo JU, West JL, Johnstone B (2011) A bioresponsive hydrogel tuned to chondrogenesis of human mesenchymal stem cells. FASEB J 25: 1486-1496.

37. Huang W, Li WQ, Dehnade F, Zafarullah M (2002) Tissue inhibitor of metalloproteinases-4 (TIMP-4) gene expression is increased in human osteoarthritic femoral head cartilage. J Cell Biochem 85: 295-303.

38. Park JR, Jung JW, Lee YS, Kang KS (2008) The roles of Wnt antagonists Dkk1 and sFRP4 during adipogenesis of human adipose tissue-derived mesenchymal stem cells. Cell Prolif 41: 859-874. 
39. Higashikawa A, Saito T, Ikeda T, Kamekura S, Kawamura N, et al. (2009) Identification of the core element responsive to runt-related transcription factor 2 in the promoter of human type X collagen gene. Arthritis Rheum 60: 166-178.

40. Jay GD, Torres JR, Warman ML, Laderer MC, Breuer KS (2007) The role of lubricin in the mechanical behavior of synovial fluid. Proc Natl Acad Sci U S A 104: 6194-6199.

41. Zimmerman LB, De Jesús-Escobar JM, Harland RM (1996) The Spemann Organizer Signal noggin Binds and Inactivates Bone Morphogenetic Protein 4. Cell 86: 599606.

42. Chen D, Zhao M, Mundy GR (2004) Bone morphogenetic proteins. Growth Factors 22: $233-241$.

43. Oldershaw RA, Tew SR, Russell AM, Meade K, Hawkins R, et al. (2008) Notch signaling through Jagged-1 is necessary to initiate chondrogenesis in human bone marrow stromal cells but must be switched off to complete chondrogenesis. Stem Cells 26: 666-674.

44. Oldershaw RA, Hardingham TE (2010) Notch signaling during chondrogenesis of human bone marrow stem cells. Bone 46: 286-293.

45. Okamoto O, Fujiwara S (2006) Dermatopontin, a novel player in the biology of the extracellular matrix. Connect Tissue Res 47: 177-189.

46. Dehne T, Schenk R, Perka C, Morawietz L, Pruss A, et al. (2010) Gene expression profiling of primary human articular chondrocytes in high-density micromasses reveals patterns of recovery, maintenance, re- and dedifferentiation. Gene 462: 817.

47. Sekiya I, Vuoristo JT, Larson BL, Prockop DJ (2002) In vitro cartilage formation by human adult stem cells from bone marrow stroma defines the sequence of cellular and molecular events during chondrogenesis. Proc Natl Acad Sci U S A 99: 43974402.

48. Decaris ML, Leach JK (2011) Design of experiments approach to engineer cellsecreted matrices for directing osteogenic differentiation. Ann Biomed Eng 39: 1174-1185. 
49. Hunt MM, Meng G, Rancourt DE, Gates ID, Kallos MS (2013) Factorial

Experimental Design for the Culture of Human Embryonic Stem Cells as Aggregates in Stirred Suspension Bioreactors Reveals the Potential for Interaction Effects Between Bioprocess Parameters. Tissue Eng Part C Methods Online Ahead of Print, Jul 16.

50. Buxton AN, Bahney CS, Yoo J, Johnstone B (2010) Temporal Exposure to Chondrogenic Factors Modulates Human Mesenchymal Stem Cell Chondrogenesis in Hydrogels. Tissue Eng Part A.

51. Craft AM, Ahmed N, Rockel JS, Baht GS, Alman BA, et al. (2013) Specification of chondrocytes and cartilage tissues from embryonic stem cells. Development 140: 2597-2610.

52. Schmitt B, Ringe J, Haupl T, Notter M, Manz R, et al. (2003) BMP2 initiates chondrogenic lineage development of adult human mesenchymal stem cells in high-density culture. Differentiation 71: 567-577.

53. Keller B, Yang T, Chen Y, Munivez E, Bertin T, et al. (2011) Interaction of TGFbeta and BMP signaling pathways during chondrogenesis. PLoS One 6: e16421.

54. Hildner F, Peterbauer A, Wolbank S, Nurnberger S, Marlovits S, et al. (2010) FGF-2 abolishes the chondrogenic effect of combined BMP-6 and TGF-beta in human adipose derived stem cells. J Biomed Mater Res A 94: 978-987.

55. Buckley CT, Kelly DJ (2012) Expansion in the presence of FGF-2 enhances the functional development of cartilaginous tissues engineered using infrapatellar fat pad derived MSCs. J Mech Behav Biomed Mater 11: 102-111.

56. Luyten FP, Hascall VC, Nissley SP, Morales TI, Reddi AH (1988) Insulin-like growth factors maintain steady-state metabolism of proteoglycans in bovine articular cartilage explants. Archives of Biochemistry and Biophysics 267: 416425 .

57. van Kleffens M, Groffen C, Rosato RR, van den Eijnde SM, van Neck JW, et al. (1998) mRNA expression patterns of the IGF system during mouse limb bud development, determined by whole mount in situ hybridization. Molecular and Cellular Endocrinology 138: 151-161. 
58. Worster AA, Brower-Toland BD, Fortier LA, Bent SJ, Williams J, et al. (2001)

Chondrocytic differentiation of mesenchymal stem cells sequentially exposed to transforming growth factor- $\beta 1$ in monolayer and insulin-like growth factor-I in a three-dimensional matrix. Journal of Orthopaedic Research 19: 738-749.

59. Kawamura K, Chu CR, Sobajima S, Robbins PD, Fu FH, et al. (2005) Adenoviralmediated transfer of TGF-beta1 but not IGF-1 induces chondrogenic differentiation of human mesenchymal stem cells in pellet cultures. Exp Hematol 33: $865-872$.

60. Schwanhausser B, Busse D, Li N, Dittmar G, Schuchhardt J, et al. (2011) Global quantification of mammalian gene expression control. Nature 473: 337-342.

61. Mandenius CF, Brundin A (2008) Bioprocess optimization using design-ofexperiments methodology. Biotechnol Prog 24: 1191-1203.

62. Bock HC, Michaeli P, Bode C, Schultz W, Kresse H, et al. (2001) The small proteoglycans decorin and biglycan in human articular cartilage of late-stage osteoarthritis. Osteoarthritis Cartilage 9: 654-663.

63. Roughley PJ (2006) The structure and function of cartilage proteoglycans. Eur Cell Mater 12: 92-101.

64. Zhu F, Friedman MS, Luo W, Woolf P, Hankenson KD (2012) The transcription factor osterix (SP7) regulates BMP6-induced human osteoblast differentiation. J Cell Physiol 227: 2677-2685.

65. Bode-Lesniewska B, Dours-Zimmermann MT, Odermatt BF, Briner J, Heitz PU, et al. (1996) Distribution of the large aggregating proteoglycan versican in adult human tissues. Journal of Histochemistry \& Cytochemistry 44: 303-312.

66. Binette F, McQuaid DP, Haudenschild DR, Yaeger PC, McPherson JM, et al. (1998) Expression of a stable articular cartilage phenotype without evidence of hypertrophy by adult human articular chondrocytes in vitro. J Orthop Res 16 : 207-216. 


\section{Figure legends}

\section{Figure 1: Experimental setup and principal component analysis (PCA)}

A. Experimental setup with numbering of the different conditions. When not stated, the cell density was $10^{7}$ cells per mL. B. PCA on all conditions labelled by days in culture. C. PCA limited to conditions $1-32$ on days 0 and 1. D. PCA limited to conditions 1-32 on days 0 and day 7 .

\section{Figure 2: Statistical analysis of main effects and interactions at day 7}

A. Normal plot of the standardized effects with the response set to mean expression of wanted markers. B. Corresponding main effects plot of all factors. C and D. Corresponding plots of significant second order interactions. E. Normal plot of the standardized effects with the response set to mean expression of unwanted markers. F. Corresponding main effects plot of all factors. $\mathbf{G}$ and $\mathbf{H}$. Corresponding plots of significant second order interactions.

\section{Figure 3: Analysis of wanted and unwanted gene expression}

A. Gene sets used to compute mean expression of wanted and unwanted markers. B. Scatter-plot of the mean expression (studentized values) of wanted (x-axis) and unwanted (y-axis) markers at day 0,1 and 7 of all conditions. C. Scatter-plot of the mean expression (studentized values) of wanted and unwanted markers at day 0 and 7 for condition $1-32$. 


\section{Figure 4: Heatmap of top ranking conditions.}

Heatmap of wanted and unwanted genes in all conditions significantly enriched for wanted, but not unwanted markers, color coded by the studentized score.

\section{Figure 5: Genes uniformly affected by single factors across conditions 1 - 32 at day 7.}
A. Experimental setup conditions 1 - 32. B. Heatmap of genes significantly downregulated in all conditions contaning any one of the factors compared to condition 32. C. Heatmap of genes significantly upregulated in all conditions contaning any one of the factors compared to condition 32. Values are $\log 2$-transformed mean expressions $(n=$ 3)

Figure 6: Genes significantly regulated between key conditions (day 7)

A. Top 20 upregulated and top 20 downregulated genes when adding DEX to TGFB1. B. Top 20 upregulated and top 20 downregulated genes when adding DEX to

TGFB1+BMP2. C. All regulated genes when adding BMP2 to TGFB1. D. All regulated genes when adding BMP2 to TGFB1+DEX.

Values represent $\log 2$ to the fold change between the gene expression in the condition without and the condition with the specified factor added.

\section{Supplementary Figure 1: Quality control of MSCs}

A. Light microscopy picture of MSCs and surface marker profiles of MSCs as measured by flow cytometry before differentiation (passage 2 or 3). B. Light microscopy pictures of stained control (CTRL) and differentiated (DIFF) MSCs on day 21. Upper panel; 
adipogenic differentiated cells stained with Oil Red-O: Lower panel: osteogenic differentiation of MSCs stained with Alizarin Red. C. Fold change of the expression of PPARG in adipogenic differentiated cells and OMD in osteogenic differentiated relative to control treated cells (mean $\pm \mathrm{SE}, \mathrm{n}=3$ ). D. Chondrogenically differentiated MSCs in alginate with TGFB1, DEX and BMP2 as shown by mRNA expression changes in key chondrogenic markers $(n=3$, mean \pm SE) with corresponding protein synthesis $(\mathbf{E}$.) in a representative sample on day 21. Nuclei counterstained with DAPI (blue). Scale bar $=50$ $\mu \mathrm{M}$.

Supplementary Figure S2: Correlation between gene expression analyzed in lysates and in isolated total RNA from corresponding samples. Correlation plots of the expression of genes analyzed in lysates and in total RNA in two donors at day 1, 7 and 14 with coefficients of determination (Pearson's correlation).

\title{
Supplementary Figure S3: Statistical analysis of main effects and interactions at day
}

1.

\begin{abstract}
A. Normal plot of the standardized effects with response set to mean expression of wanted markers. B. Corresponding main effects plot of all factors. C. Corresponding plots of significant second order interactions. D. Normal plot of the standardized effects with response set to mean expression of unwanted markers. E. Corresponding main effects plot of all factors. F. Corresponding plots of significant second order interactions.
\end{abstract}

\section{Supplementary Figure S4: Analysis of wanted and unwanted gene expression}


A. Scatter-plot of the mean expression (studentized values) of wanted and unwanted markers at day 0 and 7 for conditions containing isoforms of TGFB $(n=2)$. B. Scatterplot of the mean expression (studentized values) of wanted and unwanted markers at day 0 and 7 for conditions with isoforms of BMP. $(n=2)$ B. Scatter-plot of the mean expression (studentized values) of wanted and unwanted markers at day 0 and 7 for condition with varying cell seeding density. $(n=2)$

\section{Supplementary Figure S5: Genes significantly up- or downregulated}

Heatmap of all genes significantly changed compared to condition 32 at day 7 . Values are $\log 2$-transformed mean expressions $(\mathrm{n}=3)$

\section{Supplementary Figure S6: Genes significantly regulated between key conditions} (day 7)

Upper panel: All significantly regulated genes (>2-fold) when adding DEX to TGFB1. Lower panel: All significantly regulated genes ( $>2$-fold) when adding DEX to TGFB1+BMP2. Values represent $\log 2$ to the fold change between the gene expression in the condition without and the condition with the specified factor added.

\section{Supplementary Figure S7: Overlapping regulated genes when adding DEX to TGF or to TGFB1 with BMP2. \\ Venn diagram illustrating the overlap of genes significantly up- or downregulated corresponding to Supplementary Figure S6.}


Table 1: Selected wanted and unwanted gene sets.

\begin{tabular}{|c|c|c|c|}
\hline & Gene symbol & Gene name & Functional role \\
\hline \multirow[t]{11}{*}{ WANTED } & $A C A N$ & aggrecan & Major proteoglycan in hyaline cartilage \\
\hline & $B G N$ & biglycan & $\begin{array}{l}\text { Small leucine rich proteoglycans, pericellular } \\
\text { location and links to chondroitin sulfate in } \\
\text { hyaline cartilage }\end{array}$ \\
\hline & COL11A1/2 & $\begin{array}{l}\text { collagen, type XI, } \\
\text { alpha } 1 / 2\end{array}$ & $\begin{array}{l}\text { Fibril forming collagen found associated with } \\
\text { type } 2 \text { collagen in hyaline cartilage }\end{array}$ \\
\hline & COL2A1 & $\begin{array}{l}\text { collagen, type II, } \\
\text { alpha } 1\end{array}$ & $\begin{array}{l}\text { The major fibril forming collagen almost } \\
\text { exclusively found in hyaline cartilage }\end{array}$ \\
\hline & COL9A1/2/3 & $\begin{array}{l}\text { collagen, type IX, } \\
\text { alpha } 1 / 2 / 3\end{array}$ & $\begin{array}{l}\text { Fibril associated collagen with interrupted } \\
\text { triple helix found covalently linked to } \\
\text { collagen type } 2 \text { in hyaline cartilage }\end{array}$ \\
\hline & COMP & $\begin{array}{l}\text { cartilage oligomeric } \\
\text { matrix protein }\end{array}$ & $\begin{array}{l}\text { Prominent component in the ECM of hyaline } \\
\text { cartilage possibly stabilizing the collagen } \\
\text { fibril assembly and network }\end{array}$ \\
\hline & $D C N$ & decorin & $\begin{array}{l}\text { Small leucine rich proteoglycan, binds to } \\
\text { collagen fibrils and aids in assembly }\end{array}$ \\
\hline & $F M O D$ & fibromodulin & $\begin{array}{l}\text { Small leucine rich proteoglycan, aids in } \\
\text { collagen assembly in cartilage in early } \\
\text { development }\end{array}$ \\
\hline & HAPLN1 & $\begin{array}{l}\text { hyaluronan and } \\
\text { proteoglycan link } \\
\text { protein } 1\end{array}$ & $\begin{array}{l}\text { Abundant protein in cartilage, stabilizes } \\
\text { aggregates of hyaluronan and aggrecan }\end{array}$ \\
\hline & $L U M$ & lumican & $\begin{array}{l}\text { Leucine rich proteoglycan, aids in collagen } \\
\text { assembly in cartilage in early development }\end{array}$ \\
\hline & MATN3 & matrilin 3 & $\begin{array}{l}\text { Matrix protein restricted to cartilage and } \\
\text { binds tightly to aggrecan and/collagen fibrils }\end{array}$ \\
\hline \multirow[t]{9}{*}{ UNWANTED } & $A L P L$ & $\begin{array}{l}\text { alkaline } \\
\text { phosphatase, } \\
\text { liver/bone/kidney }\end{array}$ & $\begin{array}{l}\text { Major enzyme leading to mineralization of } \\
\text { bone }\end{array}$ \\
\hline & COL10A1 & $\begin{array}{l}\text { collagen type } X \text {, } \\
\text { alpha } 1\end{array}$ & $\begin{array}{l}\text { Network forming collagen found } \\
\text { predominantly in hypertrophic or diseased } \\
\text { cartilage }\end{array}$ \\
\hline & COL1A1/2 & $\begin{array}{l}\text { collagen type I, } \\
\text { alpha } 1 / 2\end{array}$ & $\begin{array}{l}\text { Fibril forming cartilage abundant in bone } \\
\text { ECM and virtually absent in hyaline cartilage }\end{array}$ \\
\hline & COL3A1 & $\begin{array}{l}\text { collagen type III, } \\
\text { alpha } 1\end{array}$ & $\begin{array}{l}\text { Fibril forming collagen often found in mixed } \\
\text { fibrils with collagen type } 1\end{array}$ \\
\hline & $O G N$ & osteoglycin & $\begin{array}{l}\text { Small leucine rich proteoglycan, induces } \\
\text { bone formation }\end{array}$ \\
\hline & PPARG & $\begin{array}{l}\text { peroxisome } \\
\text { proliferator- } \\
\text { activated receptor } \\
\text { gamma }\end{array}$ & $\begin{array}{l}\text { Nuclear receptor, promotes adipogenesis, } \\
\text { stimulates lipid uptake and glucose } \\
\text { metabolism }\end{array}$ \\
\hline & $R U N X 2$ & $\begin{array}{l}\text { runt related } \\
\text { transcription factor } \\
2\end{array}$ & $\begin{array}{l}\text { Transcription factor required for bone } \\
\text { formation }\end{array}$ \\
\hline & $S P 7$ & osterix & $\begin{array}{l}\text { Transcription factor essential for } \\
\text { osteoblastogenesis }\end{array}$ \\
\hline & SPP1 & osteopontin & Bone protein, potentiates osteoclast adhesion \\
\hline
\end{tabular}




\begin{tabular}{|l|l|l|l|}
\hline & & & to mineral surfaces \\
\hline & $V C A N$ & versican & $\begin{array}{l}\text { Proteoglycan present in fibrous and elastic } \\
\text { cartilage, upregulated in dedifferentiating } \\
\text { chondrocytes }\end{array}$ \\
\hline
\end{tabular}

Gene symbol and name of all genes comprising the wanted and unwanted marker gene sets. Based on references [35,62-66] 
Table 2: Gene set enrichment analysis for wanted and unwanted markers.

\begin{tabular}{|r|c|r|}
\hline & \multicolumn{2}{|c|}{ False discovery rate } \\
\hline Condition & Wanted & Unwanted \\
\hline $\mathbf{1 1}$ & $<\mathbf{0 . 0 0 0 1}$ & $\mathbf{0 . 4 5 2}$ \\
\hline $\mathbf{1 0}$ & $<\mathbf{0 . 0 0 0 1}$ & $\mathbf{0 . 2 7 3}$ \\
\hline $\mathbf{9}$ & $<\mathbf{0 . 0 0 0 1}$ & $\mathbf{0 . 4 8 4}$ \\
\hline $\mathbf{1 2}$ & $<\mathbf{0 . 0 0 0 1}$ & $\mathbf{0 . 2 9 6}$ \\
\hline $\mathbf{1}$ & $<\mathbf{0 . 0 0 0 1}$ & $\mathbf{0 . 1 3 5}$ \\
\hline $\mathbf{1 3}$ & $<\mathbf{0 . 0 0 0 1}$ & $\mathbf{0 . 1 3 8}$ \\
\hline $\mathbf{4}$ & $<\mathbf{0 . 0 0 0 1}$ & $\mathbf{0 . 0 5 2}$ \\
\hline $\mathbf{2}$ & $<\mathbf{0 . 0 0 0 1}$ & $\mathbf{0 . 0 8 0}$ \\
\hline $\mathbf{5}$ & $\mathbf{0 . 0 0 3}$ & $\mathbf{0 . 0 5 6}$ \\
\hline $\mathbf{7}$ & $\mathbf{0 . 0 3 5}$ & $\mathbf{0 . 0 6 7}$ \\
\hline 15 & $<0.0001$ & $\mathbf{0 . 0 5 1}$ \\
\hline 14 & 0.001 & 0.044 \\
\hline 19 & 0.017 & 0.014 \\
\hline 17 & 0.020 & 0.018 \\
\hline 29 & 0.030 & 0.016 \\
\hline 31 & 0.033 & 0.023 \\
\hline & & 0.023 \\
\hline
\end{tabular}

All conditions enriched for wanted markers are listed and ranked by the false discovery rate for wanted markers (exact FDR values are not stated for FDR $<0.0001$ ). Conditions enriched for wanted but not for unwanted markers are marked in bold and stated first. 
Figure 1: Experimental setup and principal component analysis (PCA)

A

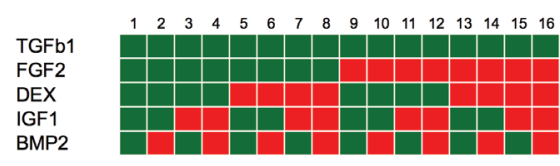

$\begin{array}{llllllllllllllll}17 & 18 & 19 & 20 & 21 & 22 & 23 & 24 & 25 & 26 & 27 & 28 & 29 & 30 & 31 & 32\end{array}$

FGF2

DEX

IGF1

BMP2

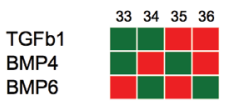

TGFb1

DEX

BMP2

Cell density $1,2 \quad 2,5 \quad 1020$

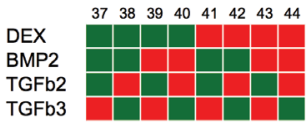

TGFb2
TGFb3
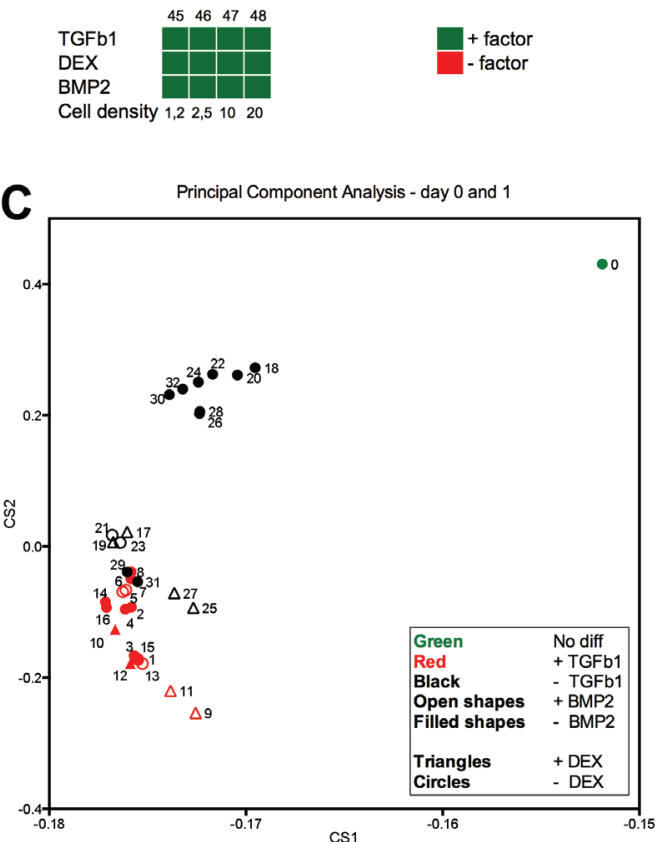
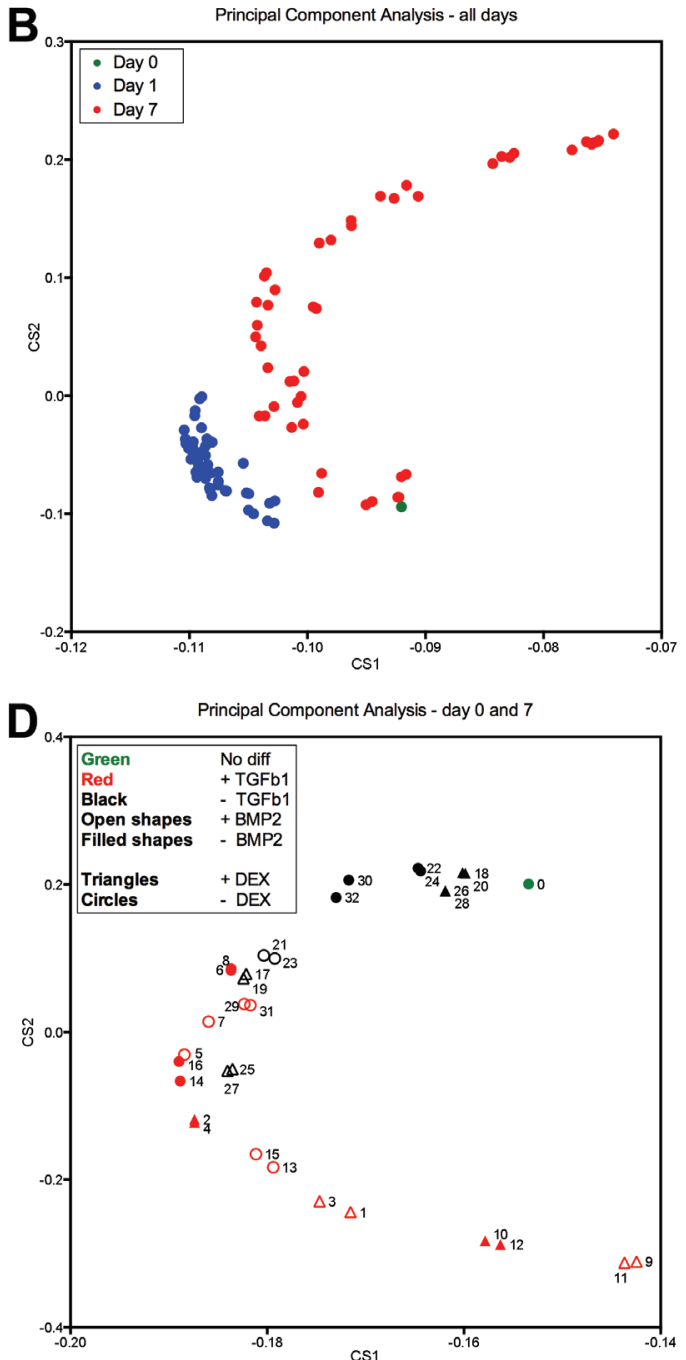

A. Experimental setup with numbering of the different conditions. When not stated, the cell density was 107 cells per mL. B. PCA on all conditions labelled by days in culture. C. PCA limited to conditions $1-32$ on days 0 and 1. D. PCA limited to conditions 1-32 on days 0 and day 7. 
Figure 2: Statistical analysis of main effects and interactions at day 7

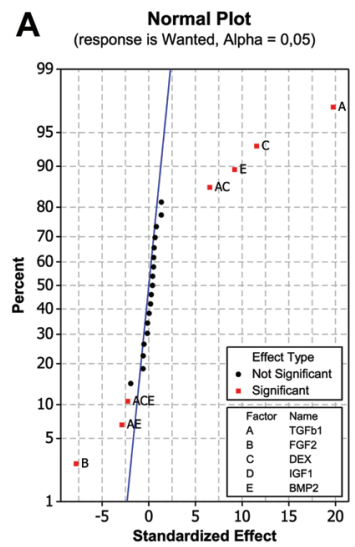

B
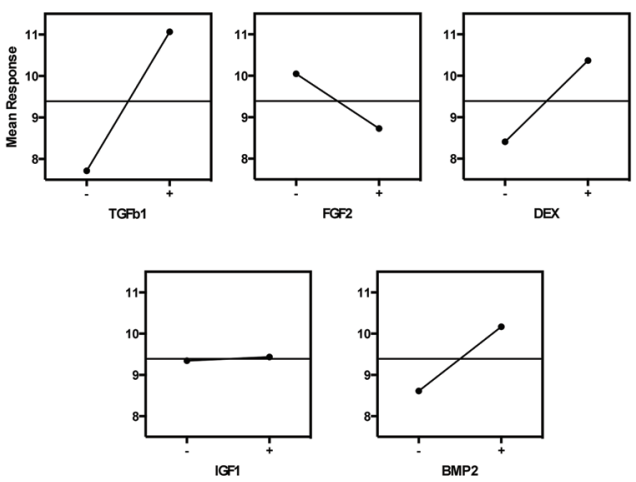

$\mathbf{F}$

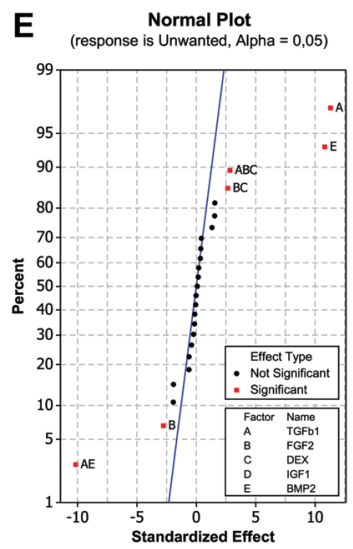

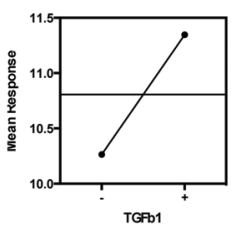

TGF1

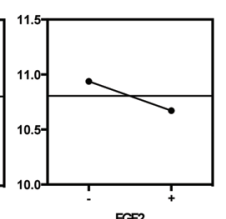

FGF2
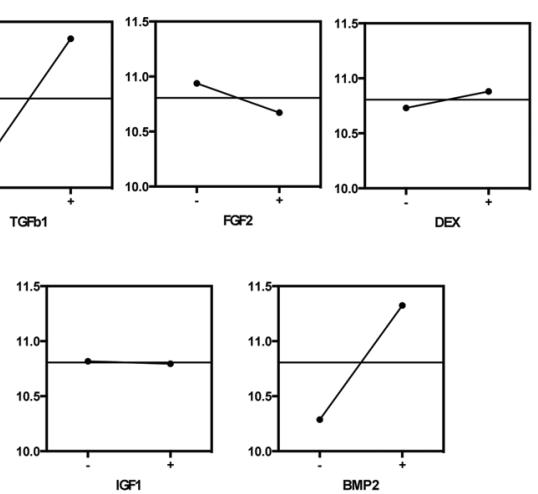

D

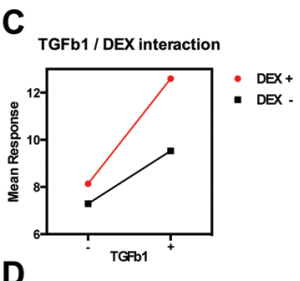

TGFb1 / BMP2 interaction

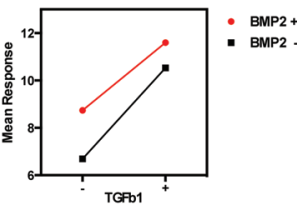

G

TGFb1 / BMP2 interaction

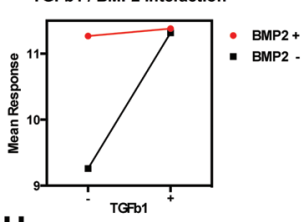

H

FGF2 / DEX interaction

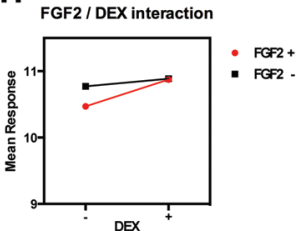

A. Normal plot of the standardized effects with the response set to mean expression of wanted markers. B. Corresponding main effects plot of all factors. C and D.

Corresponding plots of significant second order interactions. E. Normal plot of the standardized effects with the response set to mean expression of unwanted markers. F. Corresponding main effects plot of all factors. $\mathrm{G}$ and $\mathrm{H}$. Corresponding plots of significant second order interactions. 
Figure 3: Analysis of wanted and unwanted gene expression

A
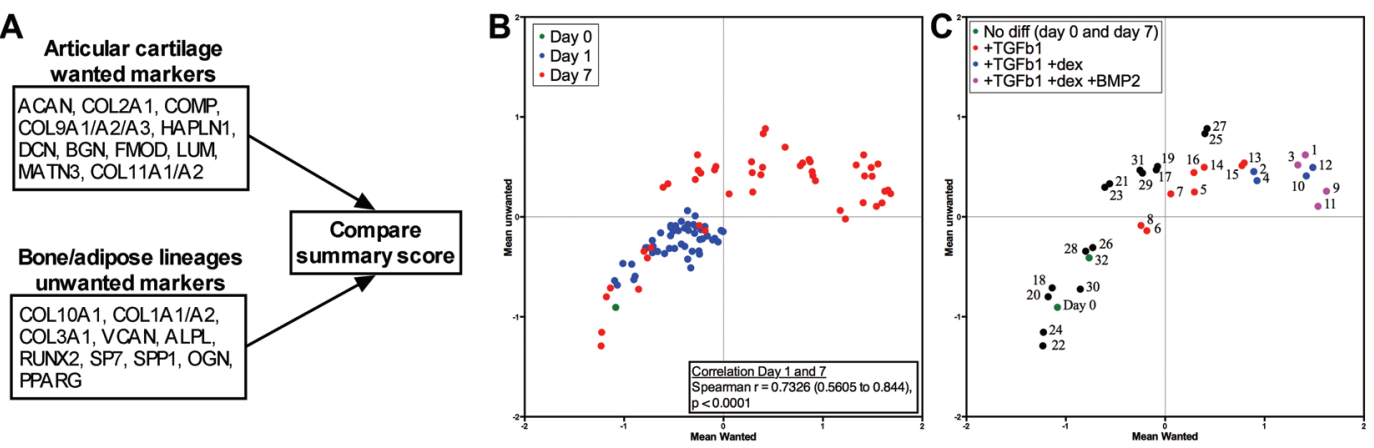

A. Gene sets used to compute mean expression of wanted and unwanted markers. B. Scatter-plot of the mean expression (studentized values) of wanted (x-axis) and unwanted (y-axis) markers at day 0 , 1 and 7 of all conditions. C. Scatter-plot of the mean expression (studentized values) of wanted and unwanted markers at day 0 and 7 for condition $1-32$. 
Figure 4: Heatmap of top ranking conditions.

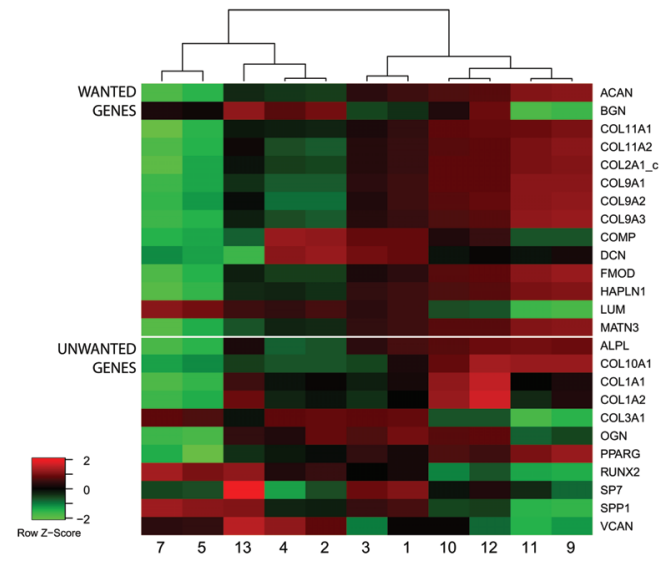

Heatmap of wanted and unwanted genes in all conditions significantly enriched for wanted, but not unwanted markers, color coded by the studentized score. 
Figure 5: Genes uniformly affected by single factors across conditions 1 - 32 at day 7.

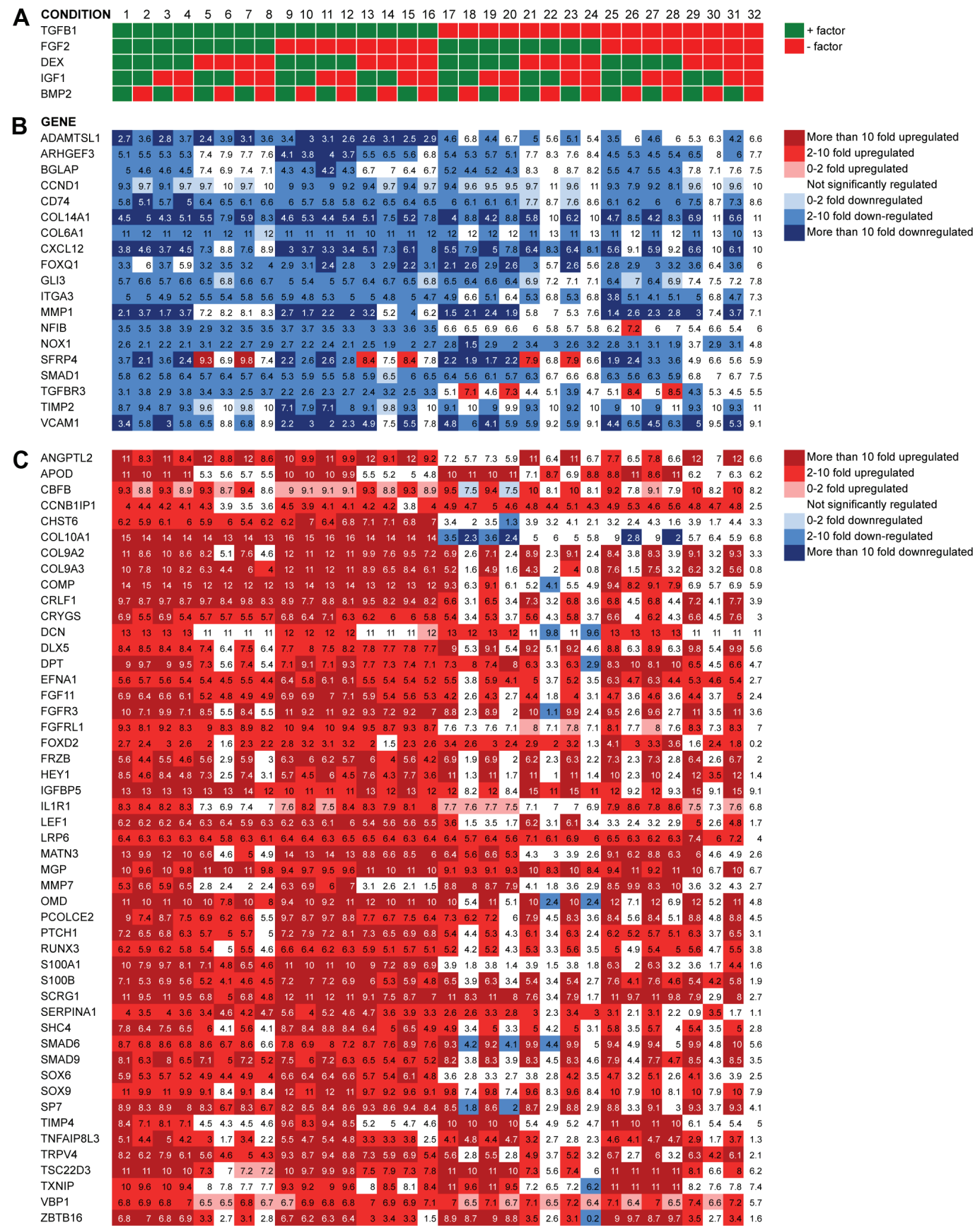

A. Experimental setup conditions 1 - 32. B. Heatmap of genes significantly downregulated in all conditions contaning any one of the factors compared to condition 32. C. Heatmap of genes significantly upregulated in all conditions contaning any one of the factors compared to condition 32 . Values are $\log 2$-transformed mean expressions $(n=3)$ 
Figure 6: Genes significantly regulated between key conditions (day 7)
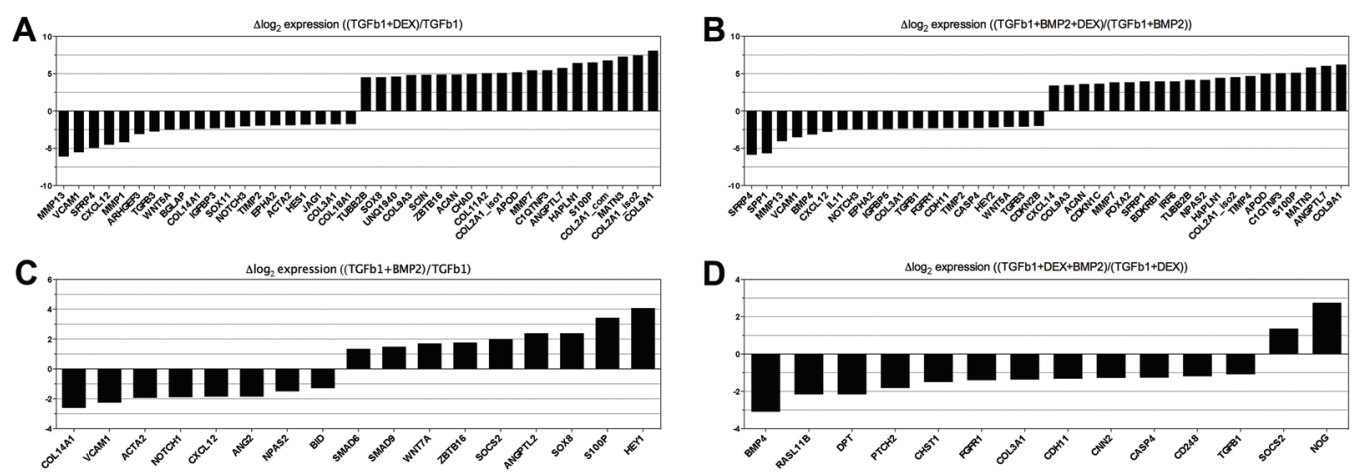

A. Top 20 upregulated and top 20 downregulated genes when adding DEX to TGFB1. B. Top 20 upregulated and top 20 downregulated genes when adding DEX to TGFB1+BMP2. C. All regulated genes when adding BMP2 to TGFB1. D. All regulated genes when adding BMP2 to TGFB1+DEX.

Values represent $\log 2$ to the fold change between the gene expression in the condition without and the condition with the specified factor added. 


\section{Supporting information Methods and materials}

\section{Cell harvest and culture}

Bone marrow aspirates were obtained in heparin-coated syringes and immediately diluted 1:4 with DMEM-F12 (Gibco) and centrifuged on a density gradient (Lymphoprep, Axis Shield, Oslo, Norway). The mononuclear layer was washed twice and seeded into 175 $\mathrm{cm}^{2}$ culture flask (Nunc, Roskilde, Denmark). Cells were allowed to adhere for 72 hours before the first medium change. Medium was then changed every 3-4 days. Cells were passaged when approximately $75 \%$ confluent and reseeded at a density of $5000 \mathrm{cells} / \mathrm{cm}^{2}$.

\section{Medium and supplements}

Human platelet lysate (hPL) was prepared as published previously[1,2]. Briefly, 1 unit of pooled platelets (4 donors) in platelet additive solution was spun at $1700 \mathrm{~g}$ at room temperature. The resulting pellet was resuspended in $10 \mathrm{~mL}$ Octaplas AB (Octapharma AS, Jessheim, Norway) and frozen at $-20^{\circ} \mathrm{C}$. After thawing, platelets from 19 units were pooled and adjusted to a final volume of $4,8 \mathrm{~L}$ with Octaplas $\mathrm{AB}$, subjected to a second freeze-thaw-cycle, centrifuged at $4000 \mathrm{~g}$ at $4^{\circ} \mathrm{C}$ for 15 minutes to remove platelet fragments and frozen in aliquotes.

Validation of cells as MSC

Characterization was performed as previously described[3]. Fluorochrome conjugated antibodies used for surface marker characterization were CD19-APC, CD105-APC, HLA-DR-APC, CD14-FITC (all Diatec, Oslo, Norway), CD34-FITC, CD44-FITC, CD45-PE, CD73-PE (all BD-Biosciences, San Jose, CA) and CD90-PE (Serotec, Oxford, UK) and appropriate IgG control antibodies. Multipotency was validated by osteogenic 
and adipogenic differentiation[4]. For osteogenic differentiation cells were seeded at 3000 cells $/ \mathrm{cm}^{2}$ and differentiated with DMEM-F12 containing $10 \% \mathrm{hPL}, 10 \mathrm{mM} \beta$ glycerophosphate, $100 \mathrm{nM}$ DEX and $0.05 \mathrm{mM}$ ascorbic acid-2-phosphate. Medium was changed twice a week. For adipogenic differentiation cells were seeded at 50,000 cells $/ \mathrm{cm}^{2}$ and differentiated with DMEM-F12 containing $10 \mu \mathrm{g} / \mathrm{mL}$ insulin (Novo Nordisk, Bagsvaerd, Denmark), 0.5 $\mu \mathrm{M}$ 1-methyl-3-isobutylxanthine, $1 \mu \mathrm{M}$ DEX and $100 \mu \mathrm{m}$ indomethacin (Dumex-Alpharma, Copenhagen, Denmark). Medium was changed twice a week. For both differentiation assays, after 3 weeks, the cells were washed with phosphate-buffered saline (PBS), fixed for $1 \mathrm{~h}$ with $4 \%$ paraformaldehyde, and rinsed with PBS. Mineralisation of osteogenic cultures was confirmed by staining with $40 \mathrm{mM}$ Alizarin Red (ph 4.2) for 5 min. Lipid droplets in adipogenic cultures were confirmed by staining with Oil Red O for 10 min. Appropiate control cultures receiving standard growth medium were also stained and evaluated.

\section{$3 D$ cell culture}

Briefly, cells were resuspended into a $1 \%$ Pronova-LVG solution. $62,5 \mu \mathrm{L}$ were transferred to 16-well chamberslides (Labtek, Nunc, Denmark) and mixed with an equal volume of $1 \%$ Pronova-calcium-alginate containing slow releasing calcium particles, giving a final cell density of $1 \times 10^{7}$ cells $/ \mathrm{mL}$. After stabilisation for 15 minutes, discs were transferred to 24-well culture plates (Nunc) where gelling was consolidated by washing with $50 \mathrm{mM} \mathrm{SrCl}_{2}$, followed by three times of washing with regular DMEM before addition of bCDM with or without additional growth factors according to the experimental factorial design. Medium was changed three times per week. 


\section{Immunohistochemical stainings}

Primary and secondary antibodies and all final concentrations are specified in Table S4. Formalin-fixed, paraffin-embedded samples of cells in alginate were sectioned and deparaffinized using standard laboratory procedures and postfixed for 10 minutes in $4 \%$ paraformaldehyde in PBS. Tissue sections were boiled for 20 minutes in $0.05 \%$ citraconic anhydride in $\mathrm{ddH} 2 \mathrm{O}(\mathrm{pH} 7.4)$, incubated with primary antibodies diluted in PBS/1.25\% bovine serum albumin with $0.1 \%$ saponin for permeabilization overnight at $4{ }^{\circ} \mathrm{C}$, followed by secondary reagents for 1.5 hour at room temperature. Stained sections were mounted using ProLong Gold antifading reagent with DAPI (Invitrogen, Carlsbad, CA). Microscopy was performed with a Nikon Eclipse E-600 fluorescence microscope equipped with Nikon Plan-Fluor objective lenses and a Color View III digital camera controlled by Cell-B software (Olympus, Center Valley, PA).

\section{REFERENCES}

1. Schallmoser K, Strunk D (2009) Preparation of pooled human platelet lysate (pHPL) as an efficient supplement for animal serum-free human stem cell cultures. J Vis Exp.

2. Karlsen TA, Jakobsen RB, Mikkelsen TS, Brinchmann JE (2013) microRNA-140 targets RALA and regulates chondrogenic differentiation of human mesenchymal stem cells by translational enhancement of SOX9 and ACAN. Stem Cells Dev.

3. Herlofsen SR, Kuchler AM, Melvik JE, Brinchmann JE (2011) Chondrogenic differentiation of human bone marrow-derived mesenchymal stem cells in selfgelling alginate discs reveals novel chondrogenic signature gene clusters. Tissue Eng Part A 17: 1003-1013.

4. Shahdadfar A, Fronsdal K, Haug T, Reinholt FP, Brinchmann JE (2005) In vitro expansion of human mesenchymal stem cells: choice of serum is a determinant of 
cell proliferation, differentiation, gene expression, and transcriptome stability.

Stem Cells 23: 1357-1366.

p. 4 


\section{Supplementary Figure 1: Quality control of MSCs}

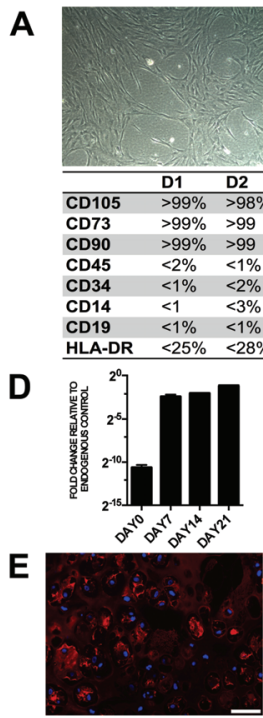

ACAN

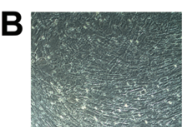

CTRL
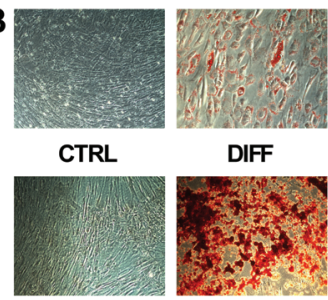

DIFF
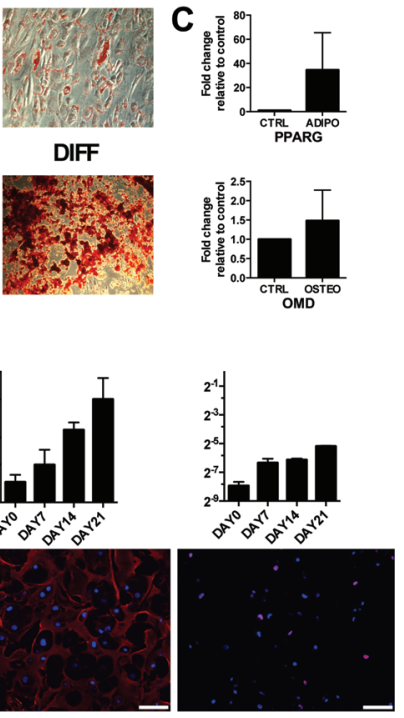

SoX9

A. Light microscopy picture of MSCs and surface marker profiles of MSCs as measured by flow cytometry before differentiation (passage 2 or 3). B. Light microscopy pictures of stained control (CTRL) and differentiated (DIFF) MSCs on day 21. Upper panel;

p. 30

adipogenic differentiated cells stained with Oil Red-O: Lower panel: osteogenic differentiation of MSCs stained with Alizarin Red. C. Fold change of the expression of PPARG in adipogenic differentiated cells and OMD in osteogenic differentiated relative to control treated cells (mean $\pm \mathrm{SE}, \mathrm{n}=3$ ). D. Chondrogenically differentiated MSCs in alginate with TGFB1, DEX and BMP2 as shown by mRNA expression changes in key chondrogenic markers $(n=3$, mean \pm SE) with corresponding protein synthesis (E.) in a representative sample on day 21. Nuclei counterstained with DAPI (blue). Scale bar $=50 \mu \mathrm{M}$. 
Supplementary Figure S2: Correlation between gene expression analyzed in lysates and in isolated total RNA from corresponding samples.

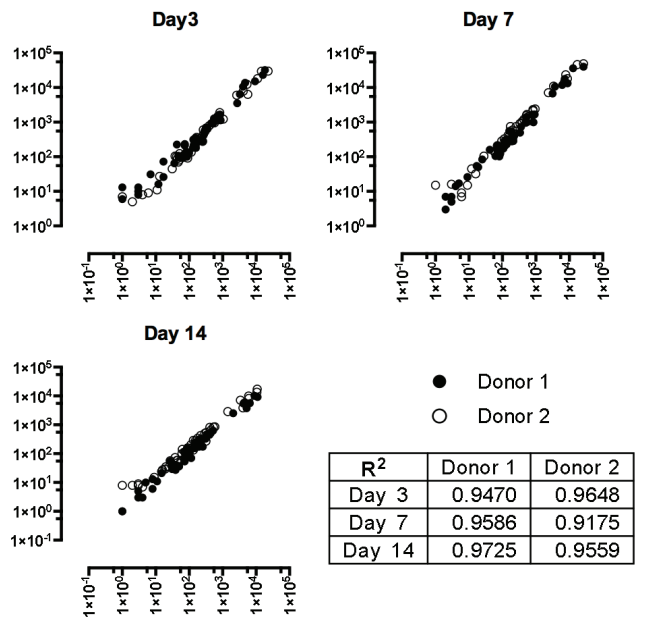

Correlation plots of the expression of genes analyzed in lysates and in total RNA in two donors at day 1, 7 and 14 with coefficients of determination (Pearson's correlation). 
Supplementary Figure S3: Statistical analysis of main effects and interactions at day 1.

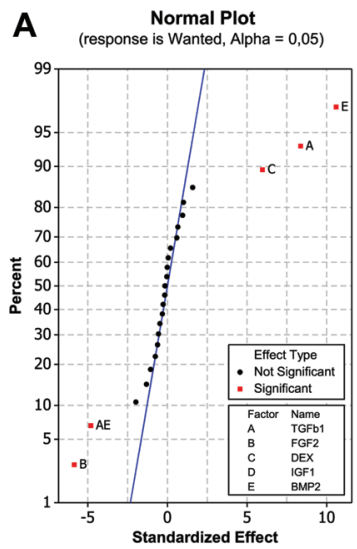

B

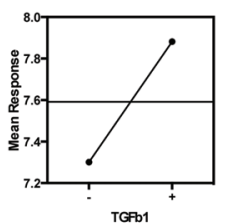

TGFb1

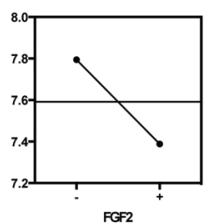

FGF2

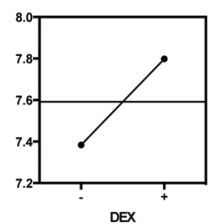

DEX c

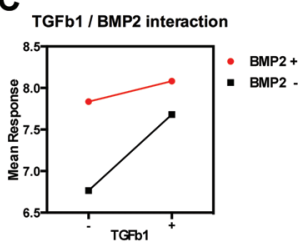

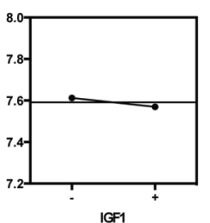

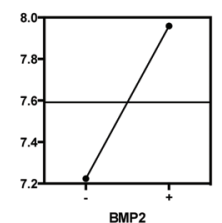

$\mathbf{E}$
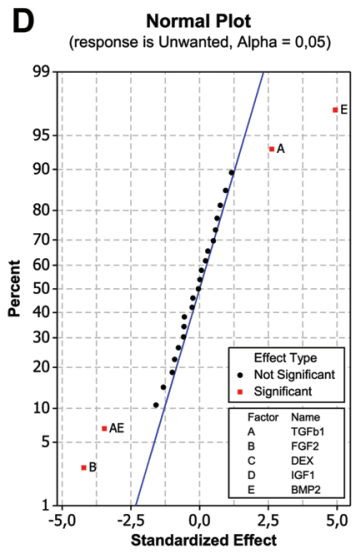

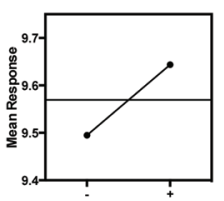

TGFb1

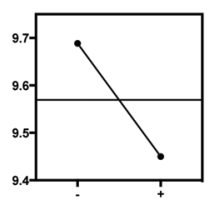

FG2

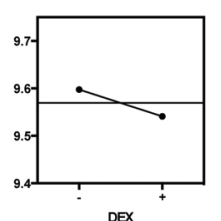

DEX
$\mathbf{F}$

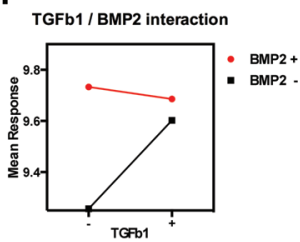

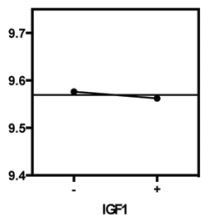

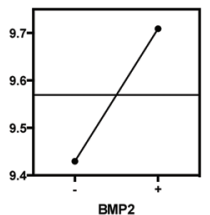

A. Normal plot of the standardized effects with response set to mean expression of wanted markers.

B. Corresponding main effects plot of all factors. C. Corresponding plots of significant second order interactions. D. Normal plot of the standardized effects with response set to mean expression of unwanted markers. E. Corresponding main effects plot of all factors. F. Corresponding plots of significant second order interactions. 
Supplementary Figure S4: Analysis of wanted and unwanted gene expression
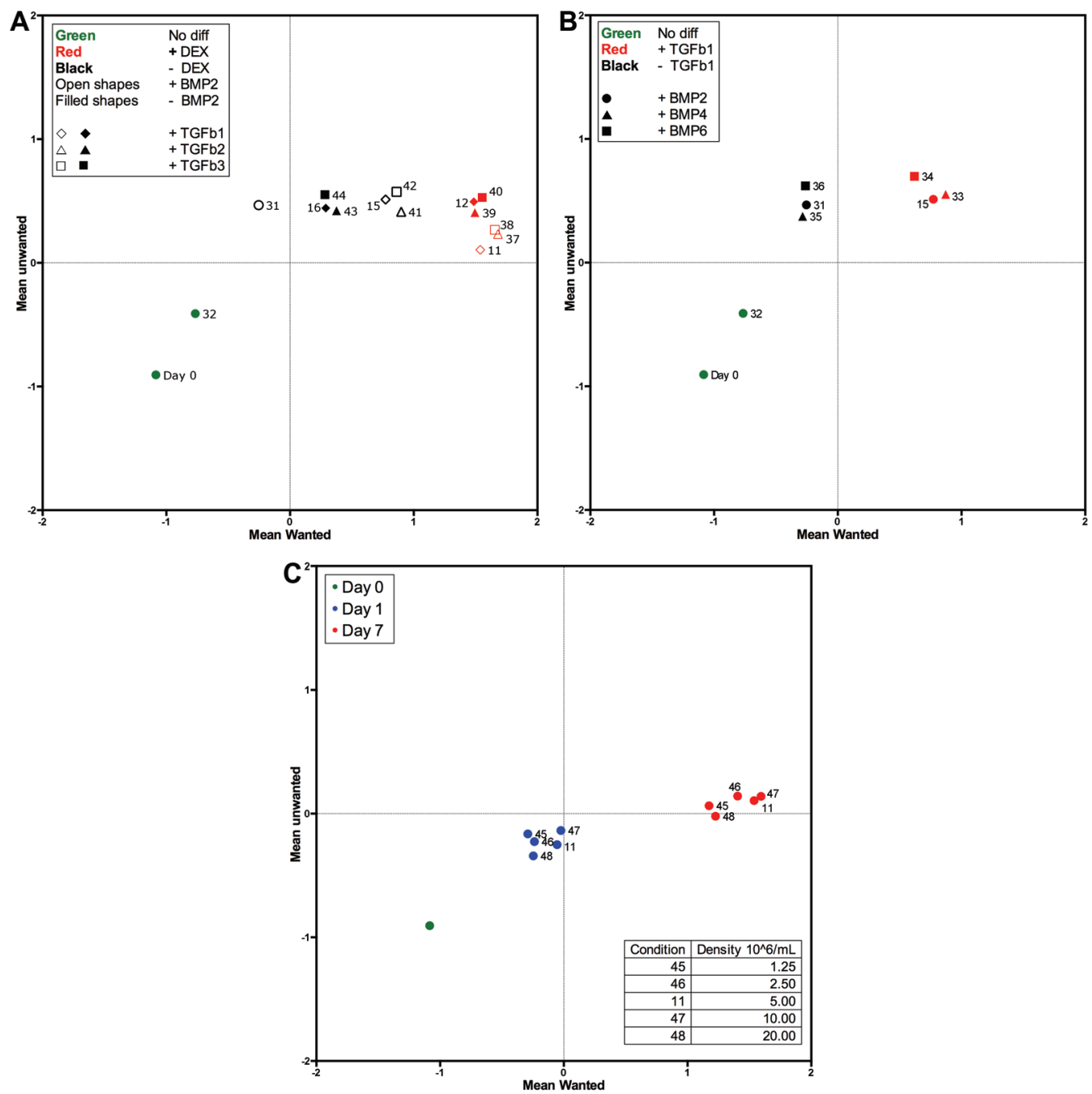

A. Scatter-plot of the mean expression (studentized values) of wanted and unwanted markers at day 0 and 7 for conditions containing isoforms of TGFB $(n=2)$. B. Scatter- plot of the mean expression (studentized values) of wanted and unwanted markers at day 0 and 7 for conditions with isoforms of BMP. $(n=2)$ B. Scatter-plot of the mean expression (studentized values) of wanted and unwanted markers at day 0 and 7 for condition with varying cell seeding density. $(n=2)$ 
Supplementary Figure S5: Genes significantly up- or downregulated

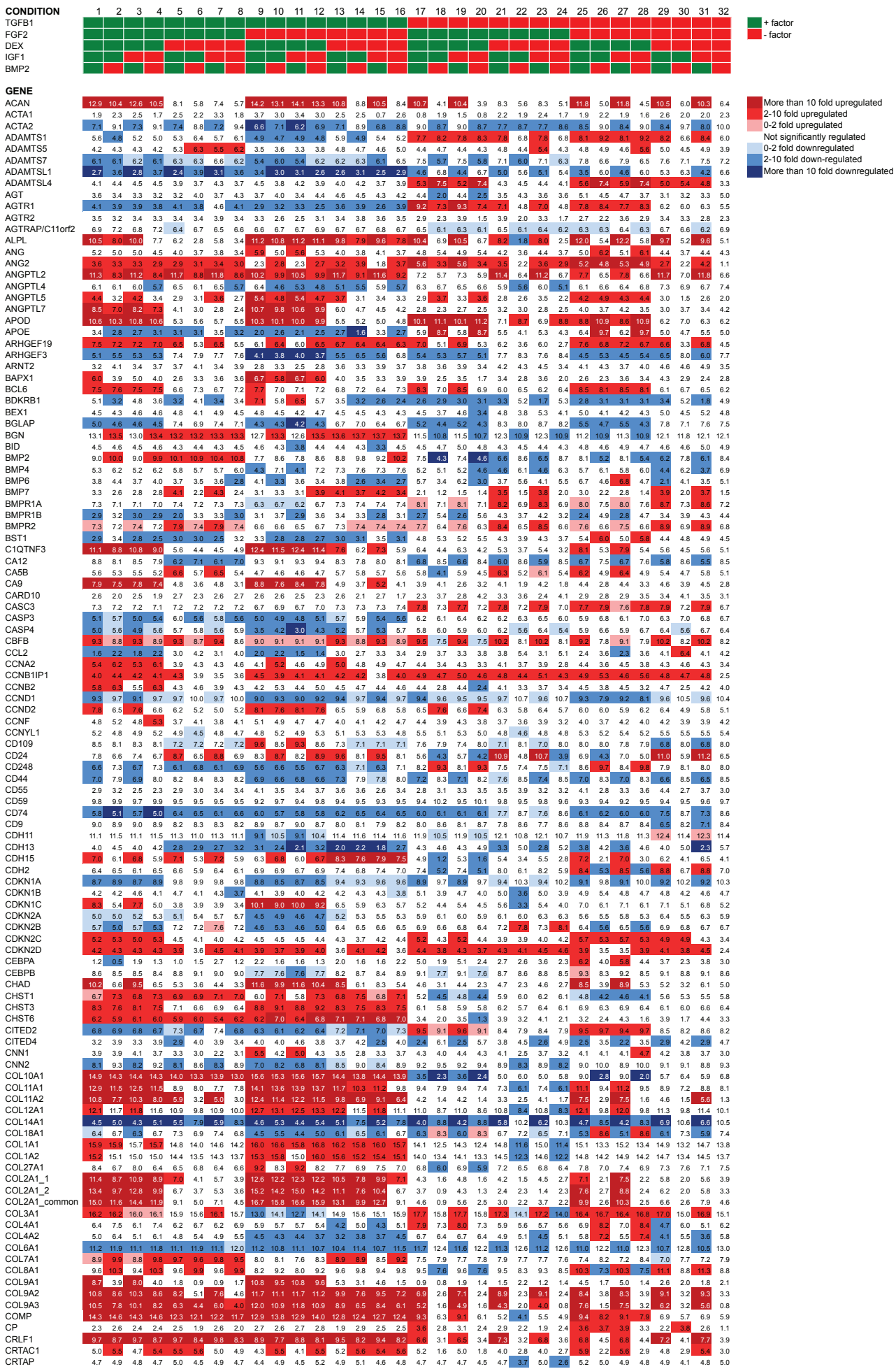




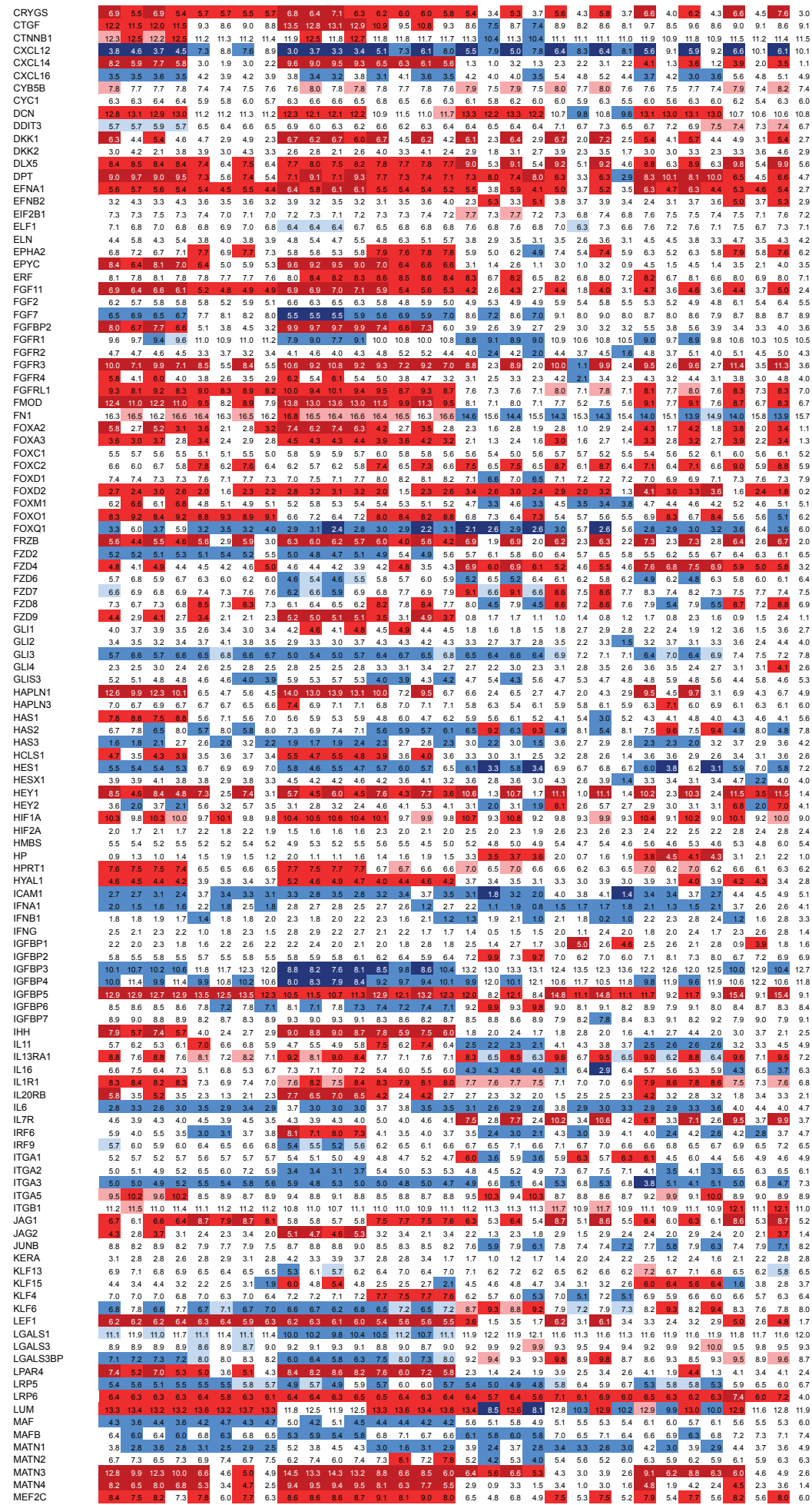




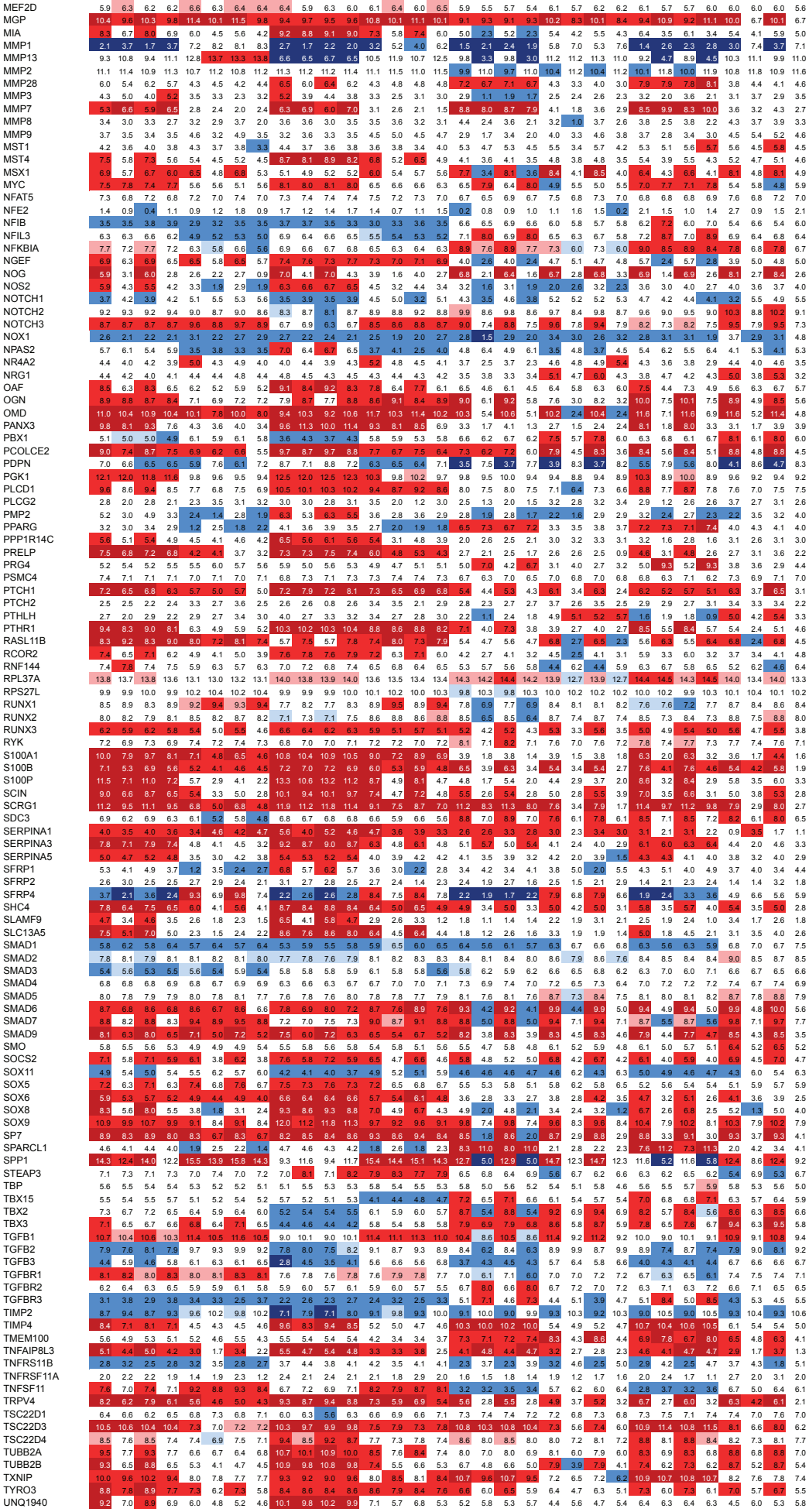




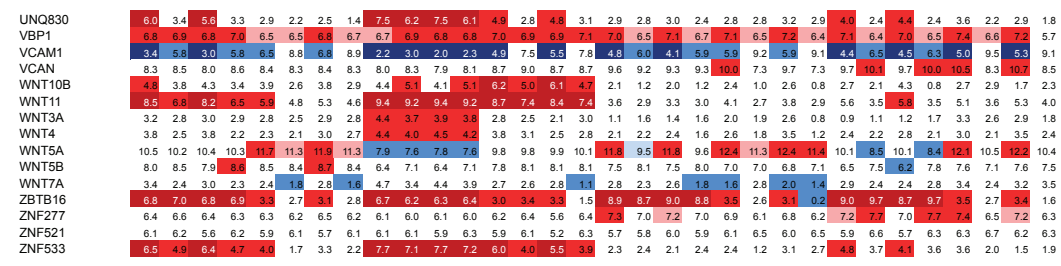

Heatmap of all genes significantly changed compared to condition 32 at day 7. Values are $\log 2$-transformed mean expressions $(n=3)$ 
Supplementary Figure S6: Genes significantly regulated between key conditions (day 7)

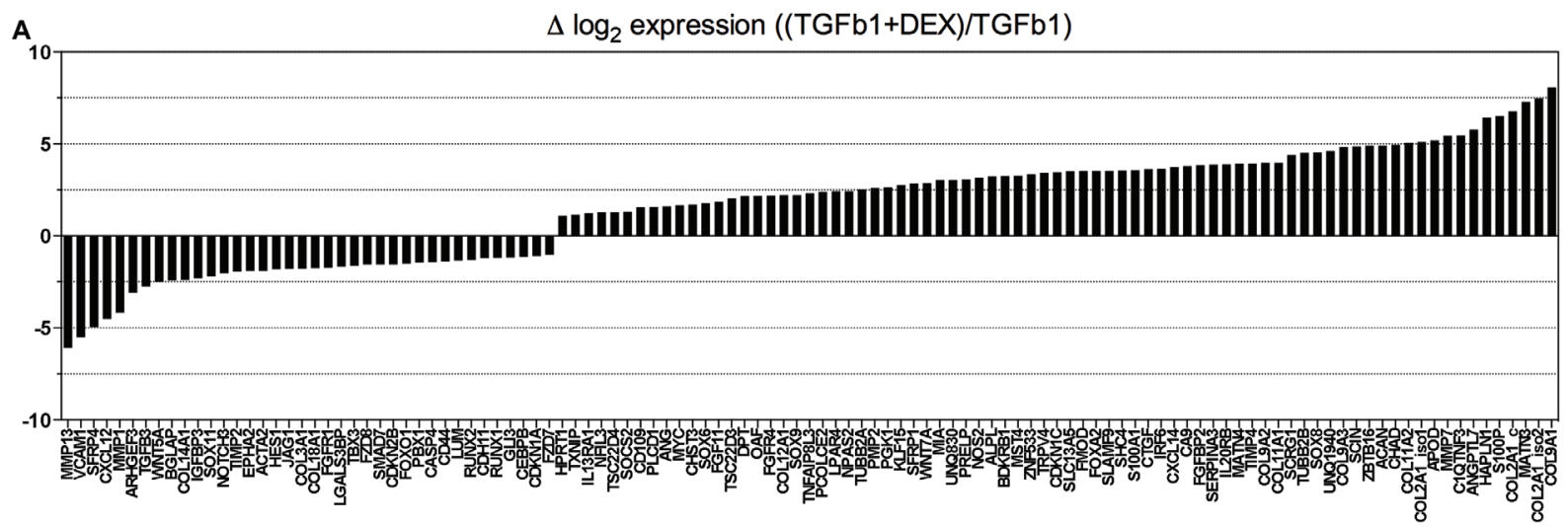

$\Delta \log _{2}$ expression ((TGFb1+BMP2+DEX)/(TGFb1+BMP2))

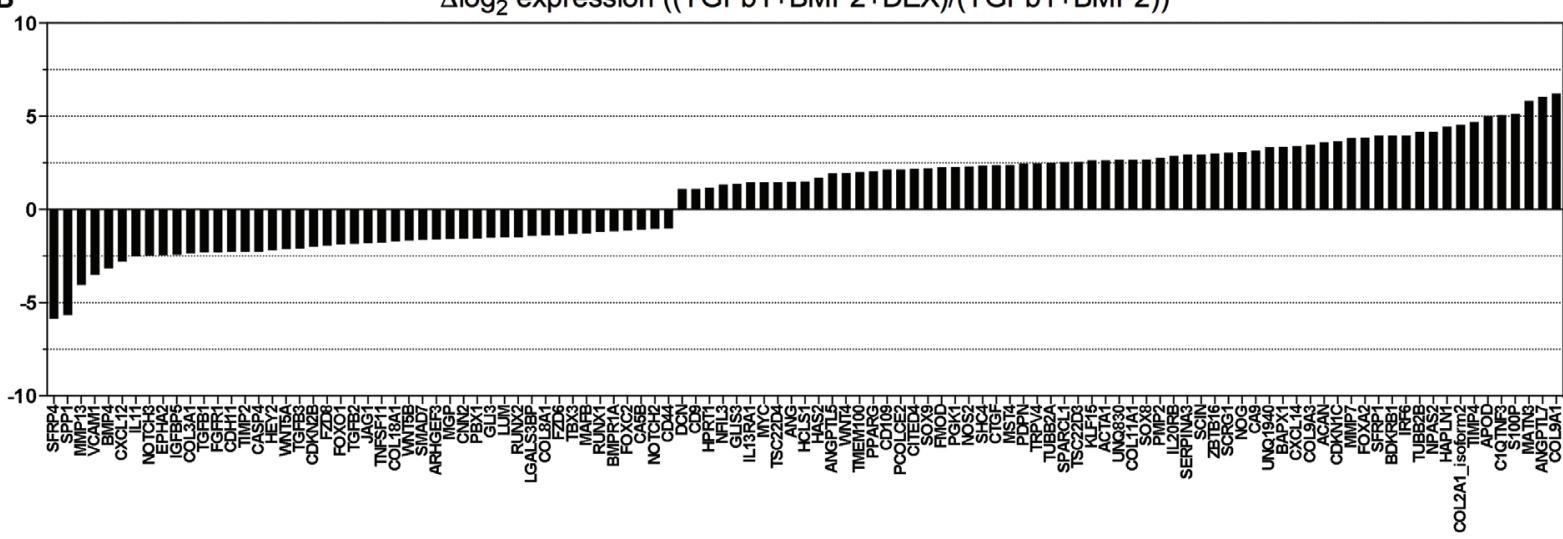

Upper panel: All significantly regulated genes ( $>2$-fold) when adding DEX to TGFB1. Lower panel: All significantly regulated genes ( $>2$-fold) when adding DEX to TGFB1+BMP2. Values represent $\log 2$ to the fold change between the gene expression in the condition without and the condition with the specified factor added. 
Supplementary Figure S7: Overlapping regulated genes when adding DEX to TGF or to TGFB1 with BMP2.

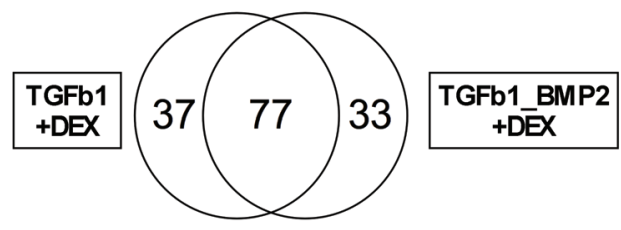

Venn diagram illustrating the overlap of genes significantly up- or downregulated corresponding to Supplementary Figure S6. 
Supplementary Table 1: Geneset of chondrogenesis related genes in the custom made multiplexed geneset.

\begin{tabular}{|c|c|c|c|c|c|}
\hline ACAN & CD59 & DKK2 & IFNB1 & MYC & SMAD2 \\
\hline ACTA1 & CD74 & DLX5 & IFNG & NFAT5 & SMAD3 \\
\hline ACTA2 & CD9 & DPT & IGFBP1 & NFE2 & SMAD4 \\
\hline ADAMTS1 & CDH11 & EFNA1 & IGFBP2 & NFIB & SMAD5 \\
\hline ADAMTS5 & CDH13 & EFNB2 & IGFBP3 & NFIL3 & SMAD6 \\
\hline ADAMTS7 & CDH15 & EIF2B1 & IGFBP4 & NFKBIA & SMAD7 \\
\hline ADAMTSL1 & $\mathrm{CDH} 2$ & ELF1 & IGFBP5 & NGEF & SMAD9 \\
\hline ADAMTSL4 & CDKN1A & ELN & IGFBP6 & NOG & SMO \\
\hline AGT & CDKN1B & EPHA2 & IGFBP7 & NOS2 & SOCS2 \\
\hline AGTR1 & CDKN1C & EPYC & IHH & NOTCH1 & SOX11 \\
\hline AGTR2 & CDKN2A & ERF & IL11 & NOTCH2 & SOX5 \\
\hline AGTRAP/C11 orf2 & CDKN2B & FGF11 & IL13RA1 & NOTCH3 & SOX6 \\
\hline ALPL & CDKN2C & FGF2 & IL16 & NOX1 & SOX8 \\
\hline ANG & CDKN2D & FGF7 & IL1R1 & NPAS2 & SOX9 \\
\hline ANG2 & CEBPA & FGFBP2 & IL20RB & NR4A2 & SP7 \\
\hline ANGPTL2 & CEBPB & FGFR1 & IL6 & NRG1 & SPARCL1 \\
\hline ANGPTL4 & CHAD & FGFR2 & IL7R & OAF & SPP1 \\
\hline ANGPTL5 & CHST1 & FGFR3 & IRF6 & OGN & STEAP3 \\
\hline ANGPTL7 & CHST3 & FGFR4 & IRF9 & OMD & TBP \\
\hline APOD & CHST6 & FGFRL1 & ITGA1 & PANX3 & TBX15 \\
\hline APOE & CITED2 & FMOD & ITGA2 & PBX1 & TBX2 \\
\hline ARHGEF19 & CITED4 & FN1 & ITGA3 & PCOLCE2 & TBX3 \\
\hline ARHGEF3 & CNN1 & FOXA2 & ITGA5 & PDPN & TGFB1 \\
\hline ARNT2 & CNN2 & FOXA3 & ITGB1 & PGK1 & TGFB2 \\
\hline BAPX1 & COL10A1 & FOXC1 & JAG1 & PLCD1 & TGFB3 \\
\hline BCL6 & COL11A1 & FOXC2 & JAG2 & PLCG2 & TGFBR1 \\
\hline BDKRB1 & COL11A2 & FOXD1 & JUNB & PMP2 & TGFBR2 \\
\hline BEX1 & COL12A1 & FOXD2 & KERA & PPARG & TGFBR3 \\
\hline BGLAP & COL14A1 & FOXM1 & KLF13 & PPP1R14C & TIMP2 \\
\hline BGN & COL18A1 & FOXO1 & KLF15 & PRELP & TIMP4 \\
\hline BID & COL1A1 & FOXQ1 & KLF4 & PRG4 & TMEM100 \\
\hline BMP2 & COL1A2 & FRZB & KLF6 & PSMC4 & TNFAIP8L3 \\
\hline BMP4 & COL27A1 & FZD2 & LEF1 & PTCH1 & TNFRS11B \\
\hline BMP6 & COL2A1_common & FZD4 & LGALS1 & РTCH2 & TNFRSF11A \\
\hline BMP7 & COL2A1_isoform 1 & FZD6 & LGALS3 & PTHLH & TNFSF11 \\
\hline BMPR1A & COL2A1_isoform2 & FZD7 & LGALS3BP & PTHR1 & TRPV4 \\
\hline BMPR1B & COL $3 \mathrm{~A} 1{ }^{-}$ & FZD8 & LPAR4 & RASL11B & TSC22D1 \\
\hline BMPR2 & COL4A1 & FZD9 & LRP5 & RCOR2 & TSC22D3 \\
\hline BST1 & COL4A2 & GLI1 & LRP6 & RNF144 & TSC22D4 \\
\hline C1QTNF3 & COL6A1 & GLI2 & LUM & RPL37A & TUBB2A \\
\hline CA12 & COL7A1 & GLI3 & MAF & RPS27L & TUBB2B \\
\hline CA5B & COL8A1 & GLI4 & MAFB & RUNX1 & TXNIP \\
\hline CA9 & COL9A1 & GLIS3 & MATN1 & RUNX2 & TYRO3 \\
\hline CARD10 & COL9A2 & HAPLN1 & MATN2 & RUNX3 & UNQ1940 \\
\hline CASC3 & COL9A3 & HAPLN3 & MATN3 & RYK & UNQ830 \\
\hline CASP3 & COMP & HAS1 & MATN4 & S100A1 & VBP1 \\
\hline CASP4 & $\mathrm{CP}$ & HAS2 & MEF2C & S100B & VCAM1 \\
\hline CBFB & CRLF1 & HAS3 & MEF2D & S100P & VCAN \\
\hline CCL2 & CRTAC1 & HCLS1 & MGP & SCIN & WNT10B \\
\hline CCNA2 & CRTAP & HES1 & MIA & SCRG1 & WNT11 \\
\hline CCNB1IP1 & CRYGS & HESX1 & MMP1 & SDC3 & WNT3A \\
\hline CCNB2 & CTGF & HEY1 & MMP13 & SERPINA1 & WNT4 \\
\hline CCND1 & CTNNB1 & HEY2 & MMP2 & SERPINA3 & WNT5A \\
\hline CCND2 & CXCL12 & HIF1A & MMP28 & SERPINA5 & WNT5B \\
\hline CCNF & CXCL14 & HIF2A & MMP3 & SFRP1 & WNT7A \\
\hline CCNYL1 & CXCL16 & HMBS & MMP7 & SFRP2 & ZBTB16 \\
\hline CD109 & CYB5B & HP & MMP8 & SFRP4 & ZNF277 \\
\hline CD24 & CYC1 & HPRT1 & MMP9 & SHC4 & ZNF521 \\
\hline CD248 & $\mathrm{DCN}$ & HYAL1 & MST1 & SLAMF9 & ZNF533 \\
\hline CD44 & DDIT3 & ICAM1 & MST4 & SLC13A5 & \\
\hline CD55 & DKK1 & IFNA1 & MSX1 & SMAD1 & \\
\hline
\end{tabular}

COL2A1_common recoqnizes both splice variants of COL2A1, COL2A1_isoform 1 recoqnizes variant 1 (fetal type) and COL2A1_isoform 2 recoqnizes variant 2 (mature type). Genes in BOLD were included as endogenous referencegenes. 
Supplementary Table S2: Statistical response analysis of main effects and second and third order interactions at day 1 and 7.

Factorial Fit Of Wanted Genes versus All Five Factors Including Two-Factor Interactions on Day 7

\begin{tabular}{lrrrl}
\hline \multicolumn{1}{c}{ Term } & \multicolumn{1}{c}{ Effect } & \multicolumn{1}{c}{ Coef } & \multicolumn{1}{c}{ T } & \multicolumn{1}{l}{ P } \\
\hline Constant & & 9.3892 & 110.81 & $\mathbf{0 . 0 0 0}$ \\
TGFb1 & 3.3493 & 1.6746 & 19.76 & $\mathbf{0 . 0 0 0}$ \\
FGF2 & -1.3188 & -0.6594 & -7.78 & $\mathbf{0 . 0 0 0}$ \\
DEX & 1.9587 & 0.9794 & 11.56 & $\mathbf{0 . 0 0 0}$ \\
IGF1 & 0.0902 & 0.0451 & 0.53 & 0.596 \\
BMP2 & 1.5581 & 0.7791 & 9.19 & $\mathbf{0 . 0 0 0}$ \\
TGFb1*FGF2 & -0.3273 & -0.1637 & -1.93 & 0.057 \\
TGFb1*DEX & 1.1082 & 0.5541 & 6.54 & $\mathbf{0 . 0 0 0}$ \\
TGFb1*IGF1 & 0.0979 & 0.0489 & 0.58 & 0.565 \\
TGFb1*BMP2 & -0.4874 & -0.2437 & -2.88 & $\mathbf{0 . 0 0 5}$ \\
FGF2*DEX & 0.0864 & 0.0432 & 0.51 & 0.612 \\
FGF2*IGF1 & 0.0052 & 0.0026 & 0.03 & 0.976 \\
FGF2*BMP2 & -0.1035 & -0.0517 & -0.61 & 0.543 \\
DEX*IGF1 & -0.1022 & -0.0511 & -0.6 & 0.548 \\
DEX*BMP2 & 0.2296 & 0.1148 & 1.35 & 0.180 \\
IGF1*BMP2 & 0.0462 & 0.0231 & 0.27 & 0.786 \\
TGFb1*DEX*BMP2 & -0.3821 & -0.1911 & -2.25 & $\mathbf{0 . 0 2 7}$ \\
\hline R ${ }^{2}=91.32 \%$ & & & & \\
\hline
\end{tabular}

Factorial Fit Of Unwanted Genes versus All Five Factors Including Two-Factor Interactions on Day 7

\begin{tabular}{lrrrl}
\hline \multicolumn{1}{c}{ Term } & Effect & \multicolumn{1}{c}{ Coef } & \multicolumn{1}{c}{ T } & P \\
\hline Constant & & 10.8056 & 225.62 & 0.000 \\
TGFb1 & 1.0835 & 0.5418 & 11.31 & 0.000 \\
FGF2 & -0.2662 & -0.1331 & -2.78 & 0.007 \\
DEX & 0.1495 & 0.0747 & 1.56 & 0.123 \\
IGF1 & -0.0217 & -0.0109 & -0.23 & 0.821 \\
BMP2 & 1.0365 & 0.5183 & 10.82 & 0.000 \\
TGFb1*FGF2 & 0.0332 & 0.0166 & 0.35 & 0.730 \\
TGFb1*DEX & -0.1856 & -0.0928 & -1.94 & 0.057 \\
TGFb1*IGF1 & 0.0361 & 0.018 & 0.38 & 0.707 \\
TGFb1*BMP2 & -0.9729 & -0.4865 & -10.16 & 0.000 \\
FGF2*DEX & 0.2545 & 0.1272 & 2.66 & 0.010 \\
FGF2*IGF1 & -0.0015 & -0.0007 & -0.02 & 0.988 \\
FGF2*BMP2 & 0.148 & 0.074 & 1.54 & 0.127 \\
DEX*IGF1 & 0.0188 & 0.0094 & 0.2 & 0.845 \\
DEX*BMP2 & -0.059 & -0.0295 & -0.62 & 0.540 \\
IGF1*BMP2 & 0.0155 & 0.0078 & 0.16 & 0.872 \\
TGFb1*FGF2*DEX & 0.2719 & 0.1359 & 2.84 & $\mathbf{0 . 0 0 6}$ \\
\hline R $^{2}=84.67 \%$ & \multicolumn{3}{c}{} \\
\hline
\end{tabular}


Factorial Fit Of Wanted Genes versus All Five Factors Including Two-Factor Interactions on Day 1

\begin{tabular}{lrrrc}
\hline \multicolumn{1}{c}{ Term } & Effect & \multicolumn{1}{l}{ Coef } & \multicolumn{1}{c}{ T } & \multicolumn{1}{c}{ P } \\
\hline Constant & & 7.5912 & 218.76 & $\mathbf{0 . 0 0 0}$ \\
TGFb1 & 0.5807 & 0.2904 & 8.37 & $\mathbf{0 . 0 0 0}$ \\
FGF2 & -0.4061 & -0.2031 & -5.85 & $\mathbf{0 . 0 0 0}$ \\
DEX & 0.4144 & 0.2072 & 5.97 & $\mathbf{0 . 0 0 0}$ \\
IGF1 & -0.0429 & -0.0214 & -0.62 & 0.540 \\
BMP2 & 0.735 & 0.3675 & 10.59 & $\mathbf{0 . 0 0 0}$ \\
TGFb1*FGF2 & -0.0912 & -0.0456 & -1.31 & 0.196 \\
TGFb1*DEX & 0.1099 & 0.0549 & 1.58 & 0.122 \\
TGFb1*IGF1 & -0.0528 & -0.0264 & -0.76 & 0.452 \\
TGFb1*BMP2 & -0.3338 & -0.1669 & -4.81 & $\mathbf{0 . 0 0 0}$ \\
FGF2*DEX & -0.0326 & -0.0163 & -0.47 & 0.641 \\
FGF2*IGF1 & -0.022 & -0.011 & -0.32 & 0.753 \\
FGF2*BMP2 & -0.1376 & -0.0688 & -1.98 & 0.055 \\
DEX*IGF1 & -0.0121 & -0.0061 & -0.17 & 0.862 \\
DEX*BMP2 & 0.0698 & 0.0349 & 1.01 & 0.321 \\
IGF1*BMP2 & 0.0409 & 0.0205 & 0.59 & 0.559 \\
\hline R $^{2}=88.38 \%$ & & & & \\
\hline
\end{tabular}

Factorial Fit Of Unwanted Genes versus All Five Factors Including Two-Factor Interactions on Day 1

\begin{tabular}{lrrrc}
\hline \multicolumn{1}{c}{ Term } & Effect & \multicolumn{1}{c}{ Coef } & \multicolumn{1}{c}{ T } & \multicolumn{1}{c}{ P } \\
\hline Constant & & 9.5693 & 337.77 & $\mathbf{0 . 0 0 0}$ \\
TGFb1 & 0.1489 & 0.0745 & 2.63 & $\mathbf{0 . 0 1 2}$ \\
FGF2 & -0.2385 & -0.1193 & -4.21 & $\mathbf{0 . 0 0 0}$ \\
DEX & -0.0565 & -0.0283 & -1 & 0.325 \\
IGF1 & -0.0138 & -0.0069 & -0.24 & 0.808 \\
BMP2 & 0.2797 & 0.1398 & 4.94 & $\mathbf{0 . 0 0 0}$ \\
TGFb1*FGF2 & -0.0746 & -0.0373 & -1.32 & 0.196 \\
TGFb1*DEX & -0.0899 & -0.0449 & -1.59 & 0.121 \\
TGFb1*IGF1 & 0.0007 & 0.0004 & 0.01 & 0.990 \\
TGFb1*BMP2 & -0.1963 & -0.0981 & -3.46 & $\mathbf{0 . 0 0 1}$ \\
FGF2*DEX & 0.0284 & 0.0142 & 0.5 & 0.619 \\
FGF2*IGF1 & 0.0123 & 0.0061 & 0.22 & 0.830 \\
FGF2*BMP2 & -0.0437 & -0.0218 & -0.77 & 0.445 \\
DEX*IGF1 & -0.0155 & -0.0078 & -0.27 & 0.786 \\
DEX*BMP2 & -0.0321 & -0.0161 & -0.57 & 0.574 \\
IGF1*BMP2 & 0.0536 & 0.0268 & 0.95 & 0.350 \\
\hline $\mathrm{R}^{2}=65.67 \%$ & & & & \\
\hline
\end{tabular}

$\mathrm{R}^{2}$ represent the proportion of variation in the response data that is explained by the fitted mathematical model. 
Supplementary Table S3: List of significantly regulated genes uniformly up- or downregulated by DEX in the presence of TGFb1 alone or in combination with BMP2 corresponding to the overlap in the Venn-diagram in Supplementary Figure S6.

\begin{tabular}{ll|llll} 
Down & & $\mathbf{U p}$ & & & \\
\hline ARHGEF3 & LGALS3BP & ACAN & CXCL14 & NOS2 & TRPV4 \\
CASP4 & LUM & ANG & FMOD & NPAS2 & TSC22D3 \\
CD44 & MMP13 & ANGPTL7 & FOXA2 & PCOLCE2 & TSC22D4 \\
CDH11 & NOTCH3 & APOD & HAPLN1 & PGK1 & TUBB2A \\
CDKN2B & PBX1 & BDKRB1 & HPRT1 & PMP2 & TUBB2B \\
COL18A1 & RUNX1 & C1QTNF3 & IL13RA1 & S100P & UNQ1940 \\
COL3A1 & RUNX2 & CA9 & IL20RB & SCIN & UNQ830 \\
CXCL12 & SFRP4 & CD109 & IRF6 & SCRG1 & ZBTB16 \\
EPHA2 & SMAD7 & CDKN1C & KLF15 & SERPINA3 & \\
FGFR1 & TBX3 & COL11A1 & MATN3 & SFRP1 & \\
FOXO1 & TGFB3 & COL2A1_iso2 & MMP7 & SHC4 & \\
FZD8 & TIMP2 & COL9A1 & MST4 & SOX8 & \\
GLI3 & VCAM1 & COL9A3 & MYC & SOX9 & \\
JAG1 & WNT5A & CTGF & NFIL3 & TIMP4 & \\
\hline
\end{tabular}


Supplementary Table S4: List of primary and secondary antibodies used for immunohistochemistry.

\begin{tabular}{|c|c|c|c|c|}
\hline $\begin{array}{l}\text { Antibodies for } \\
\text { immunohistochemistry }\end{array}$ & $\begin{array}{l}\text { Working } \\
\text { concentration }\end{array}$ & Protein & Company & Catalog number \\
\hline $\begin{array}{l}\text { Monoclonal Mouse IgG1 } \\
\text { anti-COL2 }\end{array}$ & $0.83 \mathrm{mg} / \mathrm{mL}$ & Type 2 collagen & $\begin{array}{l}\text { MP } \\
\text { Biomedicals }\end{array}$ & $\begin{array}{l}\text { Clone II-4C11/ } \\
\# 863171\end{array}$ \\
\hline $\begin{array}{l}\text { Monoclonal Mouse } \\
\text { IgG1 anti-ACAN }\end{array}$ & $4.55 \mathrm{mg} / \mathrm{mL}$ & Aggrecan & Biosource & $\begin{array}{l}\text { Clone } \\
\text { 969D4D11/ } \\
58.146 .21\end{array}$ \\
\hline $\begin{array}{l}\text { Monoclonal Mouse IgG2a } \\
\text { anti-COL1 }\end{array}$ & $1 \mathrm{mg} / \mathrm{mL}$ & Type I collagen & $\begin{array}{l}\text { MP } \\
\text { Biomedicals }\end{array}$ & $\begin{array}{l}\text { Clone I-8H5 / } \\
\# 863170\end{array}$ \\
\hline $\begin{array}{l}\text { Polyclonal Rabbit anti- } \\
\text { SOX9 }\end{array}$ & $0.2 \mathrm{mg} / \mathrm{mL}$ & $\begin{array}{l}\text { SRY-box } \\
\text { containing gene } 9\end{array}$ & Millipore & AB5535 \\
\hline $\begin{array}{l}\text { Alexa488-conjugated goat } \\
\text { anti-rabbit }\end{array}$ & $5 \mathrm{mg} / \mathrm{mL}$ & & Invitrogen & \\
\hline $\begin{array}{l}\text { Cy3-conjugated donkey } \\
\text { anti-mouse IgG }\end{array}$ & $2 \mathrm{mg} / \mathrm{mL}$ & & $\begin{array}{l}\text { Jackson } \\
\text { Immuno } \\
\text { Research }\end{array}$ & \\
\hline
\end{tabular}

